\title{
Microfluidics: Fluid physics at the nanoliter scale
}

\author{
Todd M. Squires* \\ Departments of Physics and Applied \& Computational Mathematics, California Institute of \\ Technology, Pasadena, California 91125, USA \\ Stephen R. Quake ${ }^{\dagger}$ \\ Departments of Applied Physics and Physics, California Institute of Technology, \\ Pasadena, California 91125, USA
}

(Published 6 October 2005)

\begin{abstract}
Microfabricated integrated circuits revolutionized computation by vastly reducing the space, labor, and time required for calculations. Microfluidic systems hold similar promise for the large-scale automation of chemistry and biology, suggesting the possibility of numerous experiments performed rapidly and in parallel, while consuming little reagent. While it is too early to tell whether such a vision will be realized, significant progress has been achieved, and various applications of significant scientific and practical interest have been developed. Here a review of the physics of small volumes (nanoliters) of fluids is presented, as parametrized by a series of dimensionless numbers expressing the relative importance of various physical phenomena. Specifically, this review explores the Reynolds number $\mathrm{Re}$, addressing inertial effects; the Péclet number Pe, which concerns convective and diffusive transport; the capillary number Ca expressing the importance of interfacial tension; the Deborah, Weissenberg, and elasticity numbers $\mathrm{De}, \mathrm{Wi}$, and $\mathrm{El}$, describing elastic effects due to deformable microstructural elements like polymers; the Grashof and Rayleigh numbers $\mathrm{Gr}$ and Ra, describing density-driven flows; and the Knudsen number, describing the importance of noncontinuum molecular effects. Furthermore, the long-range nature of viscous flows and the small device dimensions inherent in microfluidics mean that the influence of boundaries is typically significant. A variety of strategies have been developed to manipulate fluids by exploiting boundary effects; among these are electrokinetic effects, acoustic streaming, and fluid-structure interactions. The goal is to describe the physics behind the rich variety of fluid phenomena occurring on the nanoliter scale using simple scaling arguments, with the hopes of developing an intuitive sense for this occasionally counterintuitive world.
\end{abstract}

\section{CONTENTS}

I. Introduction

978

II. Dimensionless Numbers in Microfluidics

A. The Reynolds number: Inertial irrelevance

B. The Péclet number and the problem of mixing

1. Sensing, filtering, and fabricating with parallel laminar flows

a. T sensor

983

b. Filtration without membranes

983

c. Fabricating and interrogating using multiple laminar streams

984

2. Fighting diffusion: Particle separations

3. Beating diffusion: When mixing matters

a. Taylor dispersion

b. Rotary mixer

c. Mixing by chaotic advection

985

985

986

987

988

C. The capillary number: Free-surface deformations

1. Droplet formation in two-phase flows

*Present address: Department of Chemical Engineering, University of California, Santa Barbara, CA 93106. Electronic address: squires@engineering.ucsb.edu

†Present address: Department of Bioengineering, Stanford University, Stanford, CA 94305. Electronic address: quake@stanford.edu

2. Fluid control using patterned surfaces 990

3. Fluid manipulation with capillary forces 991

a. Solid-liquid interfacial energy manipulation 991

b. Surfaces with wettability gradients 992

i. Thermal gradients [Fig. 19(a)] 992

ii. Reactive fluids [Fig. 19(b)] 992

iii. Self-propelling liquid bi-slugs [Fig. 19(c)] 992

iv. Optowetting [Fig. 19(d)] 992

v. Electrowetting 993

vi. Dielectrophoresis 994

c. Liquid-liquid surface tension manipulation 994

D. The Weissenberg and Deborah numbers: Let the mountains flow

1. Microfluidic single-polymer studies

2. Elastic instabilities

3. Controlled polymer deformation for separations

998

E. The Grashof and Rayleigh numbers: Tracer-driven flows

1. Protein crystallization

1001

1002

2. Polymerase chain reaction (PCR) 1004

3. Electrohydrodynamic instabilities 1004

F. The Knudsen number: When molecules matter 1005

1. Molecular effects in gases 1005

2. Fluid slip over nonwetting surfaces 1006

III. Driving Flows at the Boundaries 1007

A. Electrokinetic effects 1007

1. Electrostatics in ionic solutions 1007 
2. Electrokinetics: Electro-osmosis and electrophoresis

3. Recent variations on classical electrokinetic techniques

a. Patterned surfaces with nonuniform charge

b. Active control of surface charge

c. Induced-charge electrokinetic phenomena

B. Acoustic streaming

1. Quartz wind

2. Boundary-induced streaming

3. Cavitation microstreaming

C. Fluid-structure interactions

1. Driving flows with soft walls

2. Intelligent walls made of responsive hydrogel

Acknowledgments

List of Symbols

References

\section{INTRODUCTION}

The integrated circuit revolution has allowed largescale automation of computation, fundamentally altering the way we live and do science. A natural question to ask is whether it is possible to automate biology and chemistry to a similar extent, and if so, would the consequences be as dramatic? The industrial approach to addressing biological integration has come in the form of enormous robotic fluidic workstations that take up entire laboratories and require considerable expense, space, and labor. This approach is reminiscent of the macroscopic approach to circuits consisting of massive vacuum-tube-based arrays in the early twentieth century. Like the vacuum-tube computing revolution, such biological automation has already been highly productive and has led to whole genome sequencing and analysis - a new revolution in biology. Microfluidics, which is essentially a field dedicated to miniaturized plumbing and fluidic manipulation, offers the possibility of solving outstanding system integration issues for biology and chemistry by allowing automation to proceed to scales that will rival electronic integrated circuits (Fig. $1)$.

Although most current effort in microfluidics concerns devices with applications in chemistry, biology, and medicine, there are also applications in the physical sciences for control systems and heat management [e.g., Zhang et al. (2002)], energy generation [e.g., Ferrigno et al. (2002) and Choban et al. (2004)], and display technology [e.g., Hayes and Feenstra (2003)]. There is a long history of using fluidics as control systems, ranging from logic devices to thrust reversers in aircraft; this program ultimately foundered in part because scaling properties of the fluid physics prevented miniaturization (Humphrey and Tarumoto, 1965; Foster and Parker, 1970; Joyce, 1983). Liquid crystal displays and ink jet printers [e.g., Bassous et al. (1977)] are ubiquitous consumer products that can be thought of as microfluidic devices, and have had enormous industrial impact. As fuel cells become more widely deployed, it is likely that they will

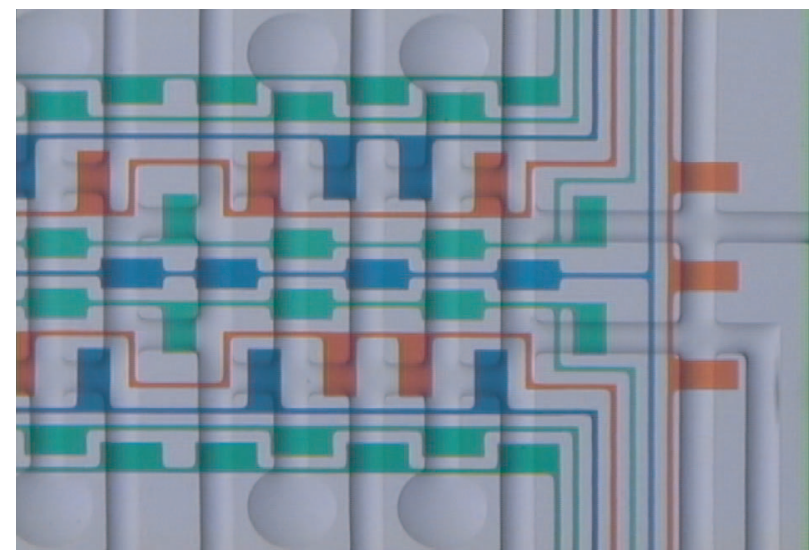

FIG. 1. (Color) Sophisticated networks of fluidic microchannels allow for unprecedented automation and parallelization of biological and chemical systems. Pictured is a large-scale, integrated chip to measure protein interactions. The colored control lines manipulate valves that function as gates and pumps, which drive reagent solutions through the uncolored channels to be stored in circular chambers for subsequent reaction. Circular chambers are $250 \mu \mathrm{m}$ diameter. (Figure courtesy of S. Maerkl.)

ultimately incorporate some sort of microfluidic plumbing.

One important manner in which microfluidics differs from microelectronics is that the fundamental physics changes more rapidly as the size scale is decreased. Despite decades of ever smaller transistors and higher densities in semiconductor electronics, the industry has yet to reach the length scale where scaling has caused a qualitative change in physical phenomena, e.g., from the classical to the quantum regime. Single electron transistors and other mesoscopic devices have been developed, yet remain exotic objects of laboratory exploration. By contrast, fluidic systems can rather quickly reach length scales where the fundamental fluid physics changes dramatically. One of the best-known examples is that mass transport in microfluidic devices is generally dominated by viscous dissipation, and inertial effects are generally negligible. Since inertia provides the nonlinearity that is responsible for numerous instabilities and for turbulence itself, its loss might seem to render microfluidic flows uninteresting.

In fact, we argue that the opposite is true. Microfluidic physics is quite rich, drawing from much of classical physics and chemistry-fluid mechanics, electrostatics, thermodynamics, statistical mechanics, elasticity, and polymer physics, to name a few. Notably, the small dimensions that quell the inertial nonlinearity bring other physical phenomena, less familiar on our macroscale, to prominence.

Following the invention of the transistor, some concerned themselves primarily with designing and building circuits to perform various computational tasks, whereas others continued to study and explore the science underlying semiconductors and microcircuits. Similarly in microfluidics some focus primarily on developing microfluidic devices and tools during which, of course, scientific 
issues arise and are explored, whereas others focus on exploring and understanding the physics of these systems.

In this spirit, the focus of this review lies explicitly with the physics underlying microfluidic devices, emphasizing the variety of physical phenomena and the manner in which they have been exploited. Perspective pieces, both early (Manz et al., 1993; Ramsey et al., 1995) and more recent (Ramsey, 1999; Figeys and Pinto, 2000; Stone and Kim, 2001; Meldrum and Holl, 2002; Hong and Quake, 2003), describe the promise, progress, and challenges for the field. "Lab on a chip" or "micro total analysis systems" (Manz et al., 1993) have recently been reviewed in a pair of companion articles, one focusing on the history and overall approach (Reyes et al., 2002), and the other on standard operations in analytical chemistry and their microfluidic applications (Auroux et al., 2002). Reviews of more specific applications for microfluidics include protein crystallization (Hansen and Quake, 2003), proteomics (Dolnik, 1997, 1999; Dolnik and Hutterer, 2001; Figeys and Pinto, 2001; Hancock et al., 2002; Lion et al., 2003), microfluidics based on micromechanical systems (MEMS) (Ho and Tai, 1998; Verpoorte and De Rooij, 2003), micropumps (Nguyen et al., 2002; Laser and Santiago, 2004), clinical and forensic applications (Thormann et al., 2001; Verpoorte, 2002), sample pretreatment (Lichtenberg et al., 2002), and molecular diagnostics (Landers, 2003; Auroux et al., 2004). The advantages and applications of soft (rather than silicon) microfluidics have been reviewed (McDonald et al., 2000; Quake and Scherer, 2000; Whitesides and Stroock, 2001; Whitesides et al., 2001; Ng et al., 2002; Sia and Whitesides, 2003). Finally, separation techniques, particularly for nucleic acids, occupy a central role in many microfluidic devices. Specific reviews include DNA separation and analysis methods in the microfluidic context (Ugaz et al., 2004), DNA separation in microfabricated devices (Tegenfeldt et al., 2004), the theory of polymer separation (Viovy, 2000; Slater et al., 2002, 2003; Ashton et al., 2003; Sartori et al., 2003), and microchip-based polymer electrophoresis (Bousse et al., 2000; Bruin, 2000; Dolnik et al., 2000).

Rather than duplicate these efforts, the present review emphasizes the physics of the field over its specific applications, and should complement other physically minded reviews (Brody et al., 1996; Beebe et al., 2002; Stone et al., 2004). Specifically, we consider fluid physics that occurs within channels with typically $\sim 100 \mu \mathrm{m}$ transverse dimension-for reference, $1 \mathrm{nl}=(100 \mu \mathrm{m})^{3}$. We note, however, that the physics explored here is also relevant for complex fluids and colloids, porous media, and biological systems, to name a few.

The essential fluid physics of a system is dictated by a competition between various phenomena, which is captured by a series of dimensionless numbers expressing their relative importance. These dimensionless numbers form a sort of parameter space for microfluidic physics, which we set out to explore. We organized this review around those numbers-in some cases, they scale with a characteristic system length such as channel size and thus indicate an important change in physics going from everyday fluid volumes to the nanoliter scale. In other cases, the numbers are independent of length scale yet still have important implications and applications.

We begin our review of each number with an intuitive, paradigmatic model example to illustrate the basic physics at hand with simple scaling arguments. Following each model example, we discuss various devices and systems that have been designed to utilize or probe the physics at hand. In the latter stages of the review we consider another set of phenomena that are characteristic of microfluidic devices-the importance of boundary conditions, and in particular the dominant role played by surface effects. Such boundary-driven effects are of particular importance to microfludic devices because the characteristic geometries of microfluidic devices nearly always have large surface-area-to-volume ratios. Specifically, we discuss electrokinetic effects, acoustic streaming, and fluid-structure interactions.

The past decade has seen a tremendous effort in microfluidic research, with many thousands of research articles and several dedicated journals. In reviewing the literature, we have sought to include references from the archival scientific literature which are, to the best of our knowledge, first chronologically or which possess significant pedagogical value with respect to the physics involved. There are other useful references which in some cases predate the archival publications to be found in various yearly conference proceedings such as MicroTAS and Hilton Head Sensors and Actuators which are not indexed in the Web of Science; for reasons of scope and accessibility they are not included in this review.

Microfluidics is an enormous field, and we have been ambitious in terms of the scope of physical phenomena treated here. Many, even most, of these phenomena could merit their own review. We have inevitably overlooked interesting, important, and deserving work in preparing this review, and we apologize in advance for such oversights. This field is as interdisciplinary as any, and the numerous disciplines involved have and will continue to overlook and reinvent each others' work. Our hope in this review is to help researchers think intuitively about the diverse physics of these systems, to attempt to bridge the various communities involved, and to convey the interest, elegance, and variety of physical phenomena that manifest themselves on the nanoliter scale.

\section{DIMENSIONLESS NUMBERS IN MICROFLUIDICS}

A wide variety of physical phenomena occur in microfluidic devices, the importance of which must be judged against competing phenomena. Dimensionless numbers expressing the ratio of these phenomena give a sense for where a system sits in fluidic parameter space. Motivated by this picture, we review this parameter space, one dimension at a time, by exploring the dimensionless numbers listed in Table I: the Reynolds number Re, relating inertial forces to viscous forces; the Péclet number 
TABLE I. Dimensionless numbers used in this review.

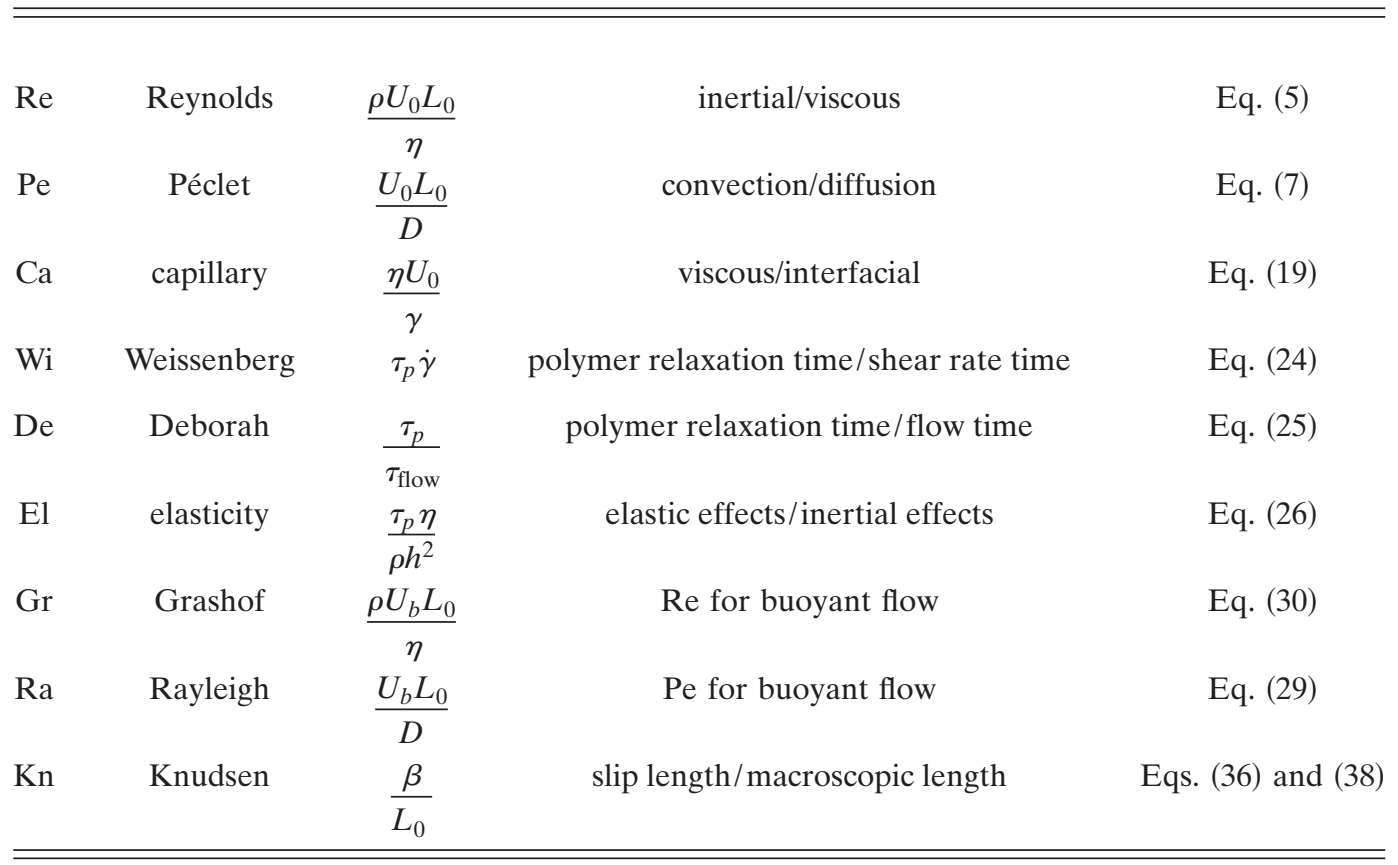

Pe, relating convection to diffusion; the capillary number $\mathrm{Ca}$, relating viscous forces to surface tension; the Deborah, Weissenberg, and elasticity numbers De, Wi, and El, expressing elastic effects; the Grashof and Rayleigh numbers $\mathrm{Gr}$ and $\mathrm{Ra}$, relating transport mechanisms in buoyancy-driven flows; and the Knudsen number $\mathrm{Kn}$, relating microscopic to macroscopic length scales. Throughout this review, we will make reference to the standard microchannels shown in Fig. 2.

Before beginning, however, we very briefly review basic fluid properties and the dimensional parameters that characterize them. Fluids are continuum materials, and so discrete quantities like mass and force give way to continuous fields like density $\rho$ and force density $f$ that are defined per unit volume. The concept of a small fluid element is often invoked by analogy with discrete mechanics. Forces on such elements arise from fluid stresses $\overleftrightarrow{\boldsymbol{\sigma}}$ (forces per unit area) exerted on the element surfaces, in addition to externally applied body forces $\mathbf{f}$ exerted on the bulk of the element.

The velocity field for a Newtonian fluid obeys the Navier-Stokes equations, which essentially represent the

(a)
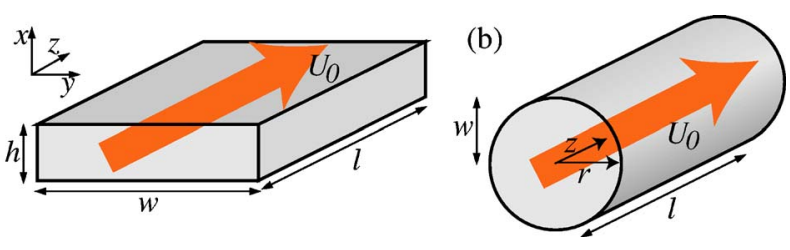

FIG. 2. (Color in online edition) Model (a) rectangular and (b) circular microchannels, through which fluid flows with characteristic velocity scale $U_{0}$. Channel length will be denoted $l$, width (or radius) $w$, and height (shortest dimension) $h$. The coordinate $z$ points downstream, $y$ spans the width, and $x$ spans the height. continuum version of $\mathbf{F}=m \mathbf{a}$ on a per unit volume basis:

$$
\rho\left(\frac{\partial \mathbf{u}}{\partial t}+\mathbf{u} \cdot \nabla \mathbf{u}\right)=\nabla \cdot \overleftrightarrow{\boldsymbol{\sigma}}+\mathbf{f}=-\nabla p+\eta \nabla^{2} \mathbf{u}+\mathbf{f}
$$

where inertial acceleration terms appear on the left and forces on the right. Here $\mathbf{f}$ represents body force densities, which will include many of the effects discussed in this review. When inertial forces are small compared to viscous forces, which is usually the case in microfluidic devices, the nonlinear term can be neglected, leaving the Stokes equation

$$
\rho \frac{\partial \mathbf{u}}{\partial t}=\nabla \cdot \boldsymbol{\sigma}+\mathbf{f}=-\nabla p+\eta \nabla^{2} \mathbf{u}+\mathbf{f}
$$

In both cases, mass conservation requires

$$
\frac{\partial \rho}{\partial t}+\nabla \cdot(\rho \mathbf{u})=0
$$

giving the incompressibility condition $\nabla \cdot \mathbf{u}=0$ for slowly flowing fluids with nearly constant density like water.

Physically, the tensor stress $\stackrel{\leftrightarrow}{\boldsymbol{\sigma}}$ consists of normal and tangential components. Pressure, which is an isotropic force per unit area $p \hat{\mathbf{n}}$ exerted normal to any surface, is the most familiar normal stress. Viscous stresses $\stackrel{\leftrightarrow}{\sigma}_{v}$ $\sim \eta \nabla \mathbf{u}$ contain both normal and shear components. Additional stresses, both normal and shear, arise in complex fluids with deformable microstructure: polymer solutions, discussed in Sec. II.D, provide one example. Interfacial (capillary) stresses $\sigma_{c} \sim \gamma / R$, where $\gamma$ is surface tension and $R$ is surface curvature, are exerted normal to free fluid surfaces, whereas surface tension gradients give Marangoni stresses $\sigma_{m} \sim \nabla \gamma$ that are exerted along the surface. 
TABLE II. Physical properties of water at $20{ }^{\circ} \mathrm{C}$ and 1 atm pressure (Lide, 1999).

\begin{tabular}{lll}
\hline \hline Density & $\rho$ & $1.0 \mathrm{~g} / \mathrm{cm}^{3}$ \\
Shear viscosity & $\eta$ & $1.0 \times 10^{-2} \mathrm{~g} / \mathrm{cm} \mathrm{s}$ \\
Kinematic viscosity & $\nu$ & $1.0 \times 10^{-2} \mathrm{~cm}^{2} / \mathrm{s}$ \\
Surface tension (in air) & $\gamma$ & $73 \mathrm{~g} / \mathrm{s}^{2}$ \\
Thermal variation of $\gamma$ & $d \gamma / d T$ & $-0.15 \mathrm{~g} / \mathrm{s}^{2}{ }^{\circ} \mathrm{C}$ \\
Thermal diffusivity & $\kappa_{H}$ & $1.4 \times 10^{-3} \mathrm{~cm}^{2} / \mathrm{s}$ \\
Compressibility & $\alpha_{\rho}$ & $-4.6 \times 10^{-11} / \mathrm{ba}$ \\
Coefficient of thermal expansion & $\alpha_{T}$ & $2.1 \times 10^{-4} /{ }^{\circ} \mathrm{C}$ \\
\hline \hline
\end{tabular}

It is important to remember that fluids are made of molecules, and this discreteness reveals itself in the phenomenon of diffusion. Both the molecules of the fluid and any "tracers" or additives move diffusively through the fluid. A diffusivity arises for each tracer-the thermal diffusivity $\kappa_{H}$ for heat, the diffusivity $D$ $\sim k_{B} T / 6 \pi \eta a$ for spherical solute molecules of size $a$, and the kinematic viscosity $\nu=\eta / \rho$ for fluid vorticity. Representative values for these and other fluid properties are given in Table II.

\section{A. The Reynolds number: Inertial irrelevance}

Of all dimensionless numbers, the Reynolds number $(\mathrm{Re})$ is the one most often mentioned in connection with microfluidics. Ironically, it may also be the least interesting number for microfluidics: after all, almost without exception, microfluidic devices employ fluids with Reynolds numbers that are small enough for inertial effects to be irrelevant. Nevertheless, because low Reynolds number flows contradict day-to-day human experience with fluids, one must unlearn a lifetime's worth of high-Re intuition in order to effectively think about microfluidics. Purcell's description of this counterintuitive world, Life at low Reynolds number (Purcell, 1977) provides an excellent overview, and Brody et al. (1996) explore its biotechnological analog. More detailed accounts are given in conference proceedings (Batchelor, 1977; Hinch, 1988) and textbooks (Happel and Brenner, 1983; Kim and Karilla, 1991; Leal, 1992; Pozrikidis, 1992; Deen, 1998).

For the physicist more familiar with Newton's laws than with the convective derivative $\rho \mathbf{u} \cdot \nabla \mathbf{u}$ of the Navier-Stokes equations, Fig. 3 depicts two simple illustrations. The first involves a fluid that flows with velocity $U_{0}$ through a microchannel of width $w$ that makes a sudden right turn. During the turn time $\tau_{0} \sim w / U_{0}$, a fluid element rounding the corner loses momentum density $\rho U_{0}$ by exerting an inertial centrifugal force density $f_{i}$ $\sim \rho U_{0} / \tau_{0}=\rho U_{0}^{2} / w$. The other involves a fluid flowing in a channel that contracts over a length $l$. By mass conservation, the velocity increases as $u \sim U_{0}(1+z / l)$, causing a fluid element to gain momentum at a rate

$$
f_{i} \sim \rho \frac{d u}{d t}=\rho U_{0} \frac{d u}{d z} \sim \frac{\rho U_{0}^{2}}{l} .
$$

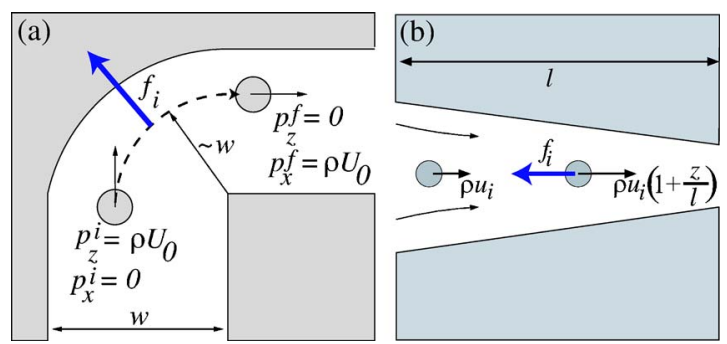

FIG. 3. (Color in online edition) Inertial forces exerted by accelerating fluid elements. (a) A small fluid element rounding a corner loses $\rho U_{0}$ of momentum during the time $\tau_{0} \sim w / U_{0}$ required to turn the corner, giving a centrifugal force density of order $f_{i} \sim \rho U_{0}^{2} / w$. (b) A fluid element flowing through a contraction with velocity gradient $d u / d z \sim U_{0} / l$ gains momentum density at a rate $f_{i} \sim \rho U_{0}^{2} / l$. In both cases, the inertial force exerted on the fluid is equal and opposite to the force required to accelerate each fluid element, and is the same for flows in either direction.

The force exerted on the rest of the fluid is equal and opposite to the force required to accelerate each fluid element. Thus the force on the fluid due to a curved streamline points outwards centrifugally, and the inertial force in an expansion or contraction points towards the wide end of the channel, regardless of the flow direction. An oscillatory flow through an asymmetric channel can drive a rectified, steady fluid motion which forms the basis for valveless diffuser pumps (Stemme and Stemme, 1993; Olsson et al., 1999).

In both cases, the magnitude of inertial and viscous force densities must be compared. Viscous force densities result from gradients in viscous stress, and thus scale as $f_{v} \sim \eta U_{0} / L_{0}^{2}$, where $L_{0}$ is a typical length scale. The ratio of these two force densities,

$$
\frac{f_{i}}{f_{v}}=\frac{\rho U_{0} L_{0}}{\eta} \equiv \mathrm{Re}
$$

is known as the Reynolds number.

Reynolds numbers for common microfluidic devices can be estimated as follows. With water as the typical working fluid, typical velocities of $1 \mu \mathrm{m} / \mathrm{s}-1 \mathrm{~cm} / \mathrm{s}$, and typical channel radii of $1-100 \mu \mathrm{m}$, the Reynolds numbers range between $\mathcal{O}\left(10^{-6}\right)$ and $\mathcal{O}(10)$. These low values of Re affirm that viscous forces typically overwhelm inertial forces, and the resulting flows are linear. The loss of nonlinearity at low Re led to the demise of the research effort devoted to miniaturized fluidic computation, whose fundamental elements relied upon inertial effects and thus could not scale down like their solidstate electronic competition (Humphrey and Tarumoto, 1965; Foster and Parker, 1970; Joyce, 1983).

When Re is very small, the nonlinear terms in Eq. (1) disappear, resulting in linear and predictable Stokes flow [Eq. (2)]. As Re increases, the first features of inertia become apparent. For example, in flow through a circular channel that is slightly curved with radius of curvature $R$ much larger than the channel radius $w$, centrifugal forces on fluid elements drive a secondary flow. The 


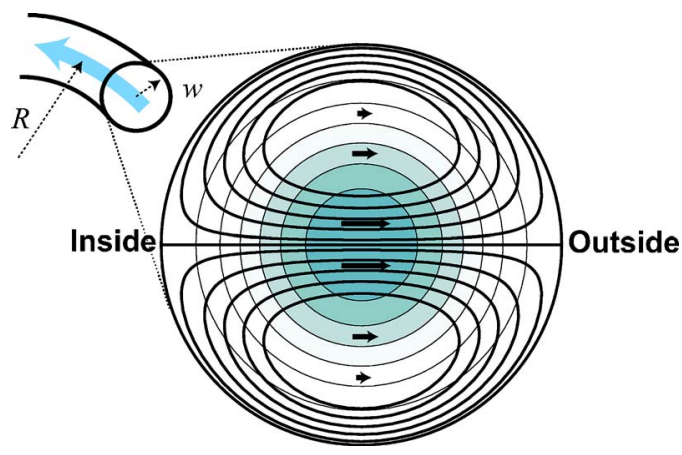

FIG. 4. (Color in online edition) A secondary Dean flow results from inertial forces on fluid flowing (into the page) through a slightly curved channel. Fluid elements are forced centrifugally towards the outside of the turn, and shaded contours show the magnitude of the centrifugal force density (greatest in the center, where the primary flow is fastest). This inhomogeneous forcing drives the secondary flow shown in the plane of the page.

no-slip boundary condition and inhomogeneous flow profile, $u \sim U_{0}\left[1-(r / w)^{2}\right]$, cause inhomogeneous centrifugal forcing, $f_{i} \sim U_{0}^{2}\left[1-(r / w)^{2}\right]^{2} / R$, and give the secondary "Dean flow" $U_{D} \sim \operatorname{Re}(w / R) U_{0}$ shown in Fig. 4 (Dean, 1927, 1928; McConalogue and Srivastava, 1968; Leal, 1992). Secondary flow in twisted pipes have served as a model system for mixing studies (Jones et al., 1989; Sharp et al., 1991; Jones and Young, 1994), and have recently been applied to create a passive, threedimensional "serpentine" micromixer (Liu et al., 2000; Rush et al., 2002, albeit with rectangular channels and appreciable Re). As Re gets still higher, the nonlinear inertial term destabilizes the flow, resulting in unpredictable, irregular turbulent flow. The standard example for the transition to turbulence involves flow through a straight circular pipe, where the transition occurs for $\mathrm{Re}$ between 2000 and 3000. Such Reynolds numbers are significantly higher than those encountered in microfluidic devices, and microfluidic flows generally fall safely within the laminar flow regime.

Thus far we have focused on steady inertial forcing due to the convective derivative $\rho \mathbf{u} \cdot \nabla \mathbf{u}$, which is almost always unimportant for microfluidic flows because its time dependence arises from $U_{0}$. The linear unsteady term $\rho \partial \mathbf{u} / \partial t$, on the other hand, sets the inertial time scale $\tau_{i}$ required to establish steady flows. This time scale can be estimated by balancing the unsteady inertial force density $\sim \rho U_{0} / \tau_{i}$ with the viscous force density $\eta U_{0} / L_{0}^{2}$, giving

$$
\tau_{i} \sim \frac{\rho L_{0}^{2}}{\eta}
$$

The inertial time scale can be interpreted as the time required for vorticity to diffuse a distance $L_{0}$, with diffusivity $\nu=\eta / \rho$. This time scale is typically small, and pressure-driven flow requires $\tau_{i} \sim 10 \mathrm{~ms}$ to reach steady state in rigid $100-\mu \mathrm{m}$ channels. However, inertial effects from rapidly oscillating flows can give rise to the steady streaming flow discussed in Sec. III.B.

Inertia rarely plays a significant role in microfluidic systems, and as systems are made ever smaller, it becomes even less relevant. Without the inertial nonlinearity, straightforward microfluidic systems have regular, deterministic flow. There do exist, however, many physical processes exploited in microfluidics-capillary effects at free surfaces, viscoelasticity in polymer solutions, and electrokinetic effects, to name a few-whose nonlinearities may even increase as device dimensions diminish, and which give rise to a rich variety of microfluidic phenomena. The remainder of this review explores this world.

\section{B. The Péclet number and the problem of mixing}

In the everyday high-Re world, random eddies continuously churn the fluids in which we live-and in the process, stretch and fold fluid elements chaotically. As a result of this so-called turbulent mixing, gradients are significantly enhanced and time scales for mixing are dramatically reduced. In fact, without turbulent mixing or thermal convection, it would take about a year to smell your feet after taking off your shoes! The laminar fluid flows that naturally arise in the low-Re world of microfluidics, however, force mixing to occur by diffusion alone, which can result in unacceptably long mixing times of order minutes or more.

Purely diffusive mixing can be desirable or not, depending on the application. Microfluidic chemical reactors require different solutions to be brought together and mixed rapidly, allowing the dynamics of the reactions to be probed, rather than the diffusive dynamics of the molecules themselves. The opposite problem is faced, however, in sorting and analyzing the products of those same reactions: the faster the mixing, the harder the separation. Controlling dispersion in microfluidic devices, then, is often of paramount importance.

For example, consider a $\mathrm{T}$ junction in which two fluids are injected to flow alongside each other, as in Fig. 5(a). How far down the channel must the fluids flow before the channel is homogenized? A simple estimate requires the particles or molecules to diffuse across the entire channel, giving a time $\tau_{D} \sim w^{2} / D$, where $w$ is the width of the channel. During this time, the stripe will have moved a distance $Z \sim U_{0} w^{2} / D$ down the channel, so that the number of channel widths required for complete mixing would be of order

$$
\frac{Z}{w} \sim \frac{U_{0} w}{D} \equiv \mathrm{Pe} .
$$

The dimensionless number on the right is known as the Péclet number $(\mathrm{Pe})$, which expresses the relative importance of convection to diffusion. In this example, the number of channel widths required for full mixing varies linearly with Pe. Using the diffusivities in Table III, we see that even a small protein flowing with the fluid through a $100-\mu \mathrm{m}$ channel at $100 \mu \mathrm{m} / \mathrm{s}$ requires $\mathrm{Pe}$ 
(a)
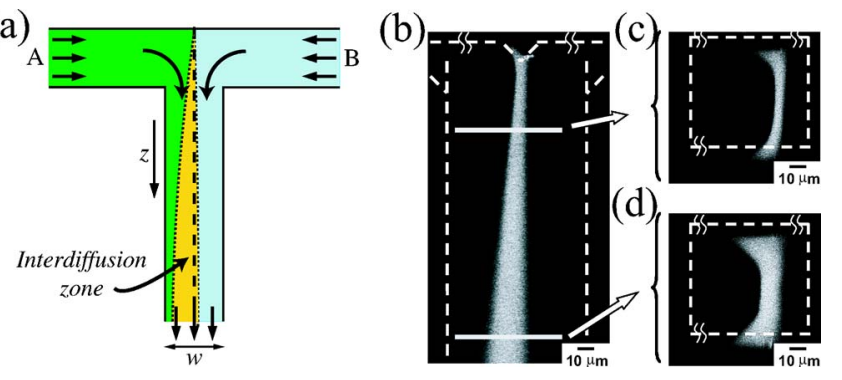

FIG. 5. (Color in online edition) (a) The microfluidic T sensor (Kamholz et al., 1999). Different fluids are brought together at a $\mathrm{T}$ junction to flow alongside each other down the channel. A simple estimate suggests that the interdiffusion zone spreads diffusively, with the square root of time (or downstream distance), although (b)-(d) show this naive argument to break down near the "floor" and "ceiling" of the channel. Confocal microscopy reveals the three-dimensional nature of the spreading of the interface in the $\mathrm{T}$ sensor (Ismagilov et al., 2000). (b) Fluorescent tracers mark reactions occurring in the interdiffusion zone, here seen from above. (c), (d) The no-slip nature of the top and bottom walls of the channel affect the flow profile, so that tracer molecules near the boundaries diffuse and spread with $z^{1 / 3}$, rather than $z^{1 / 2}$. Reprinted with permission from Ismagilov et al., 2000. (C2000, AIP.

$\sim 250$ channel widths (approximately $2.5 \mathrm{~cm}$ and $4 \mathrm{~min}$ ) to completely mix.

\section{Sensing, filtering, and fabricating with parallel laminar flows}

The competition between convection and diffusion, embodied in the Péclet number, forms the basis for a number of techniques for sensing and separating flow ingredients. The following devices are designed to operate at intermediate Pe, where differences in solute diffusion rates play the key role.

\section{a. $T$ sensor}

One device that employs adjacent laminar streams is the T sensor, shown in Fig. 5(a) (Kamholz et al., 1999; Weigl and Yager, 1999). Two fluid streams are brought to flow alongside each other down a channel, and solute molecules in each stream diffuse into the other, forming an interdiffusion zone whose boundary is measured, typically with a fluorescent marker. (Note, however, that

TABLE III. Typical diffusivities for various tracers in water at room temperature.

\begin{tabular}{|c|c|c|}
\hline \multicolumn{3}{|c|}{ Characteristic diffusivities } \\
\hline Particle & Typical size & Diffusion constant \\
\hline Solute ion & $10^{-1} \mathrm{~nm}$ & $2 \times 10^{3} \mu \mathrm{m}^{2} / \mathrm{s}$ \\
\hline Small protein & $5 \mathrm{~nm}$ & $40 \mu \mathrm{m}^{2} / \mathrm{s}$ \\
\hline Virus & $100 \mathrm{~nm}$ & $2 \mu \mathrm{m}^{2} / \mathrm{s}$ \\
\hline Bacterium & $1 \mu \mathrm{m}$ & $0.2 \mu \mathrm{m}^{2} / \mathrm{s}$ \\
\hline Mammalian/human cell & $10 \mu \mathrm{m}$ & $0.02 \mu \mathrm{m}^{2} / \mathrm{s}$ \\
\hline
\end{tabular}

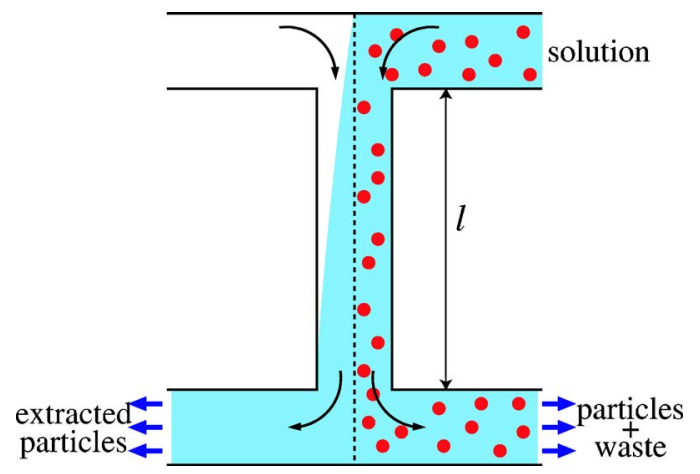

FIG. 6. (Color in online edition) The membraneless $\mathrm{H}$ filter exploits the different rates at which tracers with different diffusivities (and thus Pe) spread across a channel. The length $l$ is chosen so that large waste products do not have time to diffuse across the channel, and thus remain confined to their initial stream, whereas smaller molecules of interest diffuse across the channel into the neighboring stream. At the outlet, the second stream contains the more mobile species almost exclusively (Brody et al., 1996; Brody and Yager, 1997).

reactions introduce additional time scales and complexity into the system.) $\mathrm{T}$ sensors have be used to measure analyte concentration (Weigl and Yager, 1999) and analyte diffusivities and reaction kinetics (Kamholz et al., 1999, 2001; Baroud et al., 2003). Finally, competitive immunoassays have been performed by injecting an antibody solution alongside a solution of known, labeled antigen. Antigen-antibody binding is evidenced by marker accumulation in the interdiffusion zone, and an unmarked antigen can be detected differentially, as competition for antibody binding alters the marker profile measured downstream (Hatch et al., 2001).

The naive picture presented above does not tell the whole story about the $\mathrm{T}$ sensor, however. Ignoring the channel top and bottom leads to the expectation that the width of the interdiffusion zone should grow with $z^{1 / 2}$, as in Eq. (7). In fact, experiment and analysis have shown the front to behave in a more complicated fashion. Confocal microscopy of the three-dimensional front (Fig. 5) determined the spreading near the top and bottom walls to vary with $z^{1 / 3}$ and with $z^{1 / 2}$ near the middle (Ismagilov et al., 2000), which occurs because fluid near the top and bottom moves more slowly than the middle, so that tracers do not move as far downstream as they diffuse across the streamlines. The near-wall interfacial dynamics can thus be understood in terms of the classic Lévêque problem of diffusion in a shear flow (Lévêque, 1928; Deen, 1998; Ismagilov et al., 2000; Kamholz and Yager, 2001). Further analysis near the inlet reveals $z^{1 / 3}$ spreading near the walls and $z^{1 / 2}$ in the channel center, which then catches up downstream (Kamholz and Yager, 2002).

\section{b. Filtration without membranes}

Figure 6 depicts the $\mathrm{H}$ filter, a simple device that filters particles by size without a membrane (Brody et al., 1996; Brody and Yager, 1997). As in the T sensor, two different streams are brought together to flow alongside 
(a)

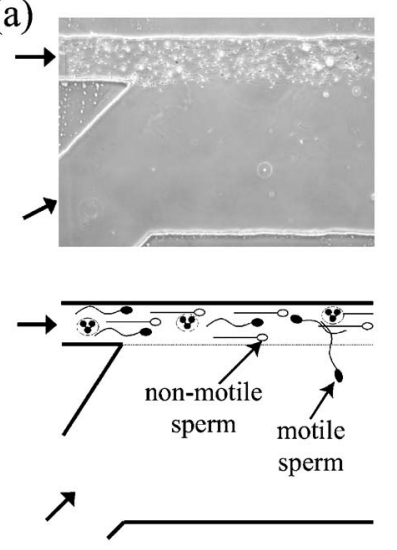

(b)

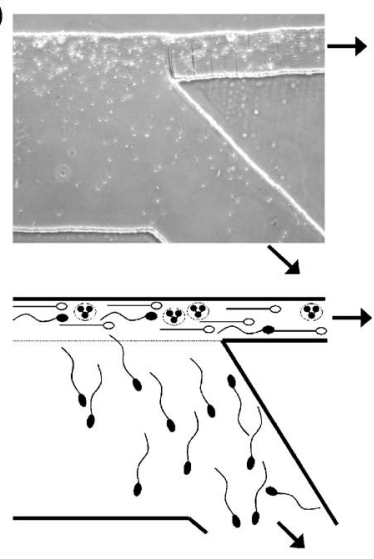

FIG. 7. A variant of the $\mathrm{H}$ filter (Fig. 6) that separates motile from nonmotile sperm. While the traditional $\mathrm{H}$ filter relies upon particle diffusivity differences for differentiation, this device exploits the fact that motile sperm disperse across and homogenize the channel much more rapidly than nonmotile ones, which spread via diffusion alone. Adapted and reprinted with permission from Cho, Schuster, et al., 2003. (C)2003, American Chemical Society.

each other down a channel. One stream is a dilute solution of different-sized molecules or particles, each of which has its own diffusivity and Péclet number. Using the simple argument above, each Pe determines the channel length required for that component to diffuse across the channel width. The $\mathrm{H}$ filter works when the length $l$ or flow velocity $U_{0}$ is chosen so that one Pe is small and the other is large. Low-Pe small solute particles diffuse to fill the channel before exiting, whereas high-Pe large particles remain confined to their half of the channel. A reverse $\mathrm{T}$ sensor then reseparates the flow into two streams, of which one contains mostly small particles, and the other contains both species.

The $\mathrm{H}$ filter requires only that the components to be separated spread at different rates transverse to the flow, and works regardless of the cause of the difference in dispersivity. Figure 7 shows a variant on the $\mathrm{H}$ filter, wherein motile sperm are separated from nonmotile ones (Cho, Schuster, et al., 2003). Rather than differing in diffusivity, motile sperm rapidly and randomly swim to fill the channel, as compared with nonmotile sperm, which spread via diffusion alone. Here as above, the species that spreads quickly is extracted.

A device by Schilling et al. (2002) demonstrates the type of analysis that can be performed with these principles. An $\mathrm{H}$ filter brings a stream of cells into contact with a chemical agent that lyses the cell membrane, allowing certain intracellular molecules to escape. One output of the $\mathrm{H}$ filter contains both waste cells and intracellular molecules. The other contains mostly the molecules of interest, which are then brought to flow alongside a stream of detection molecules in a $\mathrm{T}$ sensor to probe various properties, e.g., identification or concentration.

(a)

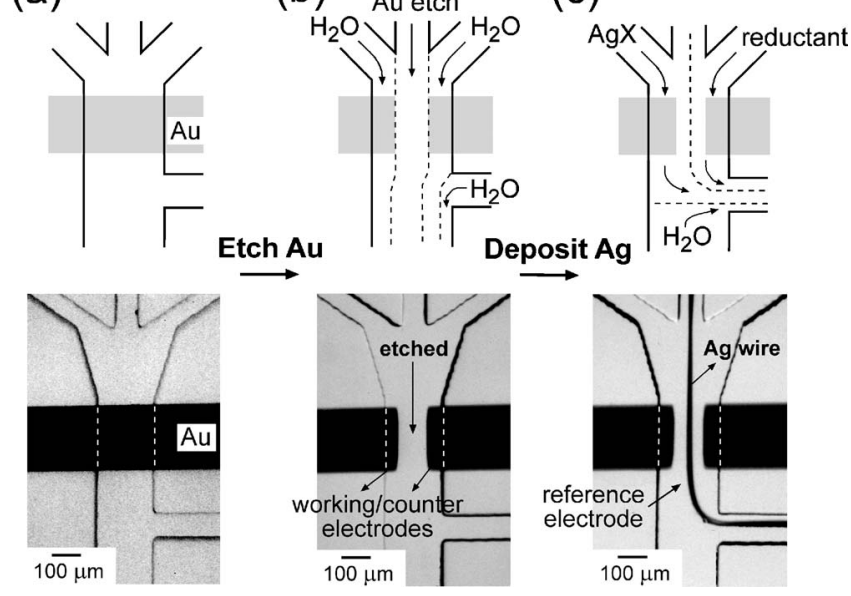

FIG. 8. A three-electrode system fabricated within existing microchannels. (a) Initially, a gold strip runs perpendicular to a microchannel. (b) Three high-Pe fluid streams flow down the channel, of which the central stream contains a chemical that etches away the underlying gold and separates the two electrodes. (c) Finally, a reaction at the interface of two high-Pe fluid streams causes silver to precipitate and form a thin wire on the floor of the channel. Reprinted with permission from Kenis et al., 1999. (C1999, AAAS.

\section{c. Fabricating and interrogating using multiple laminar streams}

The above devices work in the intermediate-Pe regime: the $\mathrm{T}$ sensor requires the interface to spread diffusively on appropriate time and length scales, and the $\mathrm{H}$ filter is optimal when $\mathrm{Pe}$ is small for the component to be extracted, and large for the "waste." We now discuss devices and techniques that are optimal in the large-Pe regime, wherein multiple fluid streams can flow alongside each other over long distances with minimal mixing.

Nonmixing, high-Pe flows can be used to fabricate and etch structures within existing microchannels (Takayama et al., 1999, 2001; Kenis et al., 2000). For example, Fig. 8 shows the fabrication of a three-electrode system within a microchannel (Kenis et al., 1999), in which parallel streams selectively etch areas of an electrode, after which a silver wire is formed by a precipitation reaction between two streams. Other fabrication techniques include selectively patterning hydrophobic stripes along a channel (Zhao et al., 2001), cutting trenches into channel walls using etchants, selectively coating portions of channel walls with molecules or cells, and forming linear deposits of crystals, polymeric lines, and thin wires within an existing channel (Kenis et al., 1999).

By growing or placing living cells within microchannels, cellular behavior and response can be probed locally if different laminar streams affect different parts of the cell. For example, the stream to the left of the dashed line in Fig. 9 contains trypsin, which disrupts cellsubstrate adhesions and causes a bovine capillary endothelial cell to lose its local footing and move to the right (Takayama et al., 2003). Similarly, locally stimulating receptors allows the study of cell signaling (Sawano et al., 

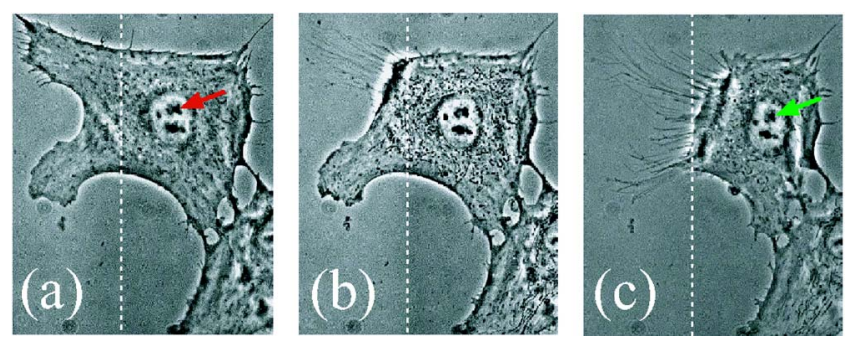

FIG. 9. (Color in online edition) Local cell manipulation with parallel laminar streams. The stream to the left of the dashed line contains trypsin, which disrupts cell adhesion and causes a bovine capillary endothelial cell to recoil towards the "inert" stream on the right. (a) Trypsin starts, (b) $1 \mathrm{~min}$; (c) $3.5 \mathrm{~min}$. Reprinted with permission from Takayama et al., 2003. (C2003, Elsevier.

2002), selectively disrupting actin filaments probes local changes in cytoskeletal structure, and selectively labeling mitochondria at opposite ends of a cell allows their motion to be traced (Takayama et al., 2001, 2003).

\section{Fighting diffusion: Particle separations}

Molecular separation techniques in analytical chemistry were among the earliest applications of microfluidic devices (Harrison et al., 1992, 1993; Manz et al., 1993; Reyes et al., 2002). Many techniques exist to separate the components of solution for analysis, typically by size or electric charge [see, e.g., Giddings (1991)]. For example, measurements in molecular biology and genomics often require DNA fragments to be sorted by size, which is typically done electrophoretically. Differentsized fragments travel with relative velocities rates $\Delta U$ and spread diffusively with diffusivity $D$. (Note that $D$ reflects molecular diffusivity as well as Taylor dispersivity, as discussed below.) A mixed collection of fragments evolves into "bands" separated by a distance $\Delta Z \sim \Delta U t$, and whose widths grow as $\Delta W \sim(D t)^{1 / 2}$. Assuming the different bands to travel with velocity scale $U_{0}$, an estimate for effective separation can be obtained. To clearly resolve the bands, the separation between bands must greatly exceed the width of each, which requires

$$
\Delta U t \gg \sqrt{D t} \rightarrow\left(\frac{U_{0} Z}{D}\right)^{1 / 2} \gg 1
$$

Here we have assumed $\Delta U \approx \mathcal{O}\left(U_{0}\right)$ and converted time into downstream distance via $Z=U_{0} t$. Another Péclet number, $\mathrm{Pe}=U_{0} Z / D$, thus appears, which is called the theoretical number of plates in the separation literature (Giddings, 1991). Since $U_{0} \propto E$ in electrophoretic separations, Eq. (8) indicates that strong electric fields and long channels are best for separation.

We close our discussion of intermediate-Pe effects with a final example for the separation of suspended particles by size (Duke and Austin, 1998; Ertaş, 1998; Chou et al., 1999; Austin et al., 2002; Cabodi et al., 2002; Huang, Silberzan, et al., 2002), related to theoretical ideas involving "ratchet" potentials (Ajdari and Prost,
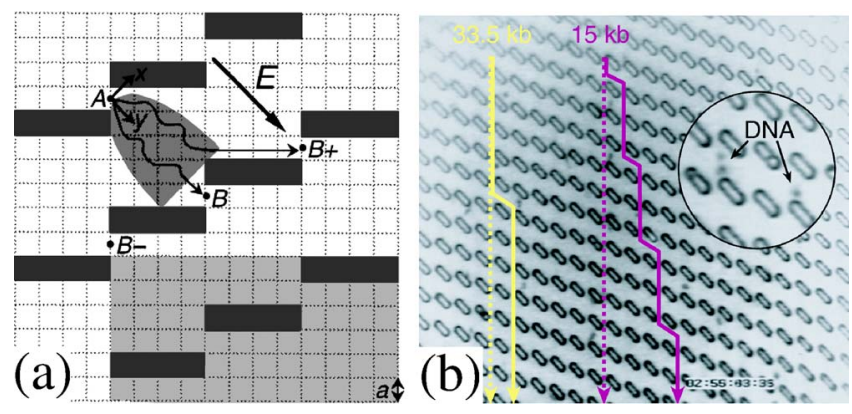

FIG. 10. (Color in online edition) Sorting by diffusion. To leading order, particles driven through the array follow streamlines, and travel across streamlines through diffusion alone. (a) When the array has a broken spatial symmetry, the hopping rates are biased in one direction. Reprinted with permission from Duke and Austin, 1998. (b) Smaller DNA fragments hop more frequently and thus follow trajectories that are more transversely directed than larger particles. Reprinted with permission from Chou et al., 2000. @2000, Wiley.

1992; Magnasco, 1993; Astumian and Bier, 1994; Prost et al., 1994; Bier and Astumian, 1996). Figure 10 shows microfabricated arrays of asymmetric obstacles, through which particles are driven electrophoretically or with pressure-driven flow. In the absence of diffusion (i.e., Pe $\gg 1$ ), suspended particles follow field or flow lines. At moderate Pe, however, a particle occasionally diffuses far enough to cross over into a neighboring trajectory in a direction biased by the array's broken symmetry (Duke and Austin, 1998; Ertaş, 1998). The hopping rate of each species increases as its Pe decreases, so that smaller (higher- $D$, lower-Pe) particles make lateral hops more frequently, and thus separate by size. However, Austin et al. (2002) and Huang, Silberzan, et al. (2002) argued that this simple picture was insufficient for separation, and one of two criteria must be met: (i) field and/or flow lines must penetrate the obstacles, or (ii) particles must be comparable in size to the gaps between obstacles. The latter allows for separations even in the absence of diffusion ( $\mathrm{Pe} \rightarrow \infty$; Huang et al., 2004). The former has been achieved in electrophoretic separations by using slightly conducting posts (Huang, Silberzan, et al., 2002), and in "optical fractionization" wherein focused laser arrays impede particles but not fluid flow (MacDonald et al., 2003; Gluckstad, 2004; Ladavac et al., 2004).

\section{Beating diffusion: When mixing matters}

Thus far, we have concentrated on parallel laminar streams, where interfaces between fluids of different content are largely parallel to the fluid velocity. This strategy was chosen because the mixing of the two streams occurs slowly enough to be monitored, manipulated, measured, or even ignored. As such, fluid motion plays a minimal role in the evolution of the interface. The first hint that we have seen of the role convection plays in interface evolution appeared in Fig. 5, where the parabolic velocity profile modified the scaling of the interface spreading from $\mathrm{Pe}^{1 / 2}$ to $\mathrm{Pe}^{1 / 3}$ near the $\mathrm{T}$ junction. 
In some applications involving inhomogeneous fluids, however, the side-by-side configuration of parallel laminar streams is not possible. This occurs when molecules or particles are separated by size, since separation bands are typically oriented perpendicular to the flow or field direction. In these cases, convection invariably acts to fight the separation process.

Many applications, moreover, require exactly the opposite: a method to rapidly mix an inhomogeneous solution. To probe reaction kinetics or protein folding, for example, distinct fluids must be mixed rapidly enough that the system is reaction limited, rather than diffusion limited. Since mixing times or distances can be very long, strategies are required to enhance mixing in microdevices. All mixing-whether in turbulent or laminar flows-ultimately occurs due to molecular diffusion. Fluid stirring can be used to stretch and fold inhomogeneous fluid blobs until mixing (diffusive migration across streamlines) occurs [following the terminology of Eckart (1948) and Aref and Balachandar (1986)]. The basic idea behind mixers is to use stirring motions to reduce the distance over which mixing must occur.

A simple and elegant method for enhancing mixing rates by reducing mixing distances involves flow focusing (as in Fig. 14, but without capillary effects), in which a central stream is narrowed to a width $w$ using an outer flow. In so doing, diffusive penetration of molecules from the outer flow into the central stream occurs more rapidly (Knight et al., 1998; Pabit and Hagen, 2002). Such an approach has been used to study protein folding on submillisecond time scales (Pollack et al., 1999, 2001; Kauffmann et al., 2001; Pabit and Hagen, 2002; Lipman et al., 2003); see Eaton et al. (2000) for an early review. A related approach reduces the diffusion length by forcing the solution through a packed bed of microbeads (Seong and Crooks, 2002).

More elaborate methods are required, however, to homogenize the contents of a microfluidic channel or chamber. The classic text of Ottino (1989) provides an excellent introduction to mixing science. Various strategies are being developed to enhance microfluidic mixing, and a recent issue of Philosophical Transactions has been dedicated entirely to microfluidic mixing. For this issue, Ottino and Wiggins (2004) provide an overview, Wiggins and Ottino (2004) explore the mathematical foundations of mixing, Stremler et al. (2004) discuss design principles, and Bringer et al. (2004), Bottausci et al. (2004), Campbell and Grzybowski (2004), Darhuber et al. (2004), Mensing et al. (2004), Stroock and McGraw (2004), and Tabeling et al. (2004) explore different mixing systems in depth.

It is of great importance to understand the role of fluid convection in the dispersion of tracers, by which we mean any object that is transported by, and diffuses in, the fluid (examples include dyes, analyte molecules, proteins, cells, salt, or even heat). A pattern will emerge: tracer dispersion is initially dominated by convective stretching with the fluid, which is then followed some time later by diffusive homogenization.

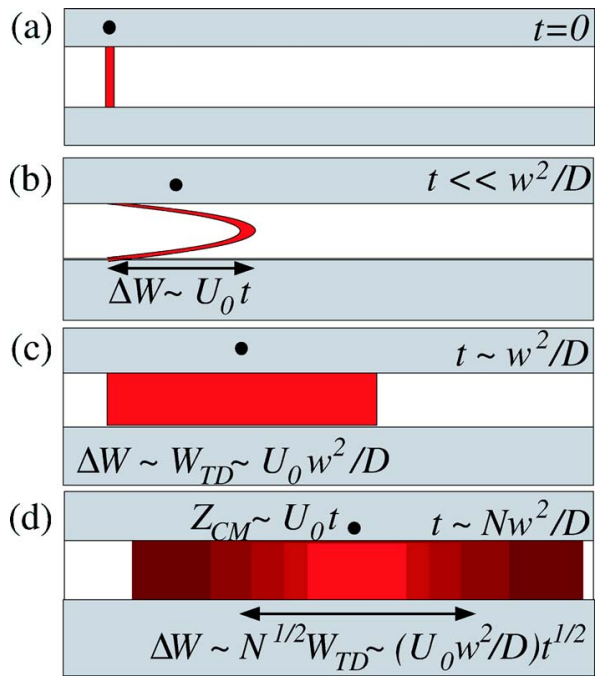

FIG. 11. (Color in online edition) Taylor dispersion. (a) An infinitesimally thin tracer stripe in a circular channel of radius $w$. (b) Under a pressure-driven flow, the stripe is convectively stretched into a parabolic shape whose width $\Delta W$ increases linearly with time. (c) After a characteristic time scale $\tau_{D}$ $\sim w^{2} / D$ for tracers to diffuse across the channel, the parabola is "smoothed" into a plug of width $W_{T D} \sim U_{0} w^{2} / D$. (d) The plug can be broken into many thin stripes, each of which subsequently undergoes processes (a)-(c) to be smoothed into plugs of width $W_{T D}$ on a time scale $\tau_{D}$. A tracer thus takes a random step of size $\lesssim W_{T D}$ on each time step $\tau_{D}$, causing the stripe to evolve as a Gaussian spreading with effective diffusivity $D_{z} \sim U_{0}^{2} w^{2} / D$.

\section{a. Taylor dispersion}

Taylor dispersion provides a classic example of the role of convection in dispersing inhomogeneous flows (Taylor, 1953, 1954). A thin stripe of tracers initially spans a circular channel of radius $w$, as in Fig. 11(a). In the absence of diffusion, pressure-driven (Poiseuille) flow with profile $u_{z}=U_{0}\left(1-r^{2} / w^{2}\right)$ stretches the stripe into a parabola, where the tracer in the center leads the tracer at the walls by a distance $U_{0} t$. In a reference frame that moves with the mean flow, the tracer stripe is stretched to $\pm \sim U_{0} t$ [Fig. 11(b)]. However, there is a time scale $\tau_{D} \sim w^{2} / D$ at which molecular diffusion across the channel smears the parabolic stripe into a plug of width $W_{T D} \sim U_{0} w^{2} / D$ [Fig. 11(c)]. Thus a tracer within the initial stripe effectively takes a random step of size $\lesssim W_{T D}$ each time step $\tau_{D}$. Each stripe within the plug goes through the same process: each is convectively stretched and then diffusively smeared to a width $W_{T D}$ after a time $\tau_{D}$. After $N$ steps, the initially thin stripe evolves into a Gaussian with width

$$
\left\langle W^{2}\right\rangle^{1 / 2} \sim N^{1 / 2} W_{T D} \sim\left(\frac{U_{0}^{2} w^{2}}{D} t\right)^{1 / 2}
$$

The tracer distribution thus grows diffusively (as $t^{1 / 2}$ ) with an effective long-time axial diffusivity, 


$$
D_{z} \sim \frac{U_{0}^{2} w^{2}}{D} \sim \operatorname{Pe}^{2} D
$$

that occurs in addition to the molecular diffusivity $D$. Detailed analyses for specific geometries yields prefactors, as presented in textbooks such as Brenner and Edwards (1993) and Deen (1998). Important examples include flow in a circular channel with radius $w$, for which $D_{z}=\bar{U}^{2} w^{2} / 48 D$; flow in shallow rectangular channel of height $h$, for which $D_{z}=\bar{U}^{2} h^{2} / 210 D$; and simple shear flow between plates separated by $h$, for which $D_{z}$ $=2 \bar{U}^{2} h^{2} / 15 D$.

Several important points are in order. First, the axial Taylor dispersivity $D_{z}$ [Eq. (10)] is only valid on long time scales $t \gg w^{2} / D$, or after a downstream distance $L$ $\gg$ Pe $w$. This is sometimes impractically long for microfluidic applications-Taylor's original experiments were conducted using a $500-\mu \mathrm{m}$ capillary and lasted four hours! On shorter time scales, tracer blobs are stretched convectively, and grow linearly in time. Only after tracers have had enough time to diffuse across the channel does a blob spread diffusively with dispersivity $D_{z}$ [Eq. (10)]. Taylor dispersion acts in the direction of the flow, and is thus not observed in $\mathrm{T}$ sensors and $\mathrm{H}$ filters, where gradients in tracer concentration are perpendicular to the flow direction. Second, Taylor dispersion may appear to dramatically enhance dispersion for small solutes, since $D_{z} \sim D^{-1}$. Without Taylor dispersion, however, convection would stretch tracer blobs indefinitely with time; Taylor dispersion in fact cuts off the convective growth and reduces it to diffusive $\left(t^{1 / 2}\right)$ growth. Thus stripes in pressure-driven flow evolve into Gaussian bands moving at the mean flow speed and spreading with an effective diffusivity $D+D_{z}$. In fact, in sufficiently slow flows and/or thin channels for which Pe $\leqslant 1$, tracers in pressure-driven flow will evolve as though the flow were uniform (e.g., like electro-osmotic "plug" flow, Sec. III.A).

A vast literature has developed around Taylor dispersion, with over 2000 citations to date. Following Taylor's original exploration of the subject (Taylor, 1953, 1954), Taylor's picture was generalized using a method of moments that allows for arbitrary channel shapes and flows (Aris, 1956, 1959), and generalized Taylor dispersion theory was developed (Brenner and Edwards, 1993).

\section{b. Rotary mixer}

Above we developed the basic picture of Taylor dispersion, wherein initially convective stretching of tracers eventually gave way to diffusive dispersion, with enhanced axial dispersivity. We now examine how convective stretching can be exploited to enhance mixing of inhomogeneous fluids in microfluidic devices. The general theme to keep in mind is that there are typically two steps: (i) tracer blobs are initially stretched convectively by the flow, and (ii) molecular diffusion of the tracers across fluid streamlines homogenizes the flow once gra-

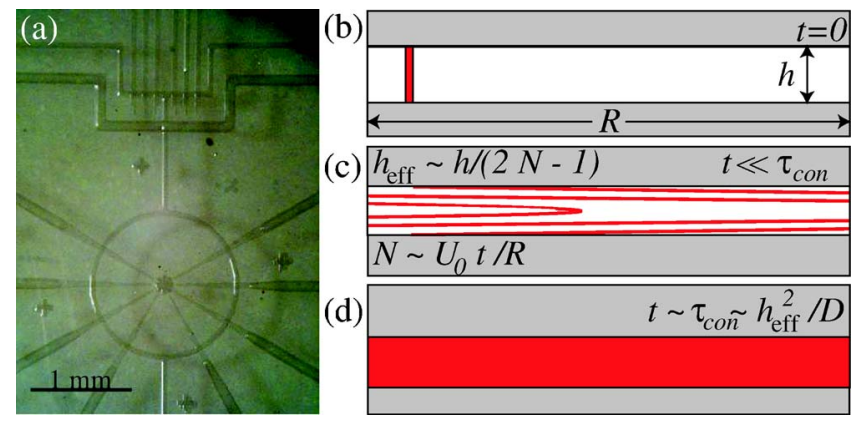

FIG. 12. (Color in online edition) Rotary mixer (a), in which valves [Figs. 45(a) and 45(b)] pump peristaltically around the ring. Reprinted from Chou et al., 2001, with kind permission of Springer Science and Business Media, (C2001. In the convectively stirred regime, an initially vertical stripe (b) deforms under the parabolic flow (c) to make $N=U_{0} \tau_{s} / 2 \pi R$ revolutions, reducing the effective distance between branches of the stripe to $h_{\text {eff }} \sim h / 2 N$. The stripe continues to roll up, and $h_{\text {eff gets }}$ increasingly smaller, until (d) molecular diffusion across $h_{\text {eff }}$ is fast enough to homogenize the channel. This requires a time $\tau_{\text {con }} \sim \mathrm{Pe}^{-2 / 3} \tau_{D}$, where $\tau_{D} \sim h^{2} / D$.

dients are sufficiently steep or time scales sufficiently long.

As a paradigmatic example, we investigate the rotary mixer shown in Fig. 12(a) (Chou et al., 2001). For simplicity, we use physical arguments and treat a twodimensional model, similar to mixing in a slit channel (Handique and Burns, 2001). A more mathematical treatment is provided by Gleeson et al. (2004). Fluid is pumped with average velocity $U_{0}$ around a closed, circular channel of radius $R$ and height $h$. How quickly the fluid mixes depends on how quickly the fluid flows. There are three distinct regimes: (i) diffusion dominated, (ii) Taylor dispersion mediated, and (iii) convectively stirred.

(i) Diffusion-dominated mixing. When the pumping velocity $U_{0}$ is extremely slow $\left(\mathrm{Pe}=U_{0} h / D \ll 1\right)$, molecular diffusion plays the dominant role in tracer dispersion, beating even Taylor dispersion $\left(D \gg D_{z}\right)$. In this case, mixing is achieved only after tracers diffuse around the circumference of the ring, requiring a time $\tau_{R}$,

$$
\tau_{R} \sim \frac{(2 \pi R)^{2}}{D}=\left(\frac{2 \pi R}{h}\right)^{2} \tau_{D}
$$

Note the mixing time does not depend on $U_{0}$ or therefore on Pe.

(ii) Taylor dispersion-mediated mixing. If the flow rate is increased so that $\mathrm{Pe} \gg 1$, Taylor dispersion assumes the dominant role in axial dispersion. In this case, axial spreading increases diffusively with Taylor dispersivity $D_{z}$ [Eq. (10)], resulting in a mixing time

$$
\tau_{T D} \sim \frac{(2 \pi R)^{2}}{D_{z}} \sim \frac{D(2 \pi R)^{2}}{U_{0}^{2} h^{2}} \sim \mathrm{Pe}^{-2} \tau_{R} .
$$

In order for Taylor dispersion to dominate, molecules must diffuse across the channel (requiring $\tau_{D} \sim h^{2} / D$ ) before convective stretching folds a stripe into itself (re- 
quiring $\tau \sim 2 \pi R / U_{0}$ ). Taylor-dispersion-mediated mixing thus occurs for $\mathrm{Pe}$ in the range $1 \ll \mathrm{Pe} \ll 2 \pi R / h$.

(iii) Convectively-stirred mixing. Finally, when the flow rate is sufficiently rapid $(\mathrm{Pe} \gg 2 \pi R / h)$, a tracer stripe is folded into itself multiple times before molecules diffuse across the channel. After a stirring time $\tau_{s}$, fluid in the center makes $N=U_{0} \tau_{s} / 2 \pi R$ full revolutions. A vertical stripe is stretched by the flow into a parabola, which folds over itself $N$ times, and the distance between adjacent stripes decreases as $h_{\text {eff }} \sim h / 2 N$ [Fig. 12(b)]. For full mixing to occur, molecules in the stripe need only diffuse across the reduced distance $h_{\text {eff }}$, which requires a time $\tau_{h} \sim h_{\text {eff }}^{2} / D$. Stirring proceeds until mixing wins, which occurs when the two time scales roughly coincide: $\tau_{s}$ $\sim \tau_{h}=\tau_{\text {con }}$,

$$
\begin{aligned}
& N_{\text {con }} \sim\left(\frac{h}{\pi R}\right)^{1 / 3} \mathrm{Pe}^{1 / 3}, \\
& \tau_{\text {con }} \sim \mathrm{Pe}^{-2 / 3}\left(\frac{\pi R}{h}\right)^{2 / 3} \tau_{D} \sim \mathrm{Pe}^{-2 / 3}\left(\frac{h}{\pi R}\right)^{4 / 3} \tau_{R} .
\end{aligned}
$$

Before continuing, we note a few points about the rotary mixer. First, mixing or dispersion is only enhanced across flow gradients, just as with Taylor dispersion. Therefore, the rotary mixer is most effective in batch processes, in which a homogeneous plug is mixed thoroughly into a fluid stored in the ring. Examples where batch process are mixed in this manner include protein solubility studies (Hansen et al., 2004) and single-cell DNA extraction and amplification (Hong et $a l ., 2004)$. Second, in the convectively stirred mixing regime $(\mathrm{Pe} \gg R / h)$, the number of cycles required for mixing increases algebraically with $\mathrm{Pe}$, because fluid elements are stretched linearly in time. Below we will see the number of cycles required for chaotic mixers, which stretch fluid elements exponentially in time, increases logarithmically with Pe. In all cases, the time per cycle varies like $\mathrm{Pe}^{-1}$, giving a mixing time that decreases algebraically with Pe.

\section{c. Mixing by chaotic advection}

When fluid elements move chaotically, they are stretched and folded exponentially, enhancing mixing. Turbulent mixing works on this principle. Although Stokes flows are inherently linear, even simple Stokes flows can have chaotic streamlines that exponentially stretch and fold. This forms the basis for Aref's classic mixing strategy, chaotic advection (Aref, 1984, 2002).

The staggered herringbone mixer is a chaotic mixer for continuous flow systems that does not depend on inertia (Stroock, Dertinger, Ajdari, et al., 2002; Stroock and McGraw, 2004). In it asymmetric grooves in the channel walls induce an axially modulated secondary flow (Stroock, Dertinger, Whitesides, et al., 2002) in the form of an asymmetric set of counter-rotating fluid rolls (Fig. 13). The asymmetry of the rolls is periodically reversed, so that the distance between stripes halves with each cycle, leading to exponential stretching and folding of

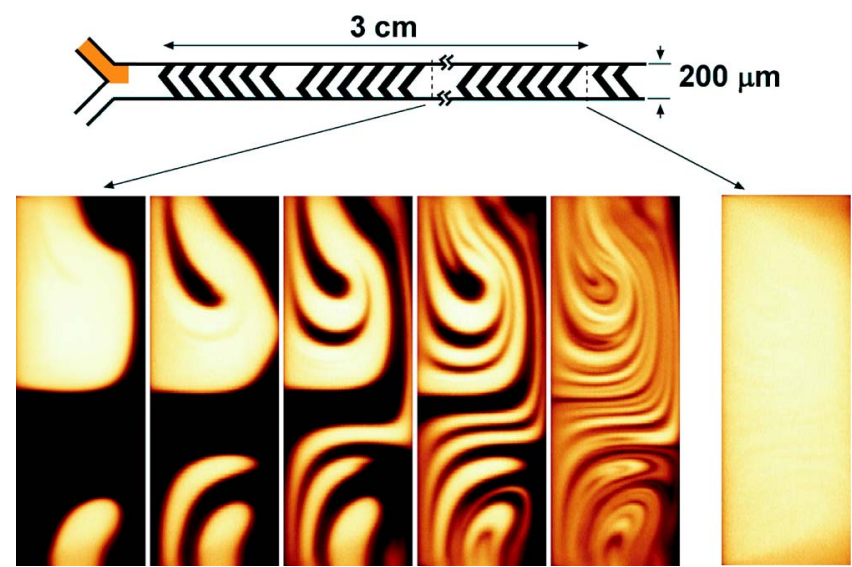

FIG. 13. (Color in online edition) Continuous-flow staggered herringbone mixer, in which grooved channel walls drive alternating, asymmetric helical secondary flows that chaotically stir the fluid. Each cycle cuts the distance between stripes in half, so that the distance between stripes decreases exponentially with the number of cycles. Diffusive mixing occurs when the tracer can diffuse from one stripe to the next before another cycle has occurred, giving a mixing time that depends logarithmically on Pe. Thus the channel cross section is rapidly mixed, and Taylor dispersion along the channel is significantly reduced. Reprinted with permission from Stroock, Dertinger, Ajdari, et al., 2002. (C2002, AAAS.

fluid elements. After $N$ cycles requiring $\tau_{\text {cyc }} \sim N L_{\text {cyc }} / U$, where $L_{\text {cyc }}$ is the cycle length, stripes are separated by a distance $h_{\mathrm{eff}} \sim h / 2^{N}$. Following the above reasoning, mixing occurs when the time to diffuse between stripes $h_{\text {eff }}^{2} / D$ is comparable to the cycle time $\tau_{\text {cyc }}$, which requires

$$
\begin{aligned}
N_{\text {chaotic }} & \sim \ln \mathrm{Pe}, \\
\tau_{\text {chaotic }} & \sim \frac{L_{\text {cyc }}}{h} \frac{\ln \mathrm{Pe}}{\mathrm{Pe}} \tau_{D} .
\end{aligned}
$$

Indeed, the number of cycles (or mixing length) measured in the staggered herringbone mixer depends logarithmically on Pe over six decades (Stroock, Dertinger, Ajdari, et al., 2002).

Another interesting fact about chaotic mixing in such continuous-flow systems is that Taylor dispersion is dramatically reduced (Stroock, Dertinger, Ajdari, et al., 2002). Taylor dispersion, as described above, cuts off convective stretching when fluid elements have sampled all cross-sectional positions and, therefore, all axial velocities. In the standard picture of Taylor dispersion, this "sampling" occurs due to diffusion alone. In the chaotic mixer described above, however, tracers explore the channel cross section chaotically, quickly cutting off convective spreading and dramatically reducing axial dispersion. Such dispersion reduction was studied in the twisted pipe (Jones and Young, 1994; Castelain et al., 2000; Rush et al., 2002) and measured in the herringbone mixer (Stroock, Dertinger, Ajdari, et al., 2002).

Steady, incompressible two-dimensional flows are integrable and cannot exhibit chaotic trajectories [Ottino 
(1989), Aref (2002), Wiggins and Ottino (2004), and references therein]. However, steady three-dimensional flows can have chaotic streamlines (Bajer and Moffatt, 1990; Stone et al., 1991; Kroujiline and Stone, 1999; Ajdari, Stroock, Dertingeret al., 2002; Stroock, Dertinger, Whitesides, et al., 2002), as can unsteady twodimensional flows (Aref, 1984; Ottino, 1989). "Topologic" chaotic mixing uses interlocking helical channels designed to replicate the chaotic Baker's transformation (Chen and Meiners, 2004). Oscillatory flows driven across a main channel stretch and fold fluid in the primary flow into itself, significantly enhancing mixing (Glasgow and Aubry, 2003; Bottausci et al., 2004; Okkels and Tabeling, 2004; Tabeling et al., 2004). Chaotic flows often contain ordered regions that hamper mixing. The barriers they present can be overcome by driving singularity-induced diffusion to achieve uniform mixing in time-dependent, three-dimensional laminar flows (Solomon and Mezic, 2003).

The above ideas employed continuous chaotic flows. Bounded Stokes flows may also be chaotic, since their streamlines need not be closed (Bajer and Moffatt, 1990; Stone et al., 1991; Kroujiline and Stone, 1999). This allows chaotic mixing to occur within drops, where a typical technique involves superposing two simple flow fields. A sedimenting drop in a shear flow (Bryden and Brenner, 1999), a drop driven along a turning microchannel (Song, Bringer, et al., 2003; Song and Ismagilov, 2003; Song, Tice, et al., 2003; Tice et al., 2003; Bringer et al., 2004), and oscillating drops (Angilella and Brancher, 2003) all can exhibit chaotic mixing. An applied electric field induces an electrohydrodynamic quadrupolar flow in the droplet (Taylor, 1966; Melcher and Taylor, 1969; Torza et al., 1971) that, when combined with the dipolar flow induced by drop sedimentation, can be chaotic (Lee et al., 2000; Ward and Homsy, 2001, 2003; Im and Kang, 2003).

In principle, any technique for manipulating fluid flow can be used to promote rapid mixing. Examples include electrowetting (Paik et al., 2003) nonlinear electrokinetic effects (Takhistov et al., 2003; Bazant and Squires, 2004), electro-osmosis in inhomogeneously charged channels (Qian and Bau, 2002), acoustic streaming (Yang et al., 2000, 2001), and others.

Lastly, a few microfluidic mixers have been developed by direct analogy with turbulence. Rather than employ a deterministic but chaotic flow, these micromixers destabilize the flow using noninertial means. Electrohydrodynamic instabilities (Oddy et al., 2001; El Moctar et al., 2003; Lin et al., 2004; Fig. 36) and elastic instabilities of dilute polymer solutions (Groisman and Steinberg, 2001, 2004) have proven effective.

\section{The capillary number: Free-surface deformations}

Thus far, we have assumed that fluids are miscible: parallel streams were assumed to flow alongside each other, and tracers diffused freely from one stream to the other. Between immiscible fluids, however, a surface tension $\gamma$ affects the dynamics of the free surface. For ex-

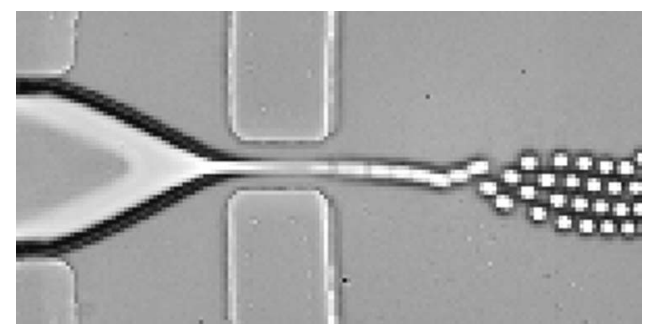

FIG. 14. Capillary instabilities in a microfluidic two-phase flow. A stream of water flows between streams of oil and is geometrically focused into a narrow cylindrical jet. The jet is destabilized by the Rayleigh-plateau instability and forms small, monodisperse droplets. Reprinted with permission from Anna et al., 2003. (C2003, AIP.

ample, Fig. 14 (Anna et al., 2003) shows a thin central stream of water breaking into drops due to the Rayleigh-Plateau instability (Rayleigh, 1879; Chandrasekhar, 1981; de Gennes, Brouchard-Wyart, and Quere, 2004). Clearly, surface tension can play an important role in microfluidic flows when immiscible free surfaces are present.

\section{Droplet formation in two-phase flows}

Thorsen et al. (2001) demonstrated that microfluidic devices could be used to create controllable droplet emulsions in immiscible fluids, by injecting water into a stream of oil at a $\mathrm{T}$ junction (Fig. 15). Were there no interfacial tension between water and oil, the two streams would flow alongside one other, as in the T sensors. However, competing stresses drive the interface: surface tension acts to reduce the interfacial area, and viscous stresses act to extend and drag the interface downstream. These stresses destabilize the interface and cause droplets of radius $R$ to form (Rallison, 1984; Stone, 1994). An estimate for the size of the droplets can be obtained by balancing the two stresses on the inter-

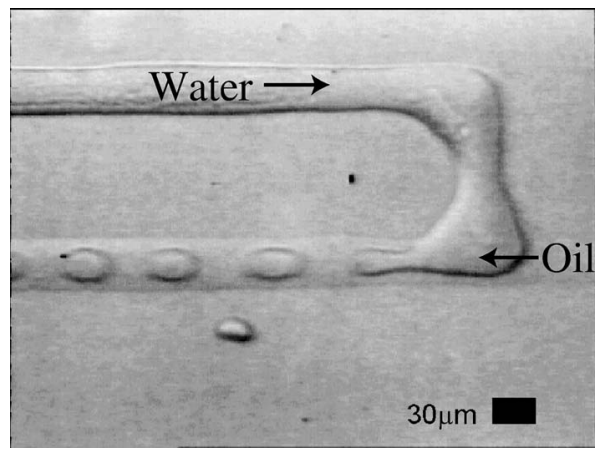

FIG. 15. Monodisperse microdroplet generation in a simple microfluidic device. The interface between flowing immiscible fluids is driven by competing stresses: viscous shear stresses tend to extend and drag the interface, whereas surface tension tends to reduce the interfacial area. The competition between the two leads to drop sizes (scaled by the channel height $h$ ) of order $R \sim \mathrm{Ca}^{-1}$. Adapted with permission from Thorsen et al., 2001. 


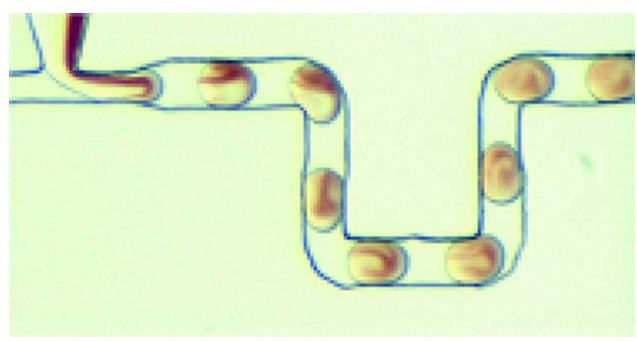

FIG. 16. (Color in online edition) Chaotic advection in a pumped droplet. A droplet driven down a channel experiences a dipolar, circulating flow; when the channel changes direction, the axis of the dipole changes as well. Alternating dipolar flows drive rapid mixing by chaotic advection, with mixing lengths that vary logarithmically with Pe. Reprinted with permission from Song, Bringer, et al., 2003. (C2003, AIP.

face (Taylor, 1934). Capillary stresses of magnitude $\gamma / R$ balance viscous stresses $\eta U_{0} / h$, giving a characteristic droplet size

$$
R \sim \frac{\gamma}{\eta U_{0}} h=\frac{h}{\mathrm{Ca}} .
$$

Here we have introduced the capillary number

$$
\mathrm{Ca}=\frac{\eta U_{0}}{\gamma},
$$

a dimensionless parameter found whenever interfacial stresses compete with viscous stresses.

An advantage to this strategy is that one can produce monodisperse droplets, due to the deterministic nature of microfluidic flows. Further work on droplet-forming devices includes studies of the rich variety of droplet patterns that forms in channels (Thorsen et al., 2001; Dreyfus et al., 2003), and studies of microchannel emulsification (Sugiura et al., 2000, 2001, 2002a, 2002b). Smaller droplets can be formed using flow focusing, either by increasing shear gradients or by drawing the stream into a thin jet that breaks up by the RayleighPlateau instability (Fig. 14; Anna et al., 2003). Notably, the dynamics of flow-focusing bubble-forming systems is largely independent of $\mathrm{Ca}$ for both low-Re (Garstecki et al., 2004) and high-Re (Ganan-Calvo, 1998; GananCalvo and Gordillo, 2001; Gordillo et al., 2001) flows. Geometric techniques have been used to create droplets of varying sizes and size distributions (Link et al., 2004). Introducing colloidal spheres allows the production of monodisperse tetrahedral photonic balls for potential use as building blocks for photonic materials (Yi, Jeon, et al., 2003; Yi, Thorsen, et al., 2003).

This strategy has been used to study chemical reaction kinetics on millisecond time scales by injecting reactive chemicals together to form drops (Song, Bringer, et al., 2003; Song and Ismagilov, 2003; Song, Tice, et al., 2003; Tice et al., 2003; Bringer et al., 2004), and in developing a platform for protein crystallization (Zheng et al., 2003). Droplets are advantageous for this sort of study because reagents are not dispersed beyond the boundary of the drop. Figure 16 depicts mixing in droplets pumped down a curving microchannel-as with the chaotic mixers described in Sec. II.B.3, the mixing time for these droplets was measured to depend logarithmically on Pe (Song, Bringer, et al., 2003).

\section{Fluid control using patterned surfaces}

The large surface-to-volume ratios of microfluidic devices render surface effects increasingly important, particularly when free fluid surfaces are present. Surface tensions can exert significant stress that results in free surface deformations and/or bulk liquid motion.

Most simply, capillary forces tend to draw fluid into wetting microchannels wherein the solid-liquid interfacial energy $\gamma_{s l}$ is lower than for the solid-gas interface $\gamma_{s g}$. (Note that we consider the second fluid to be a gas for simplicity of notation; it could also be a second liquid.) The dynamics of surface tension-driven fluid intrusion into a pipe of radius $w$ follow from a balance of capillary and viscous forces (Washburn, 1921). The curved meniscus at the fluid-gas interface gives rise to a Laplace pressure $\Delta p \sim \Delta \gamma / w$, where $\Delta \gamma=\gamma_{s l}-\gamma_{s g}$ represents the net energy (per area) gained or lost as the fluid interface advances. This pressure drives the fluid column of length $z$ into the channel via Poiseuille flow with velocity scale

$$
u \sim \frac{\Delta p w^{2}}{\eta z} \sim \frac{\Delta \gamma w}{\eta z} .
$$

As expected, the capillary number determines the dynamics $(\mathrm{Ca} \sim w / z)$. Because the column length $z$ changes as the front moves $(u=\dot{z})$, fluid invades at an ever slowing rate, according to the Washburn equation

$$
z \sim\left(\frac{\Delta \gamma w}{\eta} t\right)^{1 / 2}
$$

One subtle point about wetting-driven scaling is in order. Usually, the dynamics of such systems are found by assuming that the energy gained by wetting is dissipated viscously-presumably in the bulk of the fluid. However, fluid dissipation diverges near the contact line, requiring a cutoff length scale from microscopic physics (Dussan, 1979; de Gennes, 1985; de Gennes, Brochard-Wyart, and Quere, 2004; Eggers and Stone, 2004). Depending on the amount of flowing fluid, either bulk or contact line dissipation can determine the dynamics. In this review, we will always assume bulk dissipation to dominate, although this need not be the case.

Drops can be repeatedly drawn into a capillary pump consisting of multiple, parallel, wettable channels, where a narrow capillary retention valve brakes the motion (Juncker et al., 2002). Handique et al. (1997) use nonwetting channel walls to control capillary motion-for example, a nonwetting section arrests capillary intrusion. Furthermore, fluid does not enter nonwetting side channels, which have thus been used as vents for droplets driven by pressurized gas-once the droplet moves past the vent, the gas escapes through the side channel rather than continue to drive the droplet. Last, gas forced through nonwetting side channels have been used to 

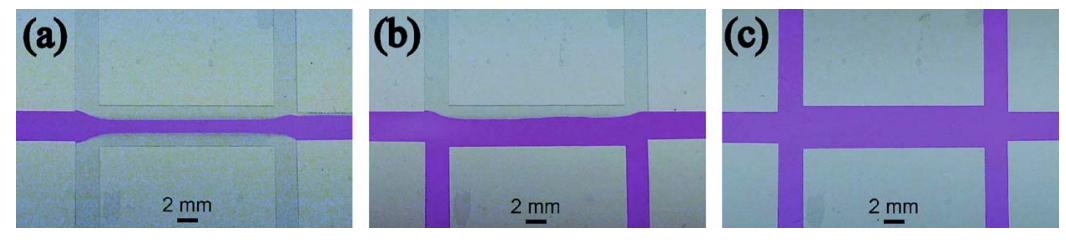

FIG. 17. (Color in online edition) Pressure-sensitive valves based on patterned surfaces with differing wetting properties. The central stripe is hydrophilic, the lower channel is moderately hydrophobic, and the upper channel is most hydrophobic. (a) Virtual walls contain the fluid so long as the applied pressure is below a critical value, and water is pumped along the central stripe. (b) When the applied pressure exceeds a threshold value, however, the fluid is driven through one virtual wall and onto the moderately hydrophobic surface. (c) Finally, above a second threshold, the fluid is driven onto the upper (most hydrophobic) surface, and flows into all channels. Reprinted with permission from Zhao et al., 2001. (C2001, AAAS.

split and meter droplets at precise locations. This metered injection system has been used, for example, in an integrated DNA analysis device (Burns et al., 1998).

Hydrophobic stripes have been used to confine fluids (Handique et al., 1997), and to create "wall-less" channels (Gau et al., 1999; Kataoka and Troian, 1999; Zhao et al., 2001). The static structures of such confined liquids has been studied (Gau et al., 1999; Darhuber et al., 2000), and capillary spreading along hydrophilic stripes has been shown theoretically and experimentally to vary with the square root of time (Darhuber et al., 2001). Spreading along a hydrophilic stripe of width $w$ resembles Washburn's analysis, except that the height of the fluid stripe varies as $h \sim w^{2} / R_{r}$, where $R_{r}$ is the radius of curvature of the inlet reservoir drop, leading to $z^{2} \sim\left(\gamma w^{4} / \eta R_{r}^{3}\right) t$ (Darhuber et al., 2001). Spreading along capillary microstripes must occur slowly enough that $\mathrm{Ca} \ll 1$; otherwise, capillary forces are not strong enough to keep the fluid confined to the hydrophilic stripe.

Patterned surfaces have been employed in closed microchannels to manipulate multiple immiscible fluids within a single microchannel. Fluids can be driven simply using an applied pressure low enough that capillary forces keep the interface pinned to the edge of the patterned stripe (that is, $\Delta p \lesssim \gamma / w$ ) (Zhao, Moore, and Beebe, 2002; Zhao, Viernes, et al., 2002). If the fluid exceeds this critical pressure, it is forced onto the hydrophobic region. This effect forms the basis for the pressure-sensitive gates in Fig. 17 (Zhao et al., 2001, 2003), in which liquid expands into nonwetting channels once a critical pressure has been exceeded. With multiple fluids in the channel, reaction kinetics can be probed at their large shared interface (Zhao et al., 2001), and immiscible streams can be driven in opposite directions to enable highly efficient countercurrent exchange of tracers like gas molecules or heat across the interface (Zhao, Viernes, et al., 2002).

\section{Fluid manipulation with capillary forces}

Capillary forces can also be used to manipulate and transport fluids with free surfaces. In equilibrium, free surfaces are determined by a balance of interfacial forces. Actively modifying interfacial energies, however, disrupts the balance and causes motion.

Broadly speaking, capillary manipulation of microfluids has been achieved via two strategies: either by modi- fying the solid-liquid surface tension $\gamma_{s l}$, or by inducing a gradient in the liquid-gas surface tension $\gamma$. Examples of the former include flows due to electrowetting, surface gradients, and reactive flows. The latter include thermocapillary, electrocapillary, and solutocapillary motion.

\section{a. Solid-liquid interfacial energy manipulation}

Several strategies for exploiting solid-liquid surface tension have been demonstrated for microfluidic manipulation. See Bain (2001) for a brief review. Figure 18 presents one of the simplest examples: a long droplet of length $L$ in a channel of radius $w$ with an inhomogeneous surface: hydrophobic (with $\gamma_{s l}^{L}$ ) for $z<0$ and hydrophilic $\left(\gamma_{s l}^{R}>\gamma_{s l}^{L}\right)$ for $z>0$ (Raphael, 1988; Ondarçuhu and Veyssie, 1991; Weislogel, 1997). Energetically, the droplet wants to move onto the hydrophilic surface, and moving with velocity $U$ decreases the stored interfacial energy at a rate $\sim \Delta \gamma w U$, where $\Delta \gamma=\gamma_{s l}^{L}-\gamma_{s l}^{R}$. This energy is lost to viscous dissipation, which consumes a power $\eta \int(\partial u / \partial z)^{2} d V \sim \eta U^{2} L$ when dissipation is dominated by viscous shear in the bulk. Assuming the capillary energy released to be balanced by viscous dissipation gives

$$
\frac{\eta U}{\Delta \gamma} \equiv \mathrm{Ca}=\frac{w}{L} .
$$

Again we see that the capillary number arises naturally, because capillary stresses are balanced by viscous

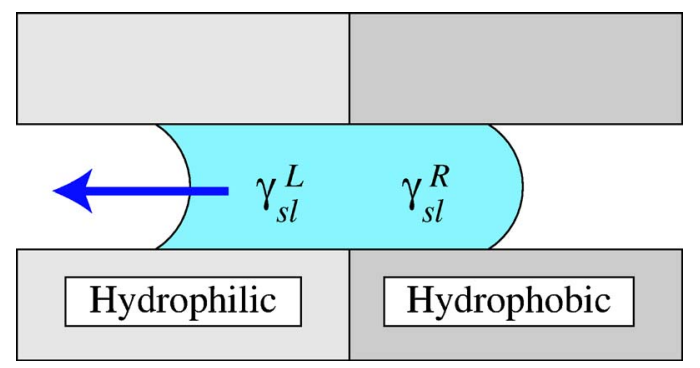

FIG. 18. (Color in online edition) Droplet motion due to a gradient in solid-liquid interfacial energy. A droplet straddling hydrophobic and hydrophilic surfaces can release stored interfacial energy by moving further into the hydrophilic region. A droplet moving with velocity $U$ releases $\Delta \gamma w U$ energy per unit time; this energy goes into viscous dissipation, which consumes a power $\eta U^{2} L$. As a result, the droplet moves with velocity $U \sim \Delta \gamma w /(\eta L)$. 
stresses. In this case, the droplet moves with velocity scale $U \sim \Delta \gamma w / \eta L$.

Below, we discuss specific realizations: surfaces with static wettability gradients, thermal gradients, reactive fluids, self-propelling bi-slugs, optowetting, and (most popular) electrowetting. Although the examples we discuss below may employ alternate geometries, the basic physics is the same for droplets moving through a channel, two-dimensional (2D)/3D droplets moving on a substrate, or $2 \mathrm{D} / 3 \mathrm{D}$ drops moving between two plates. In principle, all can be used in microfluidic systems.

\section{b. Surfaces with wettability gradients}

The motion of a small fluid droplet on a surface with a wettability (interfacial energy) gradient was originally examined theoretically as part of a study of cell spreading (Greenspan, 1978). Further treatments followed, including fluid spreading on a patchy surface (Raphael, 1988). Brochard (1989) provided an elegant and comprehensive study of the motion of large or small droplets [compared with the capillary length $(\gamma / \rho g)^{1 / 2}$ ] due to both solid surfaces with wettability gradients, and for liquid-liquid tension gradients. Experimentally, fluids straddling a wetting discontinuity were observed to move to the more highly wetting side (Ondarçuhu and Veyssie, 1991; Weislogel, 1997), and a fluid droplet was shown to travel uphill on a surface with wettability gradient (Chaudhury and Whitesides, 1992). A more recent manifestation involves the rapid motion of water droplets condensing on a surface with a static wettability gradient. In this case, condensation energy establishes a thermal gradient, further driving motion (Daniel et al., 2001; Wasan et al., 2001).

\section{i. Thermal gradients [Fig. 19(a)]}

While more attention has been paid to temperature variations in fluid-fluid surface tensions, and the Marangoni flows that result, the solid-liquid interfacial tension depends on temperature as well. A slug of fluid in a microchannel moves to reduce its total interfacial energy, as does a droplet on a solid surface with a thermal gradient. The latter, however, can also move due to Marangoni stresses that drive the interface itself (Brochard, 1989; Brzoska et al., 1993). Burns et al. employ these effects in DNA analysis devices (Burns et al., 1996, 1998; Sammarco and Burns, 1999, 2000), although their term, "thermocapillary," has traditionally referred to interfacial motion due to surface tension gradients (Young et $a l ., 1959)$. By analogy with electrowetting, perhaps thermowetting would be more appropriate.

\section{ii. Reactive fluids [Fig. 19(b)]}

The above examples involve constant, but inhomogeneous, interfacial energies $\gamma_{s l}$. Another technique for driving fluid motion involves reactive fluids: a droplet that contains a chemical that decreases the wettability of a solid surface spontaneously moves, seeking to avoid any areas it has visited (Bain et al., 1994; Dos Santos and Ondarçuhu, 1995; Shanahan and de Gennes, 1997; de (a)

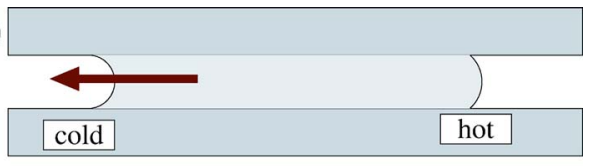

(b)

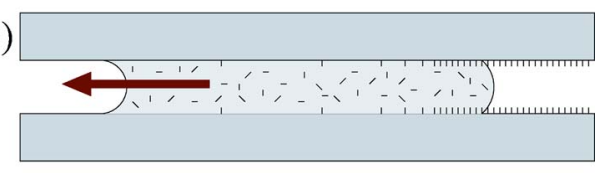

(c)

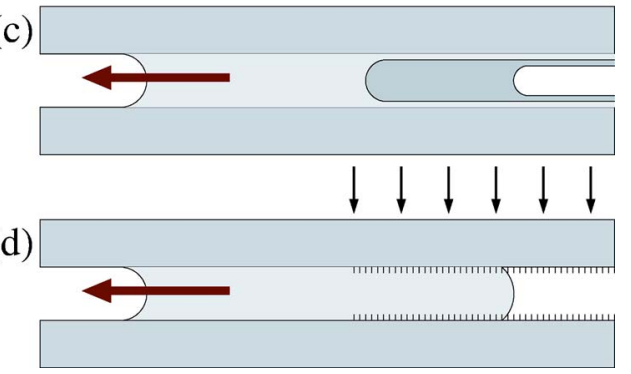

FIG. 19. (Color in online edition) Driving droplet motion with gradients in solid-liquid interfacial energy driven by (a) thermal gradients, (b) droplets that contain a chemical that reacts to decrease surface wettability, (c) liquid bi-slugs that leave a coating film that lowers the overall surface energy, and (d) light-induced reactions that create wettability gradients.

Gennes, 1998; Lee and Laibinis, 2000; Lee, Kwok, and Laibinis 2002). Such techniques have not yet been used explicitly for microfluidics, but could be useful for oneshot applications.

\section{iii. Self-propelling liquid bi-slugs [Fig. 19(c)]}

A compound liquid bi-slug consisting of two individual slugs of different liquids in a capillary can undergo a capillary-driven self-propelled motion (Bico and Quere, 2000, 2002). Moving the bi-slug creates interfacial area behind the slug, and destroys it in front. Similar to the reactive fluids described above (Bain et al., 1994; Dos Santos and Ondarçuhu, 1995), this surface energy alteration provides a driving force for droplet motion. The dependence of bi-slug velocity upon wetting properties, viscosity differences, and bi-slug length has been explored theoretically and experimentally (Bico and Quere, 2002). Like reactive fluids, bi-slugs alter the surface they have visited and stop after exhausting their driving fuel.

\section{iv. Optowetting [Fig. 19(d)]}

Light can drive droplet motion when the surface is coated with a photosensitive material. Static optowetting, i.e., a change in contact angle change due to uniform light irradiation, has been reported (Wang et al., 1997; Abbott et al., 1999; Shin and Abbott, 1999; Miyauchi et al., 2002; Huang et al., 2003). Furthermore, droplet motion has been driven using gradients in light intensity (Ichimura et al., 2000; Oh et al., 2002). UV light of varying intensity causes a wettability gradient that drives 


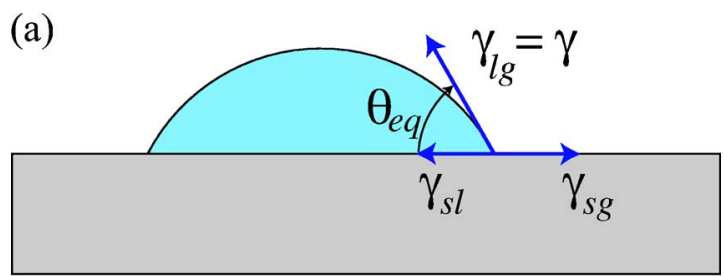

(b)

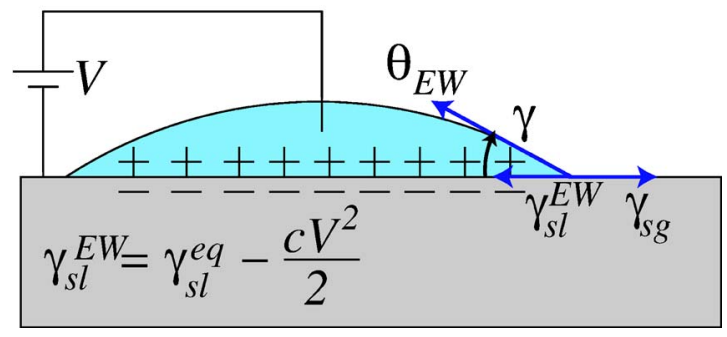

FIG. 20. (Color in online edition) Wetting and electrowetting. (a) In standard equilibrium wetting, each interface has an associated tension, and the sum of forces on the three-phase contact line must balance, leading to an equilibrium contact angle $\theta_{\text {eq. }}$ (b) When a potential difference $V$ is applied between the drop and the solid surface it touches, an effective parallelplate capacitor $c$ is charged at the interface. The charged capacitor lowers the solid-liquid interfacial energy by an amount $c V^{2} / 2$, which lowers the steady-state contact angle $\theta_{\mathrm{EW}}$.

droplet motion, which can be reversed by blue-light irradiation. Velocities of $36 \mu \mathrm{m} / \mathrm{s}$ were reported for $2-\mu \mathrm{l}$ olive oil drops.

\section{v. Electrowetting}

Finally, we explore electrowetting, which is probably the most popular means of driving microfluidic motion by modifying $\gamma_{s l}$ (Quilliet and Berge, 2001). The basic physics of electrowetting can be understood within the picture of equilibrium wetting [Fig. 20(a)]. For reviews, see Dussan (1979), de Gennes (1985), de Gennes, Brochard-Wyart, and Quere (2004), and Eggers and Stone (2004). A tension (energy/area, or force/length) is associated with each interface: solid-liquid $\gamma_{s l}$, solid-gas $\gamma_{s g}$, liquid-gas $\gamma_{l g} \equiv \gamma$. Each exerts a force on the threephase contact line, and a balance of forces gives Young's equation,

$$
\gamma_{s g}-\gamma_{s l}-\gamma \cos \theta_{\mathrm{eq}}=0,
$$

defining the equilibrium contact angle.

When a potential difference $V$ is applied between the drop and the substrate, the solid-liquid interface behaves as a capacitor, with capacitance per unit area $c$ [Fig. 20(b)]. The larger the solid-liquid interface, the greater the total capacitance, which decreases the total electrostatic energy and drives the drop to spread. The free energy per unit interfacial area is lowered by an amount $c V^{2} / 2$, since $c V^{2} / 2$ is stored in the capacitor and $-c V^{2}$ is done by the battery (Feynman et al., 1989). Moving the contact line a distance $\Delta z$ requires a virtual work (per unit length) $-c V^{2} \Delta z / 2$, so that a force (per unit length) $c V^{2} / 2$ pulls the line out. This electrowetting tension gives a new force balance,

$$
\gamma_{s g}-\left(\gamma_{s l}-\frac{c V^{2}}{2}\right)-\gamma \cos \theta_{\mathrm{EW}}=0,
$$

and the contact angle changes according to the Lippmann equation (Lippmann, 1875)

$$
\cos \theta_{\mathrm{EW}}=\cos \theta_{\mathrm{eq}}+\frac{c V^{2}}{2 \gamma} \text {. }
$$

Similar physics occurs at electrified fluid-fluid interfaces, giving electrocapillarity.

Initially, electrowetting was performed on bare electrodes, in which case the capacitance of the waterelectrode surface is given by the capacitance of the ionic double layer within the water, $c \sim \epsilon_{w} / \lambda_{D}$, where $\lambda_{D}$ is the Debye screening length, typically of order nanometers (Sec. III.A). The large capacitance of the double layer allows large contact angle changes for relatively small applied voltages. However, electrochemical reactions occur at electrode-water interfaces when the applied potential exceeds a critical (system-dependent) value typically of order $1 \mathrm{~V}$. Such reactions fundamentally alter the physics of the system, e.g., by creating gaseous oxygen and hydrogen, by injecting metallic ions into solution, or by changing the solution $p \mathrm{H}$.

More recently, electrowetting using electrodes coated with a thin dielectric layer, called EWOD (electrowetting on dielectric) or EICE (electrowetting on insulator coated electrodes), has been probed (Pollack et al., 2000; Quilliet and Berge, 2001; Saeki et al., 2001; Seyrat and Hayes, 2001; Lee, Moon, et al., 2002; Moon et al., 2002). Contact angle changes of $40^{\circ}$ can be driven with as low as 15-V applied potentials (Moon et al., 2002). Several advantages stand out: (i) higher potentials can be applied without causing undesired electrochemical reactions; (ii) clean surfaces can be prepared, reducing surface defects that pin the contact line and reduce reversibility; and (iii) highly nonwetting surfaces can be made that enhance the changes due to electrowetting. Drawbacks include (i) lower capacitance requires a stronger applied potential to achieve a given contact angle change, and (ii) biomolecules tend to bind nonspecifically to the hydrophobic surfaces typically used in such studies (Yoon and Garrell, 2003).

A common observation is that the contact angle increases according to Eq. (22) until some critical voltage $V_{c}$, at which point electrowetting saturates. [Recently, however, Krupenkin et al. (2004) demonstrated that a superhydrophobic nanostructured surface becomes completely wet with only $22 \mathrm{~V}$.] More generally, the saturation mechanism remains unknown (Quilliet and Berge, 2001; Moon et al., 2002), although various mechanisms have been proposed: vanishing $\gamma_{s l}$ for potentials exceeding a critical value (Peykov et al., 2000), ionization near the wedge-shaped contact line (Vallet et al., 1999), charge trapping in the dielectric layer (Verheijen and Prins, 1999), charge injection into a polymeric layer 


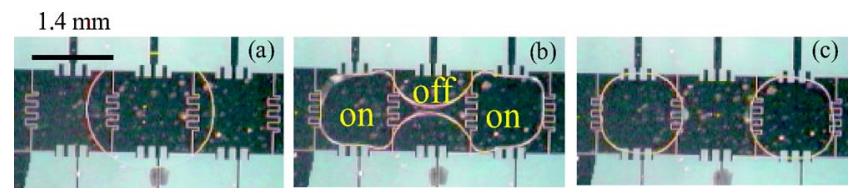

FIG. 21. (Color in online edition) A three-electrode system splits a 200-nl drop of deionized water in 8/30 s using electrowetting. Scale bar is $1.4 \mathrm{~mm}$. Reprinted with permission from Cho, Moon, and Kim, 2003. (C2003, IEEE.

(Janocha et al., 2000), dielectric breakdown (Blake et al., 2000; Seyrat and Hayes, 2001), surface roughness (Janocha et al., 2000), water molecule adsorption (Schneemilch et al., 2000), and vertical forces on the contact line (Kang, 2002).

A clear advantage to electrocapillary or electrowetting is the speed with which potentials can be applied (e.g., compared to thermal transport). For example, an electrowetting display was demonstrated to have $12-\mathrm{ms}$ switching times, which is fast enough for video (Hayes and Feenstra, 2003), and a fluid column was shown to rise $20 \mathrm{~mm}$ over about $1 \mathrm{~s}$ (Welters and Fokkink, 1998). Droplet manipulation is likewise fast-velocities on solid substrates of order $1 \mathrm{~mm} / \mathrm{s}-10 \mathrm{~cm} / \mathrm{s}$ have been demonstrated (Washizu, 1998; Pollack et al., 2000, 2002; Cho, Moon, and Kim, 2003) and drops have been split in less than a second (Cho, Moon, and Kim, 2003) (Fig. 21).

Early work suggesting displays based on electrowetting (Beni and Hackwood, 1981; Beni and Tenan, 1981), has progessed to video-speed "electronic paper" (Hayes and Feenstra, 2003; Roques-Carmes et al., 2004). Microdroplets act as optical lenses, for which electrowetting allows precise alignment and focal-length tunability (Berge and Peseux, 2000; Krupenkin et al., 2003; Yang et al., 2003). Furthermore, dynamically tunable optical fiber devices, switches, and waveguides have been developed based on electrowetting (Jackel et al., 1982, 1983; Mach et al., 2002; Acharya et al., 2003; Hsieh et al., 2003). Specific microfluidic examples include electrowettingbased micropumps (Colgate and Matsumoto, 1990; Jun and Kim, 1998; Yun et al., 2002), creation, manipulation, and metering of fluid droplets (Lee and Kim, 2000; Pollack et al., 2000; Lee, Moon, et al., 2002; Cho, Moon, and Kim, 2003; Kuo et al., 2003; Ren et al., 2004), and chemical reactors that merge and mix droplets (presumably) by electrowetting (Taniguchi et al., 2002). Electrowetting in multiple parallel channels gives rise to an electrocapillary pressure that can be used to drive fluid motion (Prins et al., 2001), and "designer" droplet shapes can be manipulated and maintained (Mugele and Herminghaus, 2002; Bienia et al., 2003). In biofluidic chips, biomolecules have a tendency to passively adsorb onto hydrophobic surfaces (Absolom et al., 1987; van der Vegt et al., 1996; Makohliso et al., 1998), reducing reversibility in electrowetting devices. Voltage strategies have been developed to reduce this surface fouling (Yoon and Garrell, 2003). Finally, electrowetting increases the loading efficiency of biological microcantilever arrays (Belaubre et al., 2003).
Several variants on the theme of electrowetting have been demonstrated. Surfaces have been developed that, under a sufficiently strong applied potential, undergo an electrochemical reaction that abruptly changes the contact angle (Sondag-Huethorst and Fokkink, 1994). Similarly, monolayers have been demonstrated to have electrically driven conformational changes, allowing reversible wettability control (Lahann et al., 2003). Last, optoelectrowetting employs a photoconducting dielectric layer that switches from dielectric to conductor when illuminated. In this way, light triggers an enhancement of electrowetting by effectively reducing the parallel plate capacitor separation (Chiou et al., 2003).

\section{vi. Dielectrophoresis}

Finally, we briefly discuss dielectrophoresis, in which a dielectric body is forced either up or down a gradient in electric field strength in order to minimize total electrostatic energy (Pohl, 1978). This phenomenon is perhaps more familiar to physicists from optical tweezers, which represents a higher-frequency (optical) regime. Because the dielectric constant of a body is frequency dependent, particles can be separated and manipulated using ac fields of various frequencies [reviewed by Pohl (1978); Green, Ramos, and Morgan (2000); Gascoyne and Vykoukal (2004)], and drive droplet-based microanalysis platforms (Jones et al., 2001; Gascoyne et al., 2002; Gascoyne and Vykoukal, 2004; Schwartz et al., 2004). Although droplet dielectrophoresis and electrowetting are often discussed together, the two represent distinct physical mechanisms [see, e.g., Jones (2002) and Jones et al. (2003)].

\section{c. Liquid-liquid surface tension manipulation}

The above examples involved a droplet moving down a gradient in total interfacial energy, with a speed set dynamically - the energy released as the droplet moved was lost to viscous dissipation. Here, we discuss a final, more subtle capillary phenomenon. We consider microfluidic motions established by the motion of fluid-fluid interfaces, which move in response to unbalanced stresses. A fluid-fluid interfacial tension depends upon temperature, electrostatic potential, and surfactant concentration, and surface tension gradients can be established by externally inducing a gradient in such properties. Gradients in interfacial tension along a free surface set the interface itself into motion, giving a fluid flow generally known as Marangoni flow.

Droplets suspended in a fluid with a background gradient of temperature, electric potential, or surfactant concentration will experience thermocapillary (Young et al., 1959; Chabbra and De Kee, 1992), electrocapillary (Newman, 1991), or solutocapillary (Levich, 1962; Levich and Kuznetsov, 1962; Levich and Krylov, 1969) motion (Fig. 22). Below, we discuss microfluidic applications of these free-surface flows. As above, viscous stresses balance capillary stresses, and thus their motion is set by the capillary number $\mathrm{Ca} \sim 1$, or $U \sim \Delta \gamma / \eta$. For thermocapillary motion, $\Delta \gamma \sim(d \gamma / d T)(\nabla T) R$, for solutocap- 
(a)

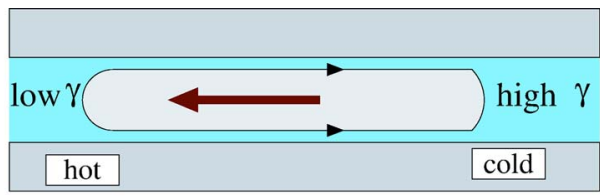

(b)

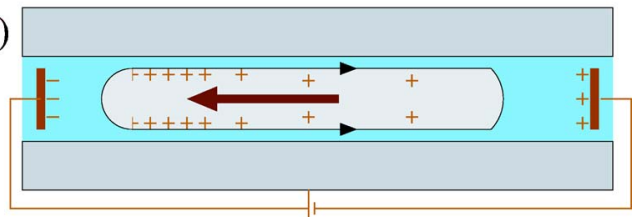

V

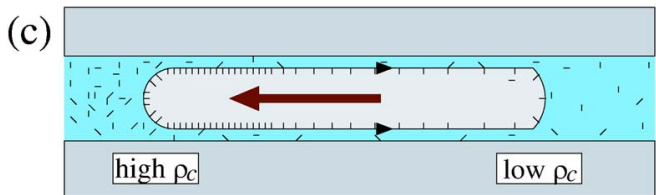

FIG. 22. (Color in online edition) Thermocapillary, electrocapillary, and solutocapillary droplet motion occurs due to surface tension gradients arising from (a) thermal gradients in the background solution that drive droplets to warmer temperatures; (b) electric fields that drive droplets away from the similarly charged electrode; and (c) background surfactant gradients that lead droplets to move towards regions of greater surfactant concentration.

illary motion, $\Delta \gamma \sim\left(d \gamma / d \rho_{c}\right)\left(\nabla \rho_{c}\right) R$, and for electrocapillary motion, $\Delta \gamma \sim(d \gamma / d V)(\nabla V) R \sim(d \gamma / d V) q E R$, where $q$ is the interfacial charge.

Electrocapillary motion (Frumkin and Levich, 1945; Newman, 1991), and the essentially identical continuous electrowetting (Beni et al., 1982) have been little used in microfluidics other than for droplet manipulation (Lee and Kim, 2000) and in a pump design (Yun et al., 2002).

Likewise, solutocapillary flows (Levich, 1962; Levich and Kuznetsov, 1962; Levich and Krylov, 1969) have seen little use in microfluidic devices, in part because maintaining a surfactant concentration gradient is not straightforward. However, Abbott and co-workers have explored molecules that can be electrochemically switched between surfactant and nonsurfactant states. In effect, electrochemical reactions create surfactant molecules at one electrode and destroy them at the other, which set up the necessary concentration gradients to drive Marangoni flows (Gallardo et al., 1995, 1996; Bennett et al., 1996; Gallardo and Abbott, 1997). This technique can be used in closed channels by using a gel-fluid free surface rather than the fluid-air interface of open channels (Lee et al., 1999). Such electrochemical manipulation allows droplet and particle transport as well as thin-film patterning (Gallardo et al., 1999). Similar techniques involve solution-based reducing agents (Jong and Abbott, 1998) or photosensitive surfactants (Shin and Abbott, 1999) rather than electrochemical reactions. Last, electrochemically switchable surfactants have been used to selectively solubilize and deposit compounds on electrodes, providing a technique for particle separation (Rosslee and Abbott, 2001).
The most popular microfluidic Marangoni flow is thermocapillary. Homsy and co-workers completed experimental (Lajeunesse and Homsy, 2003) and theoretical (Mazouchi and Homsy, 2000, 2001) studies of thermocapillary bubble motion through capillaries which built upon two classic dynamic free-surface problems: the Bretherton problem involving the motion of a bubble through a pipe (Bretherton, 1961) and the Landau-Levich problem (Landau and Levich, 1942) involving the wetting film left on a plate pulled out of a liquid bath.

Many microfluidic thermocapillary flows occur on solid walls, where temperature variations in both $\gamma_{s l}$ and $\gamma$ drive fluidic motion (Brochard, 1989; Brzoska et al., 1993). In addition to droplet motion, thermocapillary motion has been used to drive thin liquid films to spread; classic references are Levich (1962) and Ludviksson and Lightfoot (1971). Troian and co-workers have experimentally, theoretically, and numerically explored thermocapillary microfluidic flows along patterned hydrophilic stripes (Kataoka and Troian, 1999; Darhuber, Davis, et al., 2003) and the mixing that accompanies these flows (Darhuber et al., 2004), complementing studies of thermocapillary contact line instabilities and their control (Kataoka and Troian, 1997, 1998). An optical means (via absorption) to drive thermocapillary motion was recently demonstrated, allowing feedback control methods to suppress these instabilities (Garnier et al., 2003). As with thermocapillary droplet motion, capillary forces balance viscous forces in motion along stripes, although an additional length scale arises (the droplet height $h$ depends on stripe width $w$, but may be set dynamically and thus depend on $\mathrm{Ca}$ ). Various scaling relations can be derived, and velocities up to $600 \mu \mathrm{m} / \mathrm{s}$ were observed for liquid poly(dimethysiloxane) (PDMS) drops (Darhuber, Davis, et al., 2003). High-resolution temperature distributions (Darhuber et al., 2002) have enabled thermocapillary manipulation of droplets, including droplet transport and splitting (Fig. 23; Darhuber, Valentino, et al., 2003).

Finally, we note that surface tension gradients due to thermal gradients form the basis for Marangoni-Benard convection (Davis, 1987). Recently, a ratchet pump was demonstrated by driving Marangoni-Benard convection above an asymmetrically patterned substrate (Stroock $e t$ al., 2003).

\section{The Weissenberg and Deborah numbers: Let the mountains flow}

Thus far, we have considered the influences of inertia, diffusion, and surface tension on microfluidic flows. Dissolved polymers add an elastic component to the fluid that further enriches flow behavior. Microfluidic devices have proved useful for such systems in two senses: first, to study the effects of fluid flow on polymer behavior, and second, to study the effects of polymers on the behavior of fluid flows. In both cases, microfluidic devices simplify the experiments by eliminating inertial effects that compete or mask the physics of interest. Further- 


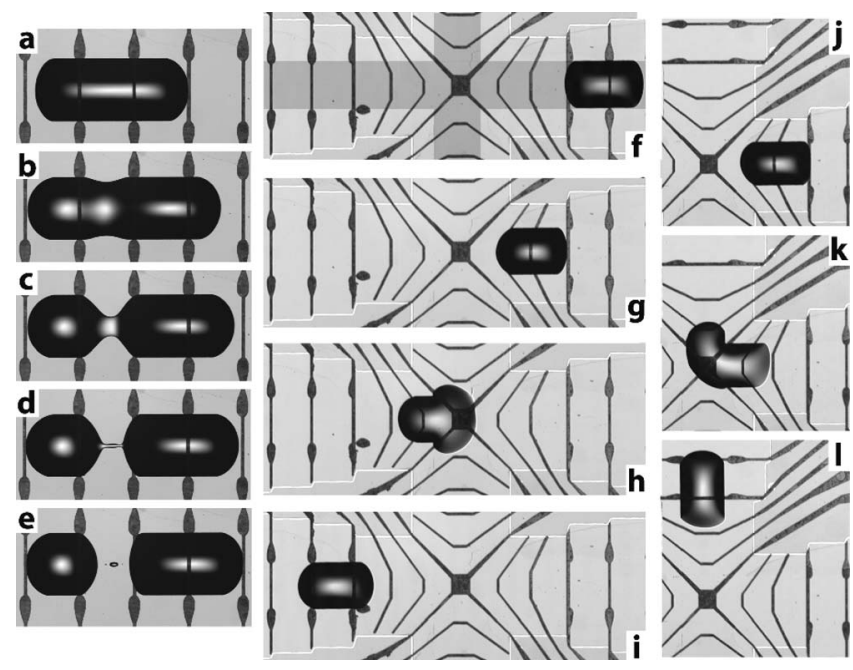

FIG. 23. Using embedded microheaters to establish thermal gradients, a dodecane drop of width $\sim 1 \mathrm{~mm}$ (a)-(e) is split in $8.5 \mathrm{~s}$, (f)-(i) travels $\sim 6 \mathrm{~mm}$ in $104 \mathrm{~s}$, and (j)-(l) turns a corner in 164 s. Reprinted with permission from Darhuber, Valentino, et al., 2003. (C2003, AIP.

more, significant shear rates can be readily obtained owing to the small dimensions of microfluidic devices, which provides a strong but controllable stretching force to distort polymer molecules and probe their elastic qualities.

First, however, we turn to a simple picture of polymer dynamics to illustrate the basic physics at hand. The simplest model system (Kuhn, 1934) treats a polymer as a dumbbell with two beads each of hydrodynamic resistance $\xi$ connected by a Hookean (entropic) spring of stiffness $k_{H}$. The spring constant $k_{H}$ can be obtained for a freely jointed chain with $N$ steps of length $b$ as $k_{H}$ $=3 k_{B} T / N b^{2}$. For simplicity, we confine the polymer to the (one-dimensional) line along the center of a contracting microchannel, as in Fig. 24. Due to the contraction, the local (extensional) flow felt by the polymer increases as it moves down the channel, roughly like $u_{z}$
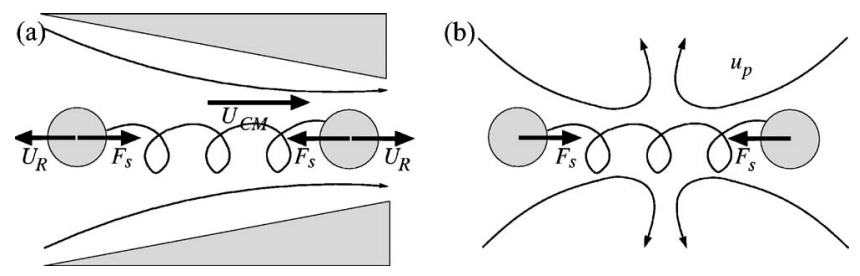

FIG. 24. The effect of a nonuniform flow on a model polymer, represented as two beads separated by a distance $R$ on a spring. (a) The center of mass of the polymer moves with velocity $U_{\mathrm{CM}}$, whereas the two beads experience relative motion $U_{R} \sim \dot{e} R_{p}-k_{H} R_{p} / \xi$ due to external flow gradients and spring forces. Brownian forces balance spring forces to give a steadystate polymer size. When $\mathrm{Wi} \gtrsim \mathcal{O}(1)$, the extensional flow overwhelms the spring, and the polymer unravels via a coil-stretch transition. (b) As the beads are pulled toward the spring, each exerts a force back on the fluid, resulting in a force-dipole flow and an anisotropic contribution to the normal stress. $=u_{0}+\dot{e} z$. The bead separation $R_{p}$ changes due to three physical effects: (i) Brownian motion drives beads apart with average speed $R_{p} \dot{R}_{p} \sim D \sim k_{B} T / \xi$, (ii) the spring pulls the beads together with velocity $\dot{R}_{p} \sim-k_{H} R_{p} / \xi$, and (iii) the extensional flow advects the forward bead more quickly than the rear bead, driving them apart with velocity $\dot{R}_{p} \sim \dot{e} R_{p}$ (Bird, Hassager, et al., 1987; Larson, 1999). Each effect has its own characteristic time scale: $\tau_{D}=R_{0}^{2} / D$ for the beads to diffusively explore a length scale $R_{0}, \tau_{p}=\xi / k_{H}$ for the polymer spring to relax, and $\tau_{e}=\dot{e}^{-1}$ is a time scale associated with the extensional flow.

While this example helps in developing physical insight and intuition for microfluidics, its applicability is generally limited. It assumes polymers do not interact, which requires the solution to be dilute. The solution stress is assumed to be dominated by the viscous solvent, rather than the polymers, constituting a so-called Boger fluid (Boger, 1977; Boger and Yeow, 1992). Polymer deformations are also assumed to be small since we have ignored the nonlinear elasticity for large deformations. Furthermore, the simplest form for the polymer-solvent interaction has been assumed, whereas in general this interaction is conformation dependent.

Despite these simplifications, several important phenomena are illustrated by the above simple example.

The Weissenberg number. In equilibrium $\dot{e}=0$, spring forces balance Brownian forces to give a characteristic polymer size $R_{0} \sim\left(k_{B} T / k_{H}\right)^{1 / 2} \sim\left(N b^{2}\right)^{1 / 2}$. By contrast, an extensional flow acts to drive the beads apart (like a negative spring), and alters the steady polymer size via

$$
R_{p}(\dot{e}) \sim\left(\frac{k_{B} T}{k_{H}-\dot{e} \xi}\right)^{1 / 2} \sim \frac{R_{0}}{(1-\mathrm{Wi})^{1 / 2}} .
$$

Here we have introduced the Weissenberg number

$$
\mathrm{Wi}=\tau_{p} \dot{e} \quad \text { or } \tau_{p} \dot{\gamma}
$$

which relates the polymer relaxation time to the flow deformation time, either inverse extension rate $\dot{e}^{-1}$ or shear rate $\dot{\gamma}^{-1}$. When $\mathrm{Wi}$ is small, the polymer relaxes before the flow deforms it significantly, and perturbations to equilibrium are small. As Wi approaches 1, the polymer does not have time to relax and is deformed significantly.

The Deborah number. Another relevant time scale $\tau_{\text {flow }}$ characteristic of the flow geometry may also exist. For example, a channel that contracts over a length $L_{0}$ introduces a geometric time scale $\tau_{\text {flow }}=L_{0} / U_{0}$ required for a polymer to traverse it. Likewise, an oscillatory flow introduces an oscillation time $\tau_{\text {flow }} \sim \omega^{-1}$. The flow time scale $\tau_{\text {flow }}$ can be long or short compared with the polymer relaxation time $\tau_{p}$, resulting in a dimensionless ratio known as the Deborah number

$$
\mathrm{De}=\tau_{p} / \tau_{\text {flow }} .
$$

Amusingly, the Deborah number is named for one of the earliest fluid mechanics, the prophetess Deborah (Reiner, 1964), who observed that “...the mountains 


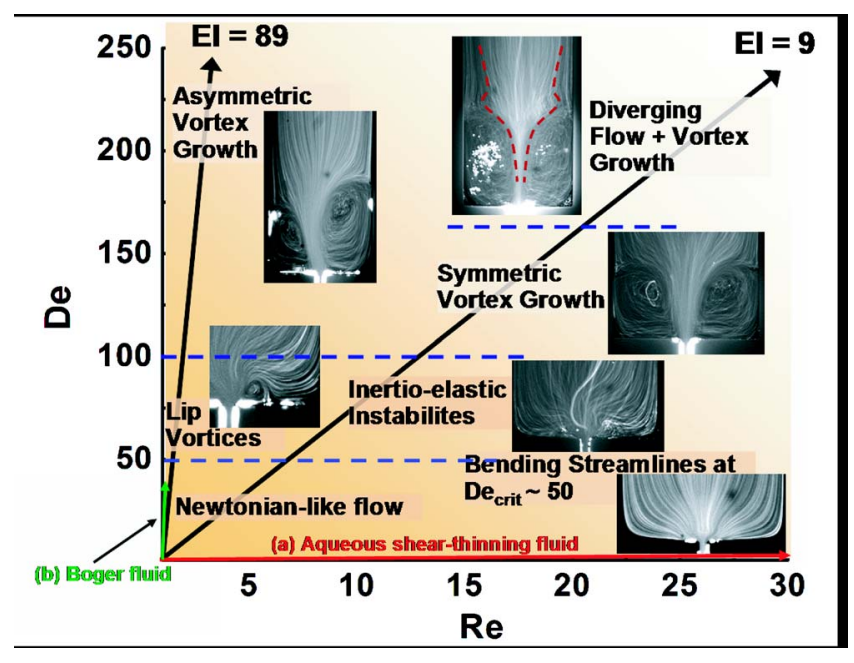

FIG. 25. (Color in online edition) Microfluidic devices allow new regimes of Re-De space to be explored for nonNewtonian flows (Rodd et al., 2004). Typical flow behaviors are shown for two different semidilute PEO solutions flowing into a 400-25- $\mu \mathrm{m}$ contraction at two different elasticity numbers $(\mathrm{El}=9$ and 89). Experiments with increasing flow rates thus correspond to straight line trajectories in Re-De space. Previous studies were limited to El extremes-highly elastic Boger fluids $(\mathrm{El} \gg 1)$ and shear-thinning viscous fluids $(\mathrm{El} \ll 1)$. Figure courtesy of L. Rodd.

flowed before the Lord..." (Judges 5:5). Implicit in her statement is the recognition that the rheological response of a material depends on the time scale over which it is observed: mountains flow on geological time scales $(\mathrm{De} \ll 1)$, but are rigid on human time scales (De $\gg 1$ ).

Note that the usage of De and Wi can vary: some references use Wi exclusively to describe steady shear flows and use De for the general case, whereas others use Wi for local flow time scales due to a local shear rate and De for global flow time scales due to a residence time in the flow.

The elasticity number. As the flow velocity $U_{0}$ increases, elastic effects become stronger and De and Wi increase. However, the Reynolds number Re increases in the same way, so that inertial effects become more important as well. The elasticity number

$$
\mathrm{El}=\frac{\mathrm{De}}{\mathrm{Re}}=\frac{\tau_{p} \eta}{\rho h^{2}},
$$

where $h$ is the shortest dimension setting the shear rate, expresses the relative importance of elastic to inertial effects. Significantly, El depends only on geometry and material properties of the fluid, and is independent of flow rate. The small dimensions $h$ inherent in microfluidic devices allow high-El flows to be established more easily; comparably elastic flows in macroscopic experiments would require significantly more viscous solvents. As a result, microfluidic devices have opened up heretofore unexplored regions in Re-De space for viscoelastic flows. For example, Rodd et al. (2004) explore new regimes in viscoelastic contraction flows, shown in Fig. 25.
[For a review of contraction flows of viscoelastic fluids, see Boger (1987).]

The coil-stretch transition. As shear rates increase and Wi becomes $\mathcal{O}(1)$, fluid forces overcome the restoring forces of the entropic spring, causing the polymer to unravel to a stretched state (de Gennes, 1974; Hinch, 1977; Bird, Hassager, et al., 1987; Larson and Magda, 1989; Schroeder et al., 2003). In fact, the model Hookean spring used here extends indefinitely, which is clearly unphysical. Various remedies are employed in flow simulations, such as the finitely extensible, nonlinearly elastic (FENE) dumbbell (Bird, Hassager, et al., 1987; Larson, 1988, 1999; Rallison and Hinch, 1988). Nonlinear elastic effects follow from many polymer models; singlemolecule experiments indicate that the wormlike chain captures DNA elasticity particularly well (Smith et al., 1992; Bustamante et al., 1994; Vologodskii, 1994; Marko and Siggia, 1995; Meiners and Quake, 2000).

Normal stress differences. Polymers introduce an anisotropic component to the normal stress: an extended polymer exerts a tension along its axis of extension, so that the stress on a surface perpendicular to the extension is lower than that on the surfaces parallel. Shear flows are similar but more complicated, since there exist three different normal stresses: in the flow direction, in the gradient direction, and in the vorticity direction (perpendicular to both), giving two normal stress differences. These normal stress differences are responsible for many of the remarkable properties of non-Newtonian fluids (Bird, Armstrong, and Hassager, 1987).

Flow modifications. Neglecting Brownian motion, each bead is advected with the flow and driven by the restoring spring force. Advection involves moving with the local fluid environment, and thus exerts no force on the fluid. By contrast, the spring must exert a force to pull the bead through its environment. Thus in the far field, each deformed polymer contributes a force-dipole stresslet flow to the background. These stresslets form the basis for a flow computational technique (Phillips, 2003). In the dilute, weakly elastic limit, this perturbation is small. As elastic effects become stronger, however, these perturbations become significant as well, and the coupling between polymeric stress and fluid flow becomes strongly nonlinear.

Flow memory. Flows strain suspended polymers, which exert an elastic stress back on the fluid. The finite polymer relaxation time $\tau_{p}$ gives memory to the fluid, as the conformation of each polymer depends on the history of the flow. This stands in contrast with Newtonian Stokes flows, which are uniquely determined at any instant by boundary conditions and external forcing.

Constitutive equation for macroscale flows. The suspension of Hookean dumbbells in a viscous solvent is one of very few models for polymeric microstructure that yields a closed-form macroscopic constitutive equation, the Oldroyd-B equation (Oldroyd, 1950; Bird, Hassager, et al., 1987; Larson, 1999). This equation accounts for the coupled evolution of the fluid flow and the polymeric stress tensor, and has been called the hydrogen atom of elastic fluids. Although shortcomings certainly 
exist such as the unbounded high-Wi extension described above, the Oldroyd-B equation provides the simplest description of elastic fluids and captures many features of elastic flow behaviors qualitatively and semiquantitatively. However, note that when device dimensions get very small on the order of the length of stretched polymers, such continuum models are not likely to be appropriate to describe such flows.

\section{Microfluidic single-polymer studies}

The low Reynolds numbers inherent in microfluidic devices allow one to study the dynamics of individual polymer molecules in precisely defined flows. This permits direct tests of models for polymer dynamics, and complements parallel studies using Brownian dynamics. Furthermore, single-molecule studies (experimental and theoretical) reveal significant fluctuations that would be difficult or impossible to measure from bulk studies. A central goal of research into complex fluids involves relating macroscale behavior to microscale structure, and microfluidic devices show considerable potential towards this end.

A variety of single-molecule polymer studies have been performed in microfluidic devices. Early singlepolymer studies did not involve a flow so much as a polymer held fixed in a moving bath. Using these techniques, the elasticity (Smith et al., 1992) and relaxation times of individual DNA molecules were measured (Perkins et al., 1994, 1995), with complementary simulations (Quake, 1994; Larson et al., 1997). These experiments demonstrated that internal hydrodynamic interactions remain significant even at large extensions. Experiments on single DNA molecules in shear flows revealed an average polymer shape that was approximately ellipsoidal, as expected. Individual polymers, however, showed molecular individualism (de Gennes, 1997): large, aperiodic extension and tumbling motions, seen both in experiments (Smith et al., 1999; Doyle et al., 2000; Ladoux and Doyle, 2000) and in Brownian dynamics simulations (Liu, 1989; Hur et al., 2000). Likewise, the relation between microscopic and macroscopic polymeric fluid behavior was investigated in a microfluidic shear cell by fluorescently labeling a small number of probe polymers in a solution of identical, invisible polymers (Babcock et al., 2000). Individual DNA molecules in Poiseuille flow in a small capillary (Manneville et al., 1996) unravel in a manner consistent with the stem and flower theory for polymer extension (Brochard-Wyart, 1995). Microfluidic flows have been employed to stretch DNA and tether it at both ends, allowing the transverse fluctuations to be studied (Crut et al., 2003; Gueroui et al., 2003).

Polymer dynamics in extensional flows were studied using crossed microchannels, as shown in Fig. 26 (Perkins et al., 1997; Smith and Chu, 1998; Schroeder et al., 2003) or flow focusing (Wong et al., 2003), and were complemented by Brownian dynamics studies (Larson et al., 1999; Maroja et al., 2001; Hur et al., 2002). These studies revealed a more subtle coil-stretch transition than originally envisioned, due in part to the multiple

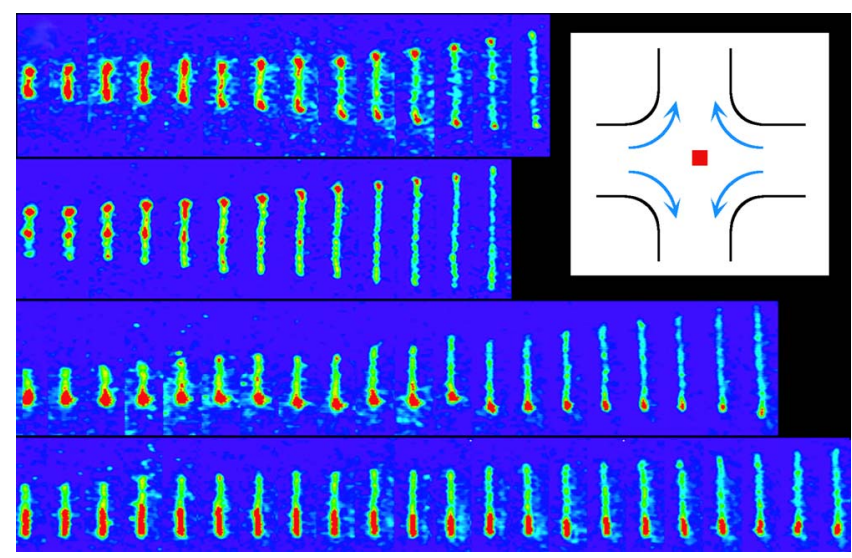

FIG. 26. (Color in online edition) Single fluorescently labeled DNA molecules subjected to a microfluidic elongational flow allow a direct visualization of the molecular individualism of polymers, which unravel in a number of characteristic modes. Four such modes are shown evolving left to right. Inset: extensional flow established at a microchannel cross junction. Reprinted with permission from Perkins et al., 1997. (C)1997, AAAS.

energetically similar conformations shown in Fig. 26 (de Gennes, 1997; Perkins et al., 1997). The coil-stretch transition was predicted to be hysteretic because the friction $\xi$ increases as the polymer unravels and thus $\tau_{p}$ increases; thus a lower extension rate $\dot{e}$ is required to maintain a stretched polymer (de Gennes, 1974; Hinch, 1977). On the other hand, the strong increase of $k_{H}$ with polymer extension shortens the relaxation time, which could mask or overwhelm any hysteresis in most practical situations (Meiners and Quake, 2000). However, hysteresis was observed in single-molecule experiments in shallow channels (Schroeder et al., 2003).

\section{Elastic instabilities}

Not surprisingly, non-Newtonian fluids display a host of flow instabilities, even in the absence of inertia (Pearson, 1976; Petrie and Denn, 1976; Larson, 1992). Historically, quantitative understanding has been difficult, in part because typical non-Newtonian fluids have a shearrate dependent viscosity (most frequently shear thinning). That shear thinning would exacerbate instabilities makes sense: locally increasing the shear rate lowers the local viscosity, which makes the fluid easier to shear, and so on. It is thus frequently difficult to tease apart the effects of shear thinning and elasticity. However, the discovery of highly elastic, but constant-viscosity, Boger fluids (Boger, 1977; Boger and Yeow, 1992) has facilitated the study of purely elastic effects.

Instabilities have been discovered that are purely elastic in nature, occurring at a critical De or We, even as $\mathrm{Re} \rightarrow 0$ (McKinley et al., 1996; Shaqfeh, 1996). Such elastic instabilities were originally identified in TaylorCouette flow (Muller et al., 1989; Larson et al., 1990, 1994; Shaqfeh et al., 1992; Baumert and Muller, 1995; Graham, 1998), and occur additionally in Dean flow (Joo and Shaqfeh, 1991, 1992), lid-driven cavity flow (Pakdel 
and McKinley, 1996), cone-plate and coaxial disk flows (Phan-Thien, 1983, 1985; McKinley et al., 1991, 1995; Olagunju, 1995), rotating parallel plates (McKinley et al., 1991; Byars et al., 1994), in the wake behind a confined circular cylinders (McKinley et al., 1993), and in free surfaces that buckle (Kumar and Graham, 2000; Graham, 2003). The influence of viscous heating on this instability introduces an additional dimensionless parameter (the Nahme number), whose influence has been examined theoretically and experimentally (Al-Mubaiyedh et al., 1999; White and Muller, 2000, 2003; Rothstein and McKinley, 2001).

Curved streamlines are an almost ubiquitous ingredient of elastic instabilities. Generically, polymers that have been extended along curved streamlines are taken by fluctuations across either shear-rate gradients or tension gradients in the base state. This couples the hoop stress $\sigma_{\theta \theta}$ acting along the curved streamline to the radial and axial flows, which can amplify the perturbation. Such elastic instabilities in a saturated nonlinear state give rise to irregular fluctuations and elastic turbulence (Groisman and Steinberg, 2000, 2004; Larson, 2000), which has been exploited to enhance microfluidic mixing (Groisman and Steinberg, 2001).

Only recently have such elastic nonlinearities been exploited in microfluidic flows. Efforts in decades past to design and construct nonlinear fluidic logic elements resulted in such common items as pulsating shower heads, windshield wipers, and sprinkler systems (Humphrey and Tarumoto, 1965; Foster and Parker, 1970; Joyce, 1983). Attempts to build miniaturized fluidic computers, however, foundered, since inertial nonlinearities vanish as devices were scaled down and $\mathrm{Re} \rightarrow 0$. Elastic instabilities occur at vanishingly small Reynolds number, and can reintroduce flow nonlinearities in microfluidic devices. With microfluidic computing in mind, several nonlinear microfluidic flow elements have been designed that exploit elastic nonlinearities: a nonlinear fluid resistor (Fig. 27), a bistable flip-flop memory element (Fig. 28), and a flow rectifier (Fig. 29; Groisman et al., 2003; Groisman and Quake, 2004).

We consider first the nonlinear flow resistor (Fig. 27). Newtonian low-Re flows vary linearly with pressure, regardless of channel geometry. By contrast, dilute polymer solutions behave linearly only for small applied pressures, for which $\mathrm{Wi} \ll 1$ and polymers deform only slightly. Above a threshold pressure, however, the nonlinear flow resistor passes a nearly constant flow of elastic fluid over nearly a decade increase in applied pressure. This constant flow regime occurs once $\mathrm{Wi}>1$, which can be understood qualitatively from energy arguments. Under such strong flows, additional power input that would normally increase the flow rate is instead consumed by further deformation of the polymers (Groisman et al., 2003). This deformation is stored entropically but is not returned to the flow due to the hysteretic differences between coil stretching and relaxation.

The fluidic flip-flop memory element is bistable, with oppositely directed fluids into a four-channel junction (a)

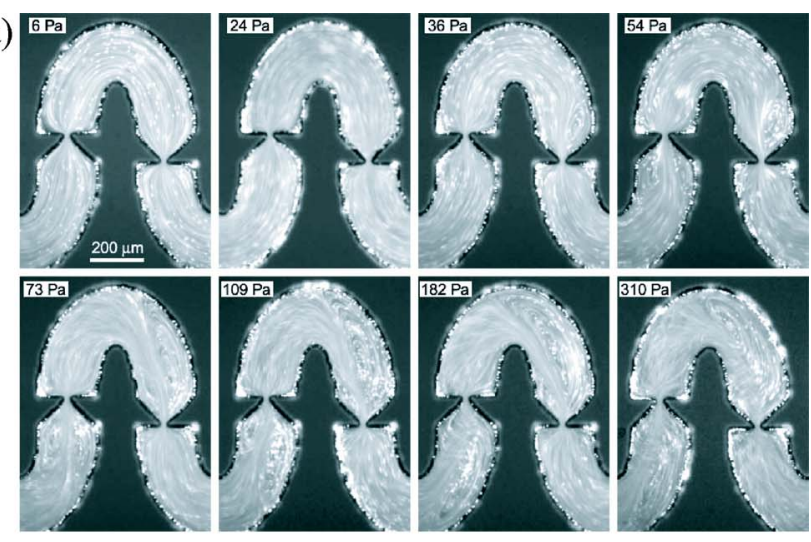

(b)

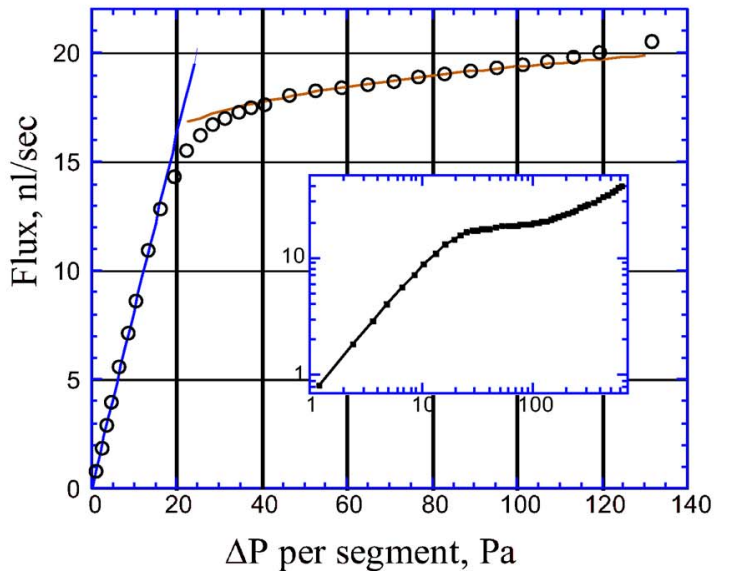

FIG. 27. (Color in online edition) Nonlinear viscoelastic flow resistor. While a low-Re Newtonian fluid would exhibit a linear pressure-flow relationship, elastic stresses in dilute polymer solutions introduce significant nonlinearities, even at very small Re. (a) Elastic nonlinearities cause an increasingly complex flow as pressure increases. A vortex forms on the upstream side of the contraction between applied pressures 24 and 36 $\mathrm{Pa}$, which corresponds to the elbow in the flow rate vs pressure plot (b). The contraction vortex extends upstream and increases in size as pressure increases. (b) The flux vs pressure profile is linear for low applied pressures, but flattens for applied pressures above a critical value (where $\mathrm{Wi}>1$ ), creating an approximately constant current source. Reprinted with permission from Groisman et al., 2003. (02003, AAAS.
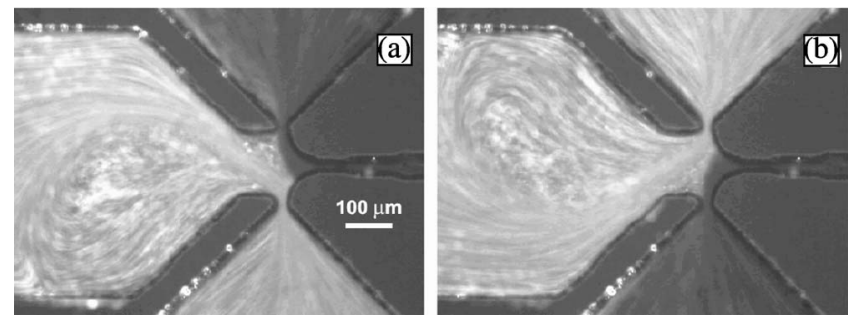

FIG. 28. Nonlinear viscoelastic flip-flop memory device. Dilute polymer solutions (one dark, one light) that enter a junction from opposite sides choose an exit channel, contrasting with Newtonian fluids that split to flow through both. The fluids flow stably through the exit they have chosen, but switch when a transient $(50 \mathrm{~ms})$ pressure pulse is applied. Reprinted with permission from Groisman et al., 2003. (2003, AAAS. 

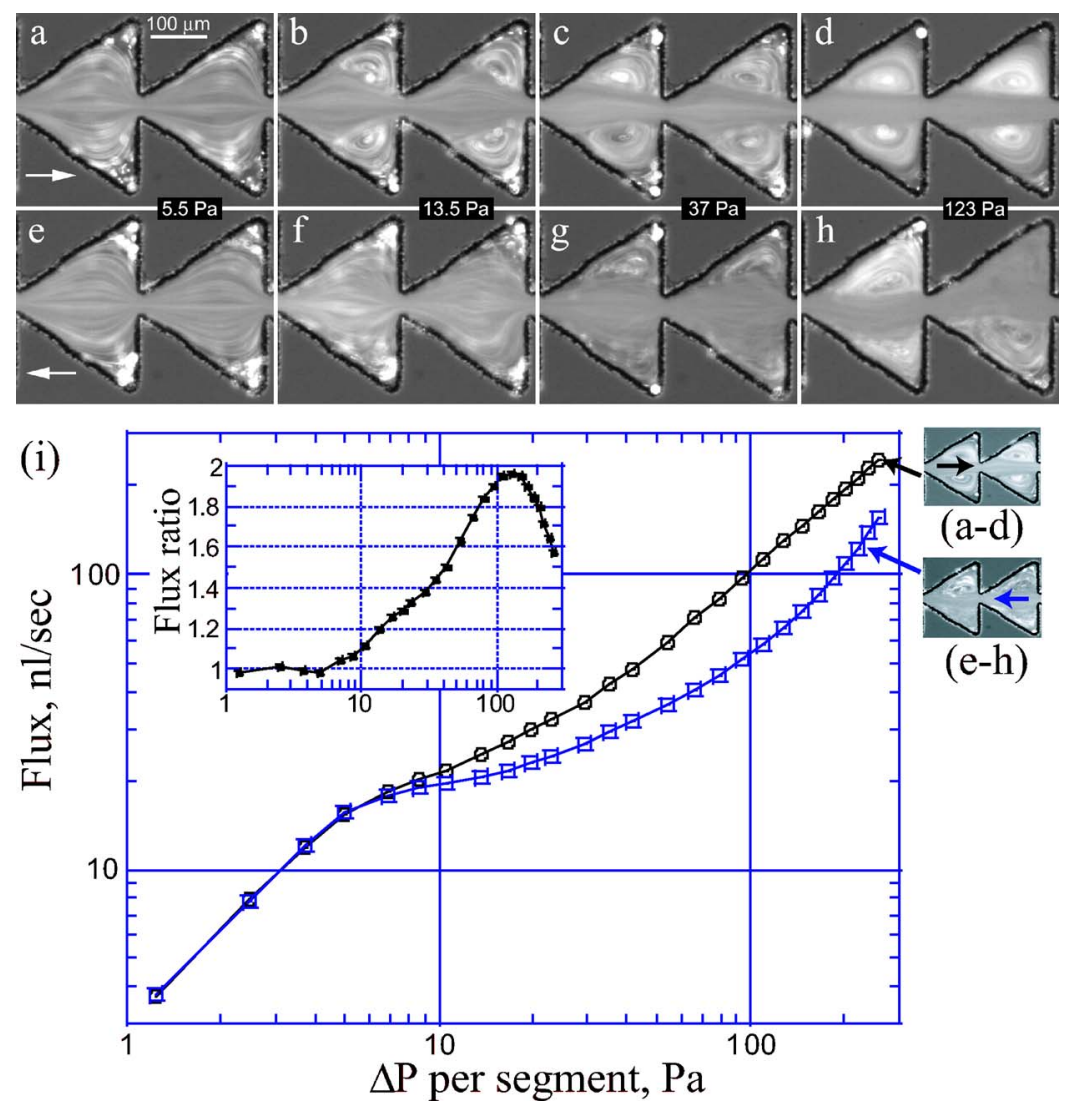

FIG. 29. (Color in online edition) Viscoelastic fluidic rectifier. Low-Re Newtonian fluid flows are reversible and indifferent to the flow direction. By contrast, elastic stresses in dilute polymeric solutions partially rectify such flows, leading to quantitative and qualitative differences. (a)-(d) show dilute polymer solution flows in the forward direction, and (e)-(h) in the reverse direction. (i) Above a critical pressure, flow rates in the forward direction begin to exceed those in reverse, giving a flux ratio (inset) that can be as high as 2. Reprinted with permission from Groisman and Quake, 2004.

(Fig. 28). Macroscopic flows through a related crossed geometry were studied by Cochrane et al. (1981), albeit without mention of bistability or applications. If the fluids were Newtonian, each stream would simply split and flow evenly into both outlet channels. Non-Newtonian fluid streams, on the other hand, break symmetry and choose one of the two. The junction remembers this state due to the stability of the flow, but can be switched using a transient pressure shock (Groisman et al., 2003). This effect is likely related to the "tubeless siphon" phenomenon exhibited by polymeric fluids (Bird, Armstrong, and Hassager, 1987), in which polymers stretched and aligned with the flow exert an elastic stress that tends to pull neighboring molecules in the flow direction.

Last, we examine the flow rectifier shown in Fig. 29. Linear, low-Re Newtonian flows behave identically when forced forward or backward through a channel. Polymeric flows, on the other hand, can be rectified by elastic stresses, whereupon an applied pressure $\Delta p$ drives a different flow rate in one direction than the other. The channel asymmetry causes advecting polymers to experience a different flow history for forward and backward flows, which changes the molecular response and macroscopic stress. This leads to a partial rectification in the device whereby the pressure differ- ences needed to drive a given flow rate forward and backward differ by as much as a factor of 2 .

\section{Controlled polymer deformation for separations}

Separating biopolymers like DNA and proteins is of utmost importance for many applications and disciplines, including biology, biochemistry, forensic science, drug design, genome sequencing, proteomics, and so on. As such, many techniques have been developed to separate polymers by size, of which various electrophoretic techniques are standard. While we discuss electrokinetic effects in more detail in Sec. III.A, we note here an important and rather surprising property of electrophoresis: the electrophoretic mobility $\mu_{E}$ of an object (relating its velocity to applied field strength, $\mathbf{u}=\mu_{E} \mathbf{E}$ ) is independent of its size. Among many counterintuitive consequences is that electrophoresis in aqueous solution can separate objects by surface charge but not size.

As a result, electrophoretic polymer separations are typically performed in gels, wherein the resistance to polymer motion increases for longer polymers. Viovy (2000) and Slater et al. $(2002,2003)$ provide excellent reviews of the physics behind polymer electrophoresis, Ugaz et al. (2004) provides a nice overview of various techniques in a microfluidic context, and Tegenfeldt et 

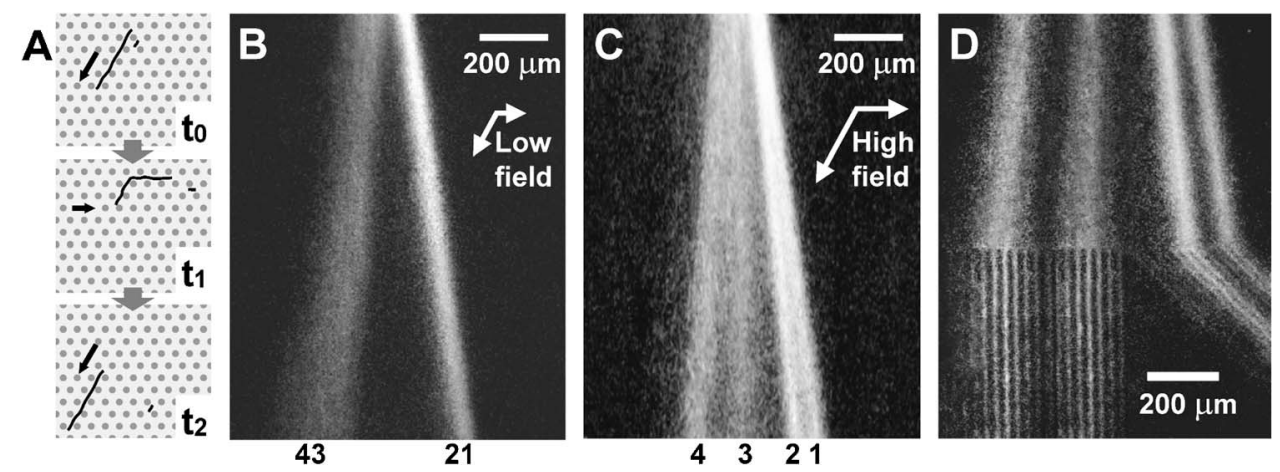

FIG. 30. DNA molecules of different lengths naturally follow different trajectories through a pulsed-field electrophoretic DNA prism. (A) An applied electric field extends polymer molecules in the direction of the field at time $t_{0}$. When an electric field is applied at an oblique angle $\left(t_{1}\right)$, shorter polymers encounter fewer obstacles and travel more easily along the second field direction than longer polymers. (B)-(D) Separation of (1) $61 \mathrm{~kb}$, (2) $114 \mathrm{~kb}$, (3) $158 \mathrm{~kb}$, (4) $209 \mathrm{~kb}$ DNA with fields of varying strength. (B) 250-ms pulses with field strengths 32 and $20 \mathrm{~V} / \mathrm{cm}$ are strong enough to separate the DNA into two bands. (C) Stronger fields (240 and $150 \mathrm{~V} / \mathrm{cm}, 40-\mathrm{ms}$ pulses) separate the DNA into four distinct bands. (D) Microfludic channels at the outlets collect the separated DNA. Reprinted with permission from Huang, Tegenfeldt, et al., 2002. C2002, NPG.

al. (2004) describes separation efforts using microfabricated matrices. We do not attempt to cover the tremendous effort towards microfluidic separations, but rather refer the reader to existing reviews focusing on such devices (Bousse et al., 2000; Bruin, 2000; Auroux et al., 2002), on electrophoretic separation techniques for DNA (Dolnik et al., 2000; Viovy, 2000; Slater et al., 2002; Ashton et al., 2003; Barbier and Viovy, 2003; Landers, 2003; Sartori et al., 2003), and for proteins (Dolnik, 1997, 1999; Dolnik and Hutterer, 2001; Figeys and Pinto, 2001; Hancock et al., 2002; Lion et al., 2003).

Different electrophoretic strategies utilize differing degrees of polymer deformation and relaxation, and can thus be connected to Wi and De. Polymers with size $R_{0}$ that are small relative to the gel's pore size $b_{g}$ do not deform significantly and flow freely without separating, whereas collisions and entanglement between the polymer and gel depend strongly on polymer length when $R_{0} \sim b_{g}$, causing strong separation. However, when $R_{0}$ $\gg b_{g}$, the polymer must reptate in a highly extended state to move, and once again its mobility is insensitive to length. Periodically changing the field direction (pulsed-field electrophoresis) exploits the lengthdependent drag when such highly extended polymers are driven perpendicular to their extension.

Through microfabrication, a well-characterized set of obstacles can be created to allow a more systematic study of the physics of polymer electrophoresis, with the aim of rational and systematic improvement of separation systems (Volkmuth and Austin, 1992; Austin and Volkmuth, 1993; Chou et al., 2000; Tegenfeldt et al., 2004). Systems have been developed to exploit controlled polymer deformation about microfabricated posts (Volkmuth and Austin, 1992; Austin and Volkmuth, 1993; Volkmuth et al., 1994; Duke et al., 1996, 1997; Chou et al., 2000; Bakajin et al., 2001; Huang, Tegenfeldt, et al., 2002) or self-assembling posts of magnetic beads (Doyle et al., 2002). For example, Fig. 30 depicts a microfabricated array used as a pulsed-field electrophoretic DNA prism (Huang, Tegenfeldt, et al., 2002). Because smaller fragments travel further along the secondary field direction than longer ones, the angle of travel of a polymer depends on its length (analogous to light in a prism), allowing fragments to be sorted by length into an array of microchannels. Theoretical efforts include the Taylor dispersion of DNA in such systems (Dorfman and Viovy, 2004) and Brownian dynamics simulations. The latter suggest that randomly placed posts are better suited for polymer separations than regular arrays, in the dilute limit (Patel and Shaqfeh, 2003). These and other techniques [such as entropic trapping and separation (Turner et al., 2002)] are reviewed by Slater et al. (2002).

Finally, we note that these microfabricated array studies complement single-molecule studies of DNA under flow, because holding a polymer in place against electrophoretic driving mimics holding a polymer in place against a background flow (Long et al., 1996; Bakajin et al., 1998). Furthermore, thin "slit" channels allow the effect of molecular confinement on polymers to be studied, both experimentally, and theoretically (Brochard and de Gennes, 1977; Bakajin et al., 1998; Dimalanta et al., 2003; Jendrejack, Jendrejack, Schwartz, et al., 2003; Jendrejack et al., 2004; Woo et al., 2004a, 2004b).

\section{E. The Grashof and Rayleigh numbers: Tracer-driven flows}

Thus far, gravity has played no role in our discussion. If fluids of different densities are brought together, however, gravity may play a role. In order to understand, design, and utilize such systems, one must understand the effects that density differences have on fluid behavior. To explore these effects, consider the channel shown in Fig. 31. The fluid in one half of the channel contains dense solute molecules at a concentration $c$, whereas the fluid in the other half is clear. We assume solution density differences vary linearly with solute concentration differences via 


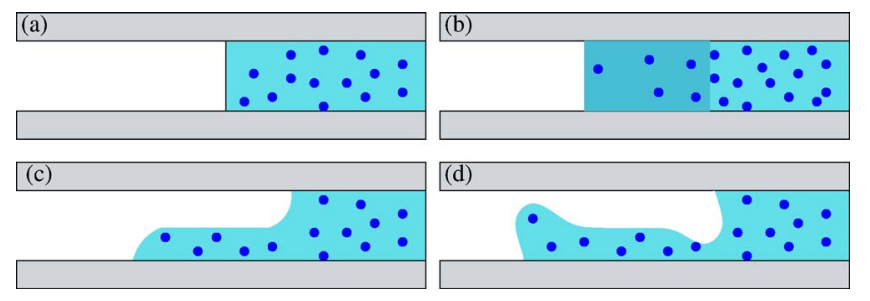

FIG. 31. (Color in online edition) Free-interface diffusion in protein crystallization. (a) Initial configuration. The interface evolution for varying regimes of buoyant flow: (b) $\mathrm{Ra} \ll 1, \mathrm{Gr}$ $\ll 1$, where diffusion dominates and the interface spreads diffusively; (c) $\mathrm{Ra} \gg 1, \mathrm{Gr} \ll 1$, where advective transport dominates and tongues of each fluid are driven into the other; and (d) $\mathrm{Ra} \gg 1, \mathrm{Gr} \gg 1$, where inertia drives the gravity currents to splash.

$$
\Delta \rho=\rho_{0} \alpha_{c} \Delta c
$$

where $\rho_{0}$ is the density of the clear liquid. An analogous system involving thermal (rather than solute) gradients is obtained when a temperature field $\Delta T$ replaces the solute concentration field $\Delta c$, in which case $\alpha_{c}=\alpha_{T}$ is the coefficient of thermal expansion.

The system in Fig. 31 is clearly unstable, as buoyant forces drive the dense fluid downhill into the less dense fluid, and vice versa. The system could evolve in several ways, depending on channel height $h$ and fluid properties. At issue is the fact that buoyancy-driven fluid velocities are not known at the outset, but are instead determined by the distribution of buoyant forces acting on the fluid. To obtain a scaling estimate, we assume a quasisteady force balance, where viscous forces $\left(f_{v}\right.$ $\left.\sim \eta U_{b} / h^{2}\right)$ balance buoyant forces $\left(f_{b} \sim \alpha_{c} \rho_{0} \Delta c g\right)$ to give a buoyant velocity scale

$$
U_{b} \sim \frac{\alpha_{c} \rho_{0} \Delta c g h^{2}}{\eta} .
$$

With the buoyant velocity scale $U_{b}$, we can determine whether convection or diffusion dominates the solute transport. The ratio of convective to diffusive flux is given by

$$
\frac{\text { convective flux }}{\text { diffusive flux }}=\frac{U_{b} h}{D} \sim \frac{\alpha_{c} \rho_{0} \Delta c g h^{3}}{\eta D} \equiv \mathrm{Ra},
$$

and is termed the Rayleigh number $\mathrm{Ra}$. When $\mathrm{Ra} \ll 1$, diffusion dominates over advection and the interface spreads diffusively, as shown in Fig. 31(b). If, on the other hand, the flow is fast and outcompetes diffusion $(\mathrm{Ra} \gg 1)$, then a gravity current of each fluid penetrates the other, as shown in Fig. 31(c). Figure 31(d) shows an even faster flow, where inertia causes the fingers to splash. For this to occur, inertial forces must be significant relative to viscous forces, which requires the Grashof number Gr, defined by

$$
\frac{f_{i}}{f_{v}} \sim \frac{\rho_{0} U_{b} h}{\eta} \sim \frac{\Delta c \alpha_{c} \rho_{0}^{2} g h^{3}}{\eta^{2}} \equiv \mathrm{Gr},
$$

to be large.
One might complain that the Rayleigh and Grashof numbers are little more than Reynolds and Péclet numbers in slight disguise. The criticism is valid, since the two pairs of dimensionless numbers compare the same fundamental effects. The distinction lies in the fact that in buoyancy-driven flows, the tracers are themselves the agents responsible for driving the flow. Systems containing truly passive tracers whose behavior is determined by $\mathrm{Pe}$, differ significantly from those containing buoyant or dense tracers. In other words, a high Pe flow looks very different than a high Ra flow.

In fact, a similarly artificial distinction was made with less fanfare between the Péclet number and the Reynolds number itself. After all, the Reynolds number can be viewed as a Péclet number for vorticity: at low Re, Stokes flow solves the vorticity diffusion equation, whereas advection of vorticity becomes more significant as Re increases. With Re, just as with $\mathrm{Ra}$ and $\mathrm{Gr}$, the distinction with $\mathrm{Pe}$ is relevant precisely because the tracer, whether vorticity or density, acts back on the flow.

For this reason, these numbers are related,

$$
\frac{\mathrm{Pe}}{\mathrm{Re}} \equiv \frac{\mathrm{Ra}}{\mathrm{Gr}}=\frac{\nu}{D},
$$

and their ratios reflect the ratios of diffusivities for the relevant tracers. The ratio of vorticity diffusivity $\nu$ to solute diffusivity $D$ is called the Schmidt number Sc $=\eta / D$, whereas the ratio of $\nu$ to thermal diffusivity $\kappa_{T}$ is the Prandtl number $\operatorname{Pr}=\nu / \kappa_{T}$.

In what follows, we explore a few examples where microfluidics exploits or allows one to neglect the effects of buoyancy.

\section{Protein crystallization}

Protein crystallization is an application of tremendous importance to structural biology and biotechnology [see, e.g., McPherson (1999)], and one in which density gradients in solution can play a significant role. Although DNA sequencing reveals the amino acid sequence of proteins, little to nothing can be said about the protein's actual function unless its folded structure is known. Unfortunately, despite decades of research, predicting the structure of the protein from its sequence remains notoriously difficult, even for the smallest of proteins. At present, the most popular method for resolving such structures involves x-ray crystallography, which requires large, high-quality protein crystals to be grown. This, too, has proven difficult, in part because the parameter space is so vast (temperature, protein concentration, precipitant identity and concentration, $p \mathrm{H}$, etc.). Protein crystallization is typically attempted using an intuitively guided but massive sweep through parameter spaceessentially trial and error. Such extensive parameter sweeps consume large quantities of time, effort, and material-which may be expensive, difficult, or even impossible to acquire.

Microfluidics is ideally suited for such a task, as it allows automated, parallel sweeps through parameter space using far less material than conventional 


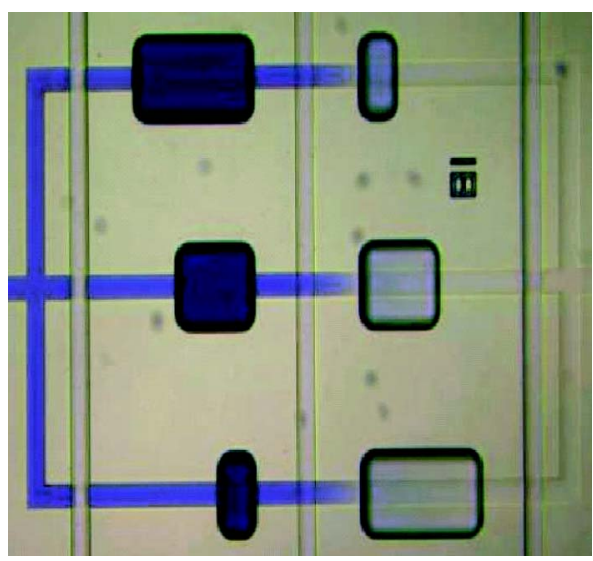

FIG. 32. (Color in online edition) Low $\mathrm{Gr}$ proteincrystallization chip (Hansen et al., 2002). Protein and buffer solutions fill opposing sets of chambers, which are initially isolated by a central valve. Here the valve is opened, and the interface evolves diffusively due to the low $\mathrm{Gr}$ and $\mathrm{Ra}$ in the system. The slow evolution of the resulting solutions (spanning a range in parameter space) allows protein crystals to nucleate and grow. Figure courtesy of C. Hansen.

(macro)techniques (Hansen and Quake, 2003; Zheng et al., 2003). For example, the microfluidic device of Hansen et al. (2004) rapidly and systematically explores the phase space for protein solubility with minimal reagent consumption. Furthermore, the small dimensions of microfluidic devices allows for different fluid physics than are found in macroscopic treatments. This can play an important role in crystallization techniques such as free-interface diffusion (Salemme, 1972; Ng et al., 2003) and can be used to manipulate the kinetics of nucleation and growth.

Figure 32 shows a microfluidic device designed to crystallize proteins by free-interface diffusion (Hansen $e t$ al., 2002). Two sets of chambers of varying volume are separated by long, narrow channels separated by valves. One set of chambers is filled with a dense protein solution of known concentration, and the other is filled by a solution with a concentration of crystallizing agents. The valves connecting each pair of chambers have been opened, and the interface between the two solutions evolves, as in Fig. 31. Gradually, the contents of each chamber make their way into the other, and a swath of trajectories through $\left(c_{p}, c_{s}\right)$ parameter space is explored. Free-interface diffusion therefore allows many conditions to be explored in a single experiment, and also traces out a path for crystals to nucleate and grow. This is achieved by ensuring that the interface spreads diffusively [Fig. 31(b)], which requires both Ra and Gr to be small. The small dimensions of microfluidic devices allow the $\mathrm{Ra} \ll 1, \mathrm{Gr} \ll 1$ regime to be easily reached. Previously, this was achieved by taking samples into space to decrease $g$ (DeLucas et al., 1989, 2002; McPherson et al., 1999; Lee and Chernov, 2002), which does not allow for the highly parallel studies required for de novo crystallization, or using an agarose gel to suppress convection (Fig. 33; Garcia-Ruiz et al., 2001; Ng et al., 2003). (a)
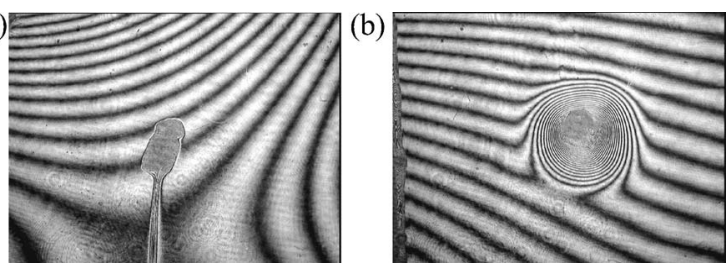

FIG. 33. Interferometric images of crystal melting. (a) Dense fluid is formed in the vicinity of a dissolving crystal and falls in a wake, indicating high Gr. Crystal growth suffers the opposite problem: buoyant fluid forms around the crystal as dense molecules are locally removed from solution, establishing a convective flow that adversely affects crystal quality. (b) Dissolution occurs more isotropically when the crystal is held in a $0.1 \%$ (weight/volume) agarose gel, which suppresses convection. Reprinted with permission from Garcia-Ruiz et al., 2001. (C)2001, Elsevier.

While all of these methods have been useful in understanding the basic physics of protein crystallization, as of this writing, only the microfluidic format has been successfully adapted to high-throughput screening.

An additional factor complicating the growth of protein crystals is that the molecules themselves tend to be larger, more complicated, and softer than atoms found in solid-state crystals familiar to most physicists. It is therefore comparatively easier to disrupt protein crystallization with defects, impurities, or even slight mismatches in molecular orientation. One factor that is believed to adversely affect protein crystal quality is fluid convection. Since solute protein is depleted near the crystal, solution near the crystal can drive a buoyant flow if $\mathrm{Gr}$ and $\mathrm{Ra}$ are not low enough, which introduces a number of effects involving impurities and inhomogeneous growth (Grant and Saville, 1991; Vekilov et al., 1998; Lee and Chernov, 2002). (The inverse effect, wherein the dense layer of fluid that forms around a dissolving crystal falls in a wake, is shown in Fig. 33.) For this and the other reasons enumerated above, microfluidic devices are expected to have a decisive advantage and to become the standard in large-scale structural biology efforts.

While spatially inhomogeneous growth due to buoyant flow is undesirable for protein crystals, an analogous mechanism has been exploited to easily fabricate sharp optical probe tips, shown in Fig. 34 (Lambelet et al., 1998; Unger et al., 1998; Stockle et al., 1999). An etchant solution eats away at an optical fiber confined to a tube. The dense product-laden/etchant-sparse fluid falls down along the tube center, and product-free/etchant-rich fluid moves upwards towards the fiber edges. The flow drives a preferential etching of the fiber edges, providing positive feedback that further reinforces the flow. This technique requires large $\mathrm{Ra}$ (due to the concentration of dense product) to drive the flow, but small enough $\mathrm{Gr}$ for the flow to be regular. The etchant is advected with, and diffuses through, the buoyancy-driven flow set up by the etch products. Thus Pe for the etchant must be large; otherwise, diffusion would homogenize the etchant concentration, decoupling the etch process from the flow, 

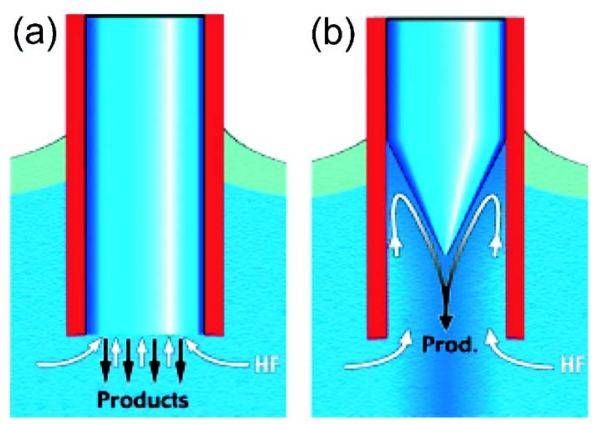

FIG. 34. (Color in online edition) A phenomenon similar to that in Fig. 33(a) is exploited in tube etching, a technique for fabricating sharp optical fiber tips. (a) The dense products of an etching reaction fall along the tube axis. (b) This drives a fluid flow that delivers "fresh" etchant to the fiber edges, concentrates the etched products towards the center, and reinforces the fluid flow. Reprinted with permission from Stockle et al., 1999. (C)1999, AIP.

and no sharp tip would be formed. Finally, we note that phenomena similar to Figs. 33 and 34 are observed in electrodeposition (Huth et al., 1995). The analogy between electrohydrodynamics and buoyancy-driven flows will be pursued shortly.

\section{Polymerase chain reaction (PCR)}

Polymerse chain reaction (PCR) is a process used to exponentially replicate double-stranded DNA, allowing even a very small amount of DNA to be amplified into a sufficient amount for sophisticated analysis. PCR involves a three-step thermal cycle in the presence of a reagent soup: (i) heating the solution to melt the DNA by separating each ds-DNA into two single strands; (ii) cooling so that DNA primers and DNA polymerase enzymes bind each strand (annealing); and (iii) warming slightly to promote the base-by-base DNA replication by the polymerase (extension). Ideally, each PCR cycle doubles the number of double-stranded DNA molecules.

Integrating PCR into microfluidic devices has been achieved by many groups, typically by cycling the temperature of a microfluidic sample to replicate the standard macroscopic PCR (Wilding et al., 1994; Burns et al., 1996; Cheng et al., 1996; Woolley et al., 1996; Schmalzing et al., 1997; Belgrader et al., 1999; Khandurina et al., 2000; Chiou et al., 2001; Hong et al., 2001; Lagally, Emrich, and Mathies, 2001; Lagally, Medintz, and Mathies, 2001; Auroux et al., 2004). An alternate strategy involves pumping solution through various temperature zones to mimic PCR [Fig. 35(a)] (Kopp et al., 1998; Liu, Enzelberger, and Quake, 2002), whose benefit is that cycle time no longer depends on the time required to heat or cool the solution and its surroundings. Another approach exploits high-Ra buoyant flows to perform PCR in a steady temperature profile without an external pump (Krishnan et al., 2002; Braun et al., 2003). The basic idea involves establishing a large convective flow whose roll fills the experimental cell. This flow advects

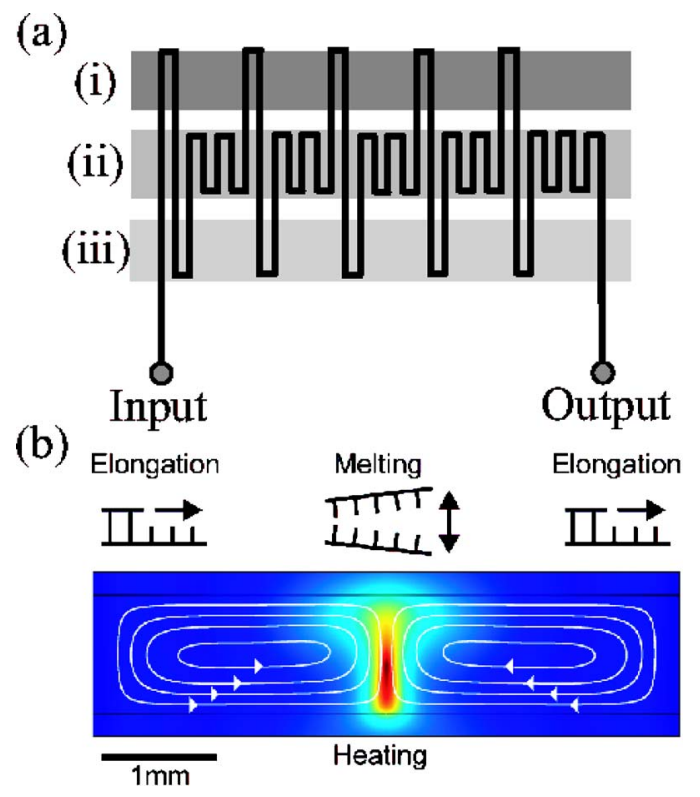

FIG. 35. (Color in online edition) Polymerase chain reaction (PCR) in a steady temperature field. (a) Solution is driven along a channel that winds through temperature regions (i), (ii), and (iii) designed to cause DNA melting, extension, and annealing. Thus the temperature profile seen by the solution matches a standard PCR cycle. Reprinted with permission from Kopp et al., 1998. (C1998 AAAS. (b) A PCR reactor in which temperature gradients drive a convective fluid flow that takes suspended DNA molecules through a temperature profile designed to resemble that of PCR. Reprinted with permission from Braun et al., 2003.

DNA molecules $(\mathrm{Pe} \gg 1)$ through the variable temperature profile in the fluid. By properly designing the experiment, the temperature profile experienced by DNA can be made to resemble that of conventional PCR, thus allowing the chain reaction to proceed. The underlying convective flows have been established in two ways: (i) uniformly heating the bottom plate and cooling the top plate enough to drive Rayleigh-Benard convection at $\mathrm{Ra} \sim 10^{6}$ (Krishnan et al., 2002), or (ii) heating the fluid inhomogeneously [Fig. 35(b)] at $\mathrm{Ra} \sim 10^{4}$ to establish laminar convective flow (Braun et al., 2003). Finally, DNA molecules advect with the flow along the bottom of the cell towards the center of the roll, but also move outwards via thermophoresis (itself poorly understood), leading to trapping in a ring so long as $\mathrm{Pe} \gg 1$ (Braun and Libchaber, 2002).

\section{Electrohydrodynamic instabilities}

We have presented two cases where $\mathrm{Ra}$ and $\mathrm{Gr}$ are relevant for describing the resulting flow. Solution density gradients (however they arise) couple to an external field (gravity) to drive a fluid flow that in turn advects the density gradients. We now discuss an interesting variant on this picture, involving electrical Rayleigh and Grashof numbers. Conductivity gradients in solution couple to electric fields to drive a fluid flow that, in turn, advects those same gradients. Physically, the (local) con- 
(a)

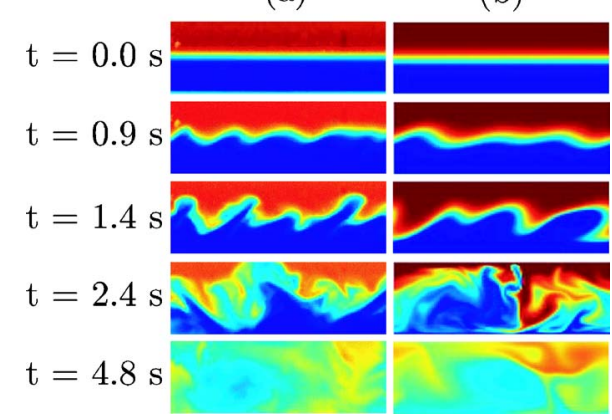

FIG. 36. (Color in online edition) Mixing due to a high- $\mathrm{Ra}_{e}$ electrohydrodynamic instability. When an electric field is applied along a channel containing solutions of different conductivities flowing side by side, charge separation at the interface introduces non-neutral fluid elements. When forcing is strong enough that the interface cannot relax by diffusion, the nonneutral fluid elements are driven by the field and amplify perturbations to cause instability. (a) An experimental time series; (b) corresponding simulations (Lin et al., 2004). Figure courtesy of J. Santiago.

ductivity of a solution depends on the local concentration of charge-carrying ions, which evolve via diffusion, advection, or due to electric forces.

As with buoyancy-driven flows, the electrohydrodynamic (EHD) fluid velocity is not known at the outset, but depends on the distribution of charges, conductivities, and fields. By analogy with buoyancy-driven flows, however, one can imagine that diffusion can outcompete slow flows so that the system evolves smoothly. On the other hand, convection or forced motion may be stronger than diffusion, in which case positive feedback and instabilities can occur.

Continuing with this analogy, one can derive electrical Grashof and Rayleigh numbers (Baygents and Baldessari, 1998; Lin et al., 2004). An EHD velocity scale is obtained under the assumption that charges separate until the fields they set up is of order the applied field $E_{0}$, giving a charge density scale $\rho_{e} \sim \epsilon_{w} E_{0} / L_{0}$. The electrical force on the fluid $f_{E}=\rho_{e} E_{0} \sim \epsilon_{w} E_{0}^{2} / L_{0}$ is balanced by viscous forces $f_{\nu} \sim \eta U / L_{0}^{2}$, giving an EHD velocity scale

$$
U_{\mathrm{EHD}} \sim \frac{\epsilon_{w} E_{0}^{2} L_{0}}{\eta} .
$$

From this velocity scale, an electrical Rayleigh number

$$
\mathrm{Ra}_{e}=\frac{U_{\mathrm{EHD}} L_{0}}{D}=\frac{\epsilon_{w} E_{0}^{2} L_{0}^{2}}{\eta D}
$$

and electrical Grashof number

$$
\mathrm{Gr}_{e}=\frac{U_{\mathrm{EHD}} L_{0}}{\nu}=\frac{\epsilon_{w} E_{0}^{2} L_{0}^{2}}{\eta \nu}
$$

follow (Baygents and Baldessari, 1998; Lin et al., 2004).

Recently, Santiago and co-workers have exploited conductivity gradients in microfluidic devices to various ends. Figure 36 demonstrates the rapid electrokinetic mixing that occurs when an EHD instability is driven
(Oddy et al., 2001). They examined this instability experimentally, analytically, and numerically (Lin et al., 2004; Chen et al., 2005), in the context of related EHD instabilities in lower-conductivity solvents (Melcher and Schwarz, 1968; Melcher and Taylor, 1969; Hoburg and Melcher, 1976, 1977; Saville, 1997; Baygents and Baldessari, 1998). Controlling and avoiding this EHD instability allowed Jung et al. (2003) to achieve a thousandfold amplication of solute concentration using field-amplified sample stacking, an order of magnitude greater than previous microfluidic devices (Jacobson and Ramsey, 1995; Lichtenberg et al., 2001; Yang and Chien, 2001).

We note finally that electrical Rayleigh systems are richer in some ways than their buoyant counterparts. While the electrical and buoyant systems described above have certain similarities, important distinctions do exist. Significantly, the charged species themselves can (and do) affect the external field strongly, whereas the gravitational interaction between buoyant or dense solute molecules is incredibly small. Such systems remain largely unexplored.

\section{F. The Knudsen number: When molecules matter}

Throughout this review, we have always assumed that the fluid can be treated as a continuum. As we ponder and probe fluids on ever smaller length scales, a natural question arises as to when the continuum approximation loses its validity.

\section{Molecular effects in gases}

We have implicitly focused on liquids thus far, and have not explicitly discussed microfluidic gas flows. Despite their importance in numerous industrial and technological applications, such flows seem to have been largely overlooked by the physics community, owing perhaps to the overwhelming importance of liquids in biology and analytical chemistry. Various issues are introduced when the working fluid is a gas rather than a liquid (Ho and Tai, 1998; Gad-el Hak, 1999), and here we focus on two primary effects.

First, the molecular-level distinction between liquids and gases can have important ramifications for microfluidic flows. While liquid molecules are in constant collision, gas molecules move ballistically and collide only rarely. Using the kinetic theory of gases, one can calculate the mean free path between collisions to be

$$
\lambda_{f} \sim \frac{1}{n a^{2}},
$$

where $n$ is the number density of molecules with radius $a$. For example, an ideal gas at $1 \mathrm{~atm}$ and $25^{\circ} \mathrm{C}$ has a mean free path $\lambda_{f} \sim 70 \mathrm{~nm}$ that increases at lower pressures or higher temperatures.

As device dimensions get smaller, the mean free path occupies an increasingly significant portion of the flowand thus plays an increasingly important role. The Knudsen number 


$$
\mathrm{Kn}=\frac{\lambda_{f}}{L}
$$

expresses the ratio of the mean free path (the length scale on which molecules matter) to a macroscopic length scale $L$. The latter is typically a length scale representative of the device, but could also be given by the length scale for temperature, pressure, or density gradients.

Noncontinuum effects play an increasing role as $\mathrm{Kn}$ increases. Roughly speaking, molecules located farther than $\lambda_{f}$ from a solid wall do not see the wall, whereas closer molecules can collide with the wall rather than other molecules. This implies that the fluid behaves like a continuum up to a distance $\lambda_{f}$ from the wall, and influences the boundary conditions obeyed by the fluid. Maxwell (1879), at the suggestion of a referee, predicted the no-slip boundary condition to be violated for diffuse wall collisions, yielding instead the slip condition

$$
\mathbf{u}_{0}=\left.\beta \frac{d \mathbf{u}}{d n}\right|_{0},
$$

where $\beta$ is a slip length of order $\lambda_{f}$. This represents the leading-order correction due to finite $\mathrm{Kn}$; second-order effects may play a role as $\mathrm{Kn}$ increases (Arkilic et al., 2001; Maurer et al., 2003).

Second, the density of a gas typically depends much more strongly on temperature and pressure than that of a liquid. Therefore, compressibility can play a much more important role in microfluidic gas flows, particularly when significant differences in $T$ or $p$ exist. Both fluid density and mean free path $\lambda_{f}$ are affected, and Kn can vary appreciably along the channel. Three distinct $\mathrm{Kn}$ regimes have been measured in pressure-driven flow: $\mathrm{Kn} \ll 1$, where the gas behaves as a no-slip fluid, $\mathrm{Kn} \sim 1$, where the gas behaves as a continuum but slips at the boundaries, and $\mathrm{Kn} \gg 1$, where the continuum approximation breaks down completely (Gad-el Hak, 1999).

\section{Fluid slip over nonwetting surfaces}

Liquid molecules, on the other hand, are in constant contact, resulting in significantly higher incompressibility and making the concept of $\lambda_{f}$ less meaningful. As such, noncontinuum effects appear to play a role only when the fluids themselves are confined to molecular length scales. Various experiments indicate that water and other liquids behave as continuum fluids until they are confined to a few (e.g., of order 10) molecular layers [e.g., Israelachvili (1986), Gee et al. (1990), and Raviv et al. (2001)].

Recently, however, measurements have indicated that the no-slip boundary condition long assumed to hold for liquids may be violated (Vinogradova, 1999; Granick et al., 2003). A variety of experimental techniques have been used to probe the solid-liquid boundary condition for various liquids: drainage forces were measured using the surface force apparatus (Horn et al., 2000; Baudry et al., 2001; Zhu and Granick, 2001, 2002a, 2002b; Cottin-
Bizonne et al., 2002) and colloid-tipped atomic force microscope cantilevers (Craig et al., 2001; Bonaccurso et al., 2002; Sun et al., 2002; Neto et al., 2003; Vinogradova and Yakubov, 2003), pressure and/or flow rate relations were measured in capillaries (Schnell, 1956; Churaev et al., 1984; Watanabe et al., 1999; Cheng and Giordano, 2002; Choi et al., 2003) and through nanopores (Cheikh and Koper, 2003), fluid velocity profiles were measured using micron-resolution particle image velocimetry (Tretheway and Meinhart, 2002), double-focus fluorescence cross correlation (Lumma et al., 2003), and fluorescence recovery after photobleaching (Pit et al., 2000). A generally (but not universally) consistent picture has emerged that wetting (hydrophilic) surfaces obey the no-slip boundary condition as expected, whereas clean nonwetting (hydrophobic) surfaces exhibit apparent slip-often to a surprisingly large extent. In fact, apparent slip lengths on the order of microns were measured-about a thousand times longer than the molecular size or mean free path one might expect. Some reports indicate a slip length that is shear-rate dependent, suggesting a yieldstress slip boundary condition (Spikes and Granick, 2003).

A fundamental question that arises is whether fluid truly slips over solid surfaces, invalidating the no-slip boundary condition, or whether the experiments reflect an apparent slip that arises from surface inhomogeneities or a complex interface with additional physics. Molecular-dynamics simulations, whose physical ingredients are known, have indeed shown fluid to slip (albeit with much shorter slip length), particularly when the attraction between molecules is stronger than the wall/ molecule attraction (Thompson and Robbins, 1990; Thompson and Troian, 1997; Barrat and Bocquet, 1999a, 1999b; Cieplak et al., 2001; Cottin-Bizonne et al., 2003). Although the shear rates employed in such simulations are much higher than in experiments, the reported slip varies linearly with shear rate, suggesting linear response behavior and a constant slip length.

Various explanations have been proposed to account for apparent slip effects (Vinogradova, 1999). A perfectly slipping but rough surface obeys a macroscopic boundary condition with slip length of order the roughness length scale (Richardson, 1973; Jansons, 1988). From this standpoint, any macroscopic slip length (real or apparent) will be confined to the roughness scale. Indeed, measured slip does decrease as surfaces are made smoother (Zhu and Granick, 2002a; Bonaccurso et al., 2003). However, even nonwetting, molecularly smooth surfaces have exhibited large apparent slip lengths. A gas layer at the interface, which would lubricate and alter the fluid flow, has been invoked for an explanation (Ruckenstein and Rajora, 1983; Ruckenstein and Churaev, 1991; Vinogradova, 1995, 1996; de Gennes, 2002; Andrienko et al., 2003; Lauga and Stone, 2003; Yang et al., 2003; Lauga and Brenner, 2004; Tretheway and Meinhart, 2004). The idea is that dissolved gas molecules are drawn out of solution to nucleate a gas layer at the solid-liquid interface and lower the surface energy (Lum et al., 1999; de Gennes, 2002; Attard, 2003; Gran- 
ick et al., 2003). This hypothesis is bolstered by the observation of nanobubbles using tapping-mode atomic force microscopy (Ishida et al., 2000; Tyrrell and Attard, 2001, 2002), and further supported by the observation that apparent slip decreases when the fluid is degassed (Boehnke et al., 1999; Granick et al., 2003).

The no-slip boundary condition never enjoyed solid theoretical backing, but rather was accepted due to its apparent experimental success (Goldstein, 1938). While no consensus has been reached on the physical origin of the measured slip, its effects will be felt as microfluidic devices are made ever smaller. An understanding of the apparent slip might allow surfaces to be designed specifically to slip, e.g., to reduce hydrodynamic resistance and dispersion (Cottin-Bizonne et al., 2003; Granick et $a l ., 2003)$. In this case, an analogous Knudsen number

$$
\mathrm{Kn}=\frac{\beta}{L}
$$

will be significant in the description of such flows.

\section{DRIVING FLOWS AT THE BOUNDARIES}

Owing to the small dimensions of microfluidic devices and to the long-range nature of viscous flows, boundaries are never very far in microfluidics. Therefore, techniques that exploit boundary effects can be quite effective in microfluidic manipulation. In what follows, we describe three such phenomena: electrokinetic effects, acoustic streaming, and fluid-structure interactions.

\section{A. Electrokinetic effects}

Of all nonmechanical fluid manipulation techniques in microfluidic contexts, electrokinetic techniques are almost certainly the most popular. Historically, early microfluidic devices were designed for capillary electrophoretic separations, and it was natural to use electroosmotic flow (EOF) to pump as well as to separate. [See Ghosal (2004) for a review of the physics of EOF.] EOF in perfectly uniform, straight channels has a flat velocity profile, implying less convective dispersion than for pressure-driven flow. Note, however, that Taylor dispersion reduces convective dispersion-so that in sufficiently thin channels (for which Pe $\leqslant 1$ ), tracer distributions in EOF and pressure-driven flow will evolve similarly. Finally, EOF velocities do not depend on the microchannel dimensions, in striking contrast with pressure-driven flows, whose velocity decreases with the second power of channel size.

However, electrokinetic techniques exhibit significant drawbacks. Electro-osmotic flows are not very robust, as they depend sensitively on the physicochemical properties of the solution and channel walls. Electro-osmotic flows depend on the walls' charge density, which varies with solution $p \mathrm{H}$, ionic strength, and (uncontrollably) when solute molecules adsorb onto the walls. Inhomogeneities in surface charge density-intended or notgive rise to stray pressure gradients that disrupt the plug
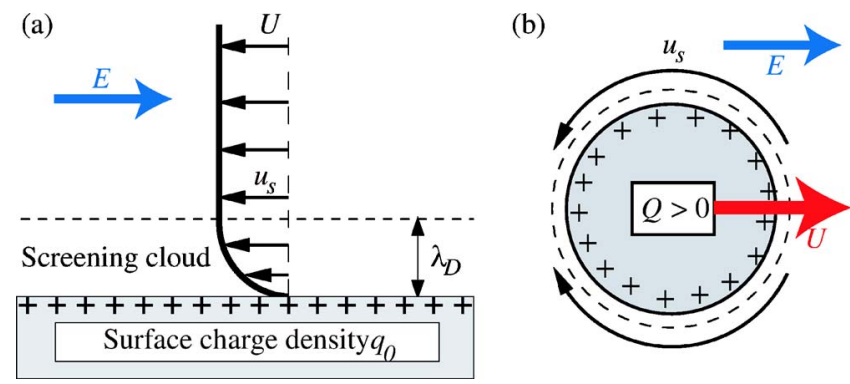

FIG. 37. (Color in online edition) (a) An ionic screening cloud of width $\sim \lambda_{D}$ forms around a charged solid surface in an electrolytic solution. An external electric field forces the mobile ions in the screening cloud to result in electro-osmotic flow [Eq. (46)]. (b) Under an applied electric field, a freely suspended charged particle in an electrolyte moves via electrophoresis, with velocity equal in magnitude and opposite in direction to Eq. (46). Reprinted with permission from Squires and Bazant, 2004. (C2004, Cambridge University Press.

nature of the flow. Large voltages are typically required, reducing the portability of the device. Electrochemical reactions must occur at electrodes in order to maintain an electric field in solution, which give rise to a host of adverse effects, including metallic ion injection, water electrolysis and its associated bubble formation, and the introduction of $p \mathrm{H}$ or solute gradients.

Here, we focus on the basic physics of electrokinetics, and explore recent variants on classic techniques. More detailed accounts of the fundamentals are given in textbooks and reviews (Levich, 1962; Saville, 1977; Anderson, 1989; Russel et al., 1989; Lyklema, 1991; Probstein, 1994; Hunter, 2000; Ghosal, 2004).

\section{Electrostatics in ionic solutions}

Aqueous solutions are conductive due to the ubiquitous presence of dissolved ions (e.g., from dissolved salts, ionic groups on surfaces, or dissociated water molecules). Solid-liquid interfaces tend to develop surface charge, which attracts oppositely charged counterions and repels similarly charged co-ions. The resulting ionic double layer screens the surface charge over a characteristic Debye length $\lambda_{D}$. With the exception of these charged double layers, the fluid is charge neutral.

The diffuse part of the double layer [Fig. 37(a)] is established when diffusive transport tending to smooth ion gradients balances electrostatic transport driving counterions towards the interface. [A compact layer also exists (Russel et al., 1989; Lyklema, 1991; Hunter, 2000), but we neglect it here.] We describe the diffuse layer for a simple electrolyte of monovalent ions; results for multiple, multivalent ions follow naturally and can be found in the above-mentioned textbooks.

Ions in solution with number density $n_{ \pm}$and charge $\pm e$ set up an electrostatic field $\phi$ obeying Poisson's equation, 
TABLE IV. Ion densities and screening lengths for aqueous electrolytic solutions of varying salt concentration at $20^{\circ} \mathrm{C}$.

\begin{tabular}{|c|c|c|}
\hline \multicolumn{3}{|c|}{ Properties of aqueous electrolytic solutions } \\
\hline Concentration & Ion density (ions $/ \mathrm{nm}^{3}$ ) & $\lambda_{D}$ \\
\hline $1 M$ & 0.6 & $0.3 \mathrm{~nm}$ \\
\hline $0.1 M$ & 0.06 & $1 \mathrm{~nm}$ \\
\hline $1 \mathrm{~m} M$ & $6 \times 10^{-4}$ & $10 \mathrm{~nm}$ \\
\hline $0.1 \mu M$ (pure, $p \mathrm{H} 7$ ) & $6 \times 10^{-8}$ & $1 \mu \mathrm{m}$ \\
\hline
\end{tabular}

$$
\nabla^{2} \phi=-\frac{\rho_{e}}{\epsilon_{w}}=-\frac{e\left(n_{+}-n_{-}\right)}{\epsilon_{w}},
$$

where $\epsilon_{w}$ is the permittivity of the solution. Due to their small size, the ions are highly mobile and respond quickly to the local electric field with potential $\psi$. In equilibrium, each ion is statistically distributed according to the Boltzmann distribution

$$
n_{ \pm}=n_{0} \exp \left(\mp \frac{e \psi}{k_{B} T}\right)
$$

where $n_{0}$ is the bulk concentration of ions. The fields are related by the mean-field assumption: the electrostatic potential $\phi$ set up by the ions [Eq. (39)] is assumed to be the same as the field $\psi$ to which they respond [Eq. (40)]. Combining Eqs. (39) and (40), we arrive at the nonlinear Poisson-Boltzmann equations

$$
\nabla^{2}\left(\frac{e \phi}{k_{B} T}\right)=k^{2} \sinh \left(\frac{e \phi}{k_{B} T}\right),
$$

which can be linearized for small potentials $\left(\phi \ll k_{B} T / e\right.$ $\approx 26 \mathrm{mV}$ ), giving

$$
\nabla^{2} \phi=\kappa^{2} \phi .
$$

Here $\lambda_{D}=\kappa^{-1}$ is the screening length,

$$
\kappa^{-1} \equiv \lambda_{D}=\left(\frac{\epsilon_{w} k_{B} T}{2 n_{0} e^{2}}\right)^{1 / 2} \equiv\left(8 \pi n_{0} \lambda_{B}\right)^{-1 / 2}
$$

where $\lambda_{B}=e^{2} / 4 \pi \epsilon_{w} k_{B} T(\approx 0.7 \mathrm{~nm}$ in water $)$ is the Bjerrum length, reflecting the distance that must be maintained between oppositely charged ions to prevent aggregation. Water dissolves salts so well because its small, polar molecules form hydration shells around ions that enforce this separation; nonpolar solvents do not, and thus have much lower conductivities (Morrison, 1993). Table IV provides typical screening lengths. Notably, even pure $p \mathrm{H}-7$ water has only a $1-\mu \mathrm{m}$ screening length. Typical aqueous screening lengths are $\sim 1 \mathrm{~nm}-$ much smaller than typical device dimensions.

A charged planar surface has a screening cloud obeying Eq. (42), giving $\phi \sim \zeta e^{-\kappa y}$. The potential drop across the charge cloud, called the zeta potential $\zeta$, is related to the surface charge density $q_{0}$ via

$$
\zeta=\frac{q_{0}}{\epsilon_{w} \kappa}
$$

for weak potentials. Nonlinear screening for strong potentials gives a double-layer capacitance that depends exponentially on $\zeta$ (Russel et al., 1989; Lyklema, 1991; Hunter, 2000).

\section{Electrokinetics: Electro-osmosis and electrophoresis}

When an external electric field $E_{\|}$is applied along a charged surface, the nonzero charge density in the diffuse layer gives rise to an electric body force $\mathbf{f}=\rho_{e} E_{\|} \hat{\mathbf{z}}$, also tangent to the surface. In this case, the Stokes equations [Eq. (2)] can be integrated to give

$$
u=\frac{\epsilon_{w} \zeta}{\eta} E_{\|}\left(1-e^{-\kappa y}\right),
$$

representing a fluid velocity that exponentially approaches Smoluchowski's constant slip velocity,

$$
u_{s}=\frac{\epsilon_{w} \zeta}{\eta} E_{\|},
$$

outside of the diffuse layer. When channel walls comprise the solid-fluid interface, electro-osmotic flow (EOF) is driven [Fig. 37(a)] with a velocity that varies linearly with the applied field [Eq. (46)].

When the charged solid-fluid interface is a freely suspended particle, electro-osmotic slip causes the particle itself to move electrophoretically [Fig. 37(b)] with velocity

$$
u=\frac{\epsilon_{w} \zeta}{\eta} E_{\|} \equiv \mu_{E} E_{\|}
$$

Several rather surprising results hold in the limit where the double-layer $\lambda_{D}$ is thin compared to the particle size: the electrophoretic mobility $\mu_{E}$ for an object with fixed $\zeta$ is independent of particle size, shape, or number; multiple particles of the same zeta potential (but possibly different shape and size) experience identically zero interaction; and the fluid flow is parallel everywhere to electric field lines (Morrison, 1970; Anderson, 1989). Finally, the electrophoretic mobility of a particle is independent of its dielectric constant (O'Brien and White, 1978).

\section{Recent variations on classical electrokinetic techniques}

We have discussed the classic electrokinetic picture, in which homogeneous surfaces possess a constant surface charge-zeta potential. Interesting and useful phenomena can arise in systems with inhomogeneous zeta potentials. Here we explore three variations on this theme: (i) systems with patterned surface charge, (ii) systems whose surface charge can be actively controlled, and (iii) nonlinear induced-charge electrokinetic flows that occur when applied fields act on the charge clouds that they induce around polarizable surfaces. 
(a)
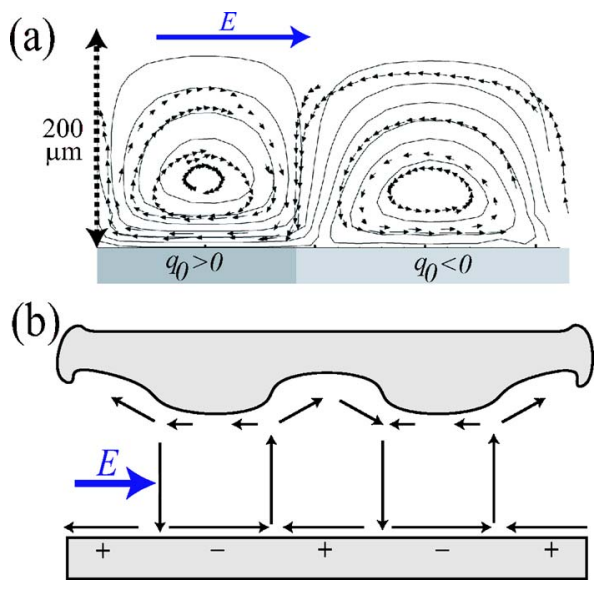

FIG. 38. (Color in online edition) Electro-osmotic flow over inhomogeneously charged surfaces. (a) Tracer particle trajectories demonstrate the cellular EOF flow over a surface with alternating surface charge density. Adapted with permission from Stroock et al., 2000. (b) Patterning both surface charge density and surface shape allows a net flow to be driven even for a surface with no net charge. Reprinted with permission from Ajdari, 1995.

\section{a. Patterned surfaces with nonuniform charge}

The above discussion (and most studies in general) assumes constant $\zeta$. Somewhat recently, electrokinetic effects involving inhomogeneous $\zeta$ have been investigated, with sometimes surprising results. Teubner (1982) considered electro-osmotic flow over surfaces with inhomogeneous zeta potential, but did not focus on its peculiarities. Anderson and Idol (1985) highlighted a rather counterintuitive result: a particle's charge cannot be determined from its electrophoretic mobility alone as is often assumed. Anderson (1985) studied EOF through an inhomogeneously charged channel and found that while the average flow is proportional to the average $\zeta$, the flow itself is highly inhomogeneous and contains recirculating rolls. Related work explored the electrophoresis of inhomogeneously charged particles of various geometries (Fair and Anderson, 1989, 1990, 1992; Fair, 1990; Solomentsev and Anderson, 1995; Long and Ajdari, 1996, 1998). For example, Long and Ajdari (1998) found cylindrical shapes with special cross section and charge distributions whose electrophoretic velocity is always perpendicular to the applied field.

Ajdari (1995) furthered these ideas and explored their use in microfluidic systems, with an emphasis on the rich phenomena that occur when spatial symmetries are broken. For example, a net electro-osmotic pumping can occur over a surface with no average charge when both the shape and the surface charge density are allowed to vary [Fig. 38(b)], and electro-osmotic flows can be driven either transverse or parallel to the applied field by breaking appropriate charge and shape symmetries (Ajdari, 1995, 1996, 2002; Stroock et al., 2000; Gitlin et al., 2003). An advantage to transverse electrokinetic pumping is that modest potentials applied across narrow channels give strong fields and flows (Gitlin et al., 2003).
Related work includes investigations into the threedimensional EOF generated by nonuniform $\zeta$ distributions (Long et al., 1999; Erickson and Li, 2002b; Ghosal, 2002b), EOF in inhomogeneous microchannels with finite double layer (Ren and $\mathrm{Li}, 2001$ ), and EOF in a microchannel containing two different fluids and thus a step change in $\zeta$ that displace one another (Herr et al., 2000; Ren et al., 2001). Microchannels with nonuniform $\zeta$ or nonplanar surface topography provide a natural way to mix fluids (Stroock et al., 2000; Erickson and Li, 2002a; Johnson and Locascio, 2002; Johnson et al., 2002; Qian and Bau, 2002). However, nonuniform zeta potentials (whether intentional or not) and channel turns induce additional dispersion and band broadening in capillary electrophoresis (Long et al., 1999; Ghosal, 2002a, 2002b, 2003, 2004). Local $\zeta$ variations (Johnson et al., 2001; Henry et al., 2002; Qiao and Aluru, 2003) or special channel profiles (Culbertson et al., 2000; Griffiths and Nilson, 2000, 2002; Paegel et al., 2000; Zubritsky, 2000; Dutta and Leighton, 2001, 2002; Molho et al., 2001; Fiechtner and Cummings, 2003, 2004) have been designed to minimize such effects.

\section{b. Active control of surface charge}

Because conventional electro-osmotic flow varies linearly with the zeta potential of the wall, actively modifying the zeta potential of the wall allows active control of the electro-osmotic flow. Such ideas were applied for EOF control: by analogy with the microelectronic fieldeffect transistor (FET), microfluidic flow FETs have been developed (Ghowsi and Gale, 1991; Gajar and Geis, 1992; Schasfoort et al., 1999; Buch et al., 2001). Flow FETs employ an additional electrode embedded behind the insulating channel walls; maintaining a nonzero potential difference between this auxillary electrode and the fluid modifies the effective zeta potential, which in turn modifies the EOF. Another method for controlling surface charge densities involves coated walls with $\mathrm{TiO}_{2}$, whose $\zeta$ changes under UV light (Moorthy et al., 2001).

\section{c. Induced-charge electrokinetic phenomena}

Above, we have discussed the electrokinetics of charges with inhomogeneous zeta potentials due to patterned surfaces or to active charge control. In this final section, we discuss cases where such inhomogeneities naturally occur due to charge clouds that are induced around conducting or polarizable surfaces. These give rise to various nonlinear electrokinetic phenomena that have been discovered and explored in recent years, including "ac electro-osmosis" (Ramos et al., 1999; Ajdari, 2000), ac colloidal self-assembly near electrodes (Trau et al., 1997; Yeh et al., 1997; Gong et al., 2002, 2003; Nadal, Argoul, Hanusse, et al., 2002; Ristenpart et al., 2003), and induced-charge electrokinetic phenomena around conducting colloids and posts (Murtsovkin, 1996; Bazant and Squires, 2004; Squires and Bazant, 2004). The ingredient that unifies all of these phenomena is a charge 
cloud that is induced by the external field, and subsequently driven by that same field, so that $U \sim E_{0}^{2}$. The term induced-charge electrokinetic (ICEK) phenomena emphasizes the central role played by the induced charges. Two broad classes of ICEK flows have been studied: (i) those whose inducing surfaces are the electrodes themselves, and (ii) those whose inducing surface is independent of the electrodes. The former rely on transient fields and charge clouds, and require ac fields of a suitable frequency, whereas the latter can persist with either dc or ac driving.

ICEK phenomena were initially explored in the colloid community. Levich (1962) considered the charge cloud induced around a conducting metal sphere in a constant electric field in order to demonstrate that the electrophoretic mobility of such a particle is the same as if it were an insulator. The actual flows around such colloids are more difficult to measure than electrophoretic velocities, and are of secondary importance to colloid science. As a result, the flows received little attention until Gamayunov, Murtsovkin, Dukhin, and others investigated them theoretically (Dukhin, 1986; Dukhin and Murtsovkin, 1986; Gamayunov et al., 1986; Murtsovkin, 1996) and experimentally (Murtsovkin and Mantrov, 1991; Gamayunov et al., 1992).

Similar ideas have recently been developed for microfluidic devices. We illustrate with perhaps the simplest example of induced-charge electrokinetic phenomena, illustrated in Fig. 39 (Murtsovkin, 1996; Bazant and Squires, 2004; Squires and Bazant, 2004). An electric field that is suddenly applied across an electrolytic solution containing a conducting post initially intersects the post at right angles to satisfy the equipotential condition, as in Fig. 39(a). However, mobile ions in solution are driven along field lines and accumulate at the liquidsolid boundary, causing the field itself to evolve. A dipolar-induced charge cloud develops-negatively charged on one side, and positively on the other-that reaches steady state when no field lines penetrate the charge cloud, as in Fig. 39(b). In steady state, the induced zeta potential is dipolar, with magnitude $\zeta_{i}$ $\approx E_{0} R$ representing the potential drop of the field $E_{0}$ over the post size $R$. Using Eq. (46), an induced-charge electro-osmotic slip velocity is established with magnitude

$$
U_{\mathrm{ICEO}} \approx \frac{\epsilon_{w} E_{0}^{2} R}{\eta} .
$$

Not surprisingly, the ICEO velocity scale is the same as the EHD velocity scale [Eq. (32)]. The ICEO flow is generically quadrupolar, with fluid that is drawn towards the body along the axis of the field, and is ejected from the equator [Fig. 39(c)]. Such flows have been seen in microfluidic (Levitan et al., 2005) and colloidal (Gamayunov et al., 1992) experiments.

An important characteristic of ICEK flows is that they persist in ac fields, unlike their classic fixed-charge counterparts which average to zero. Reversing the applied field reverses the sign of the induced charge cloud, leav-
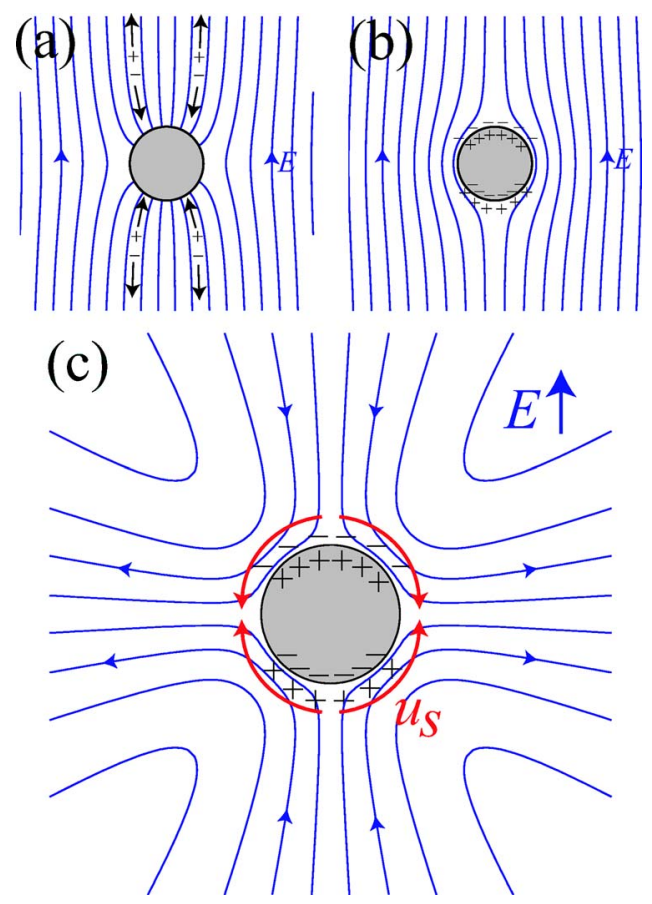

FIG. 39. (Color in online edition) (a) A suddenly applied electric field intersects a conducting cylinder at right angles to maintain an equipotential surface. A current is set up, however, since mobile ions are driven along the field. These ions accumulate in an induced charge double layer adjacent to the conducting surface. (b) Steady state is achieved when the induced double layer has developed sufficiently that no field lines penetrate the double layer. (c) The steady-state ICEO flow established around a conducting cylinder. The quadrupolar nature of the electro-osmotic slip velocity can be understood from (b), as the product of a (dipolar) field driving a (dipolar) induced charge cloud. This flow persists in an ac field, since reversing the field direction reverses the sign of the induced charge. Therefore the flow, which involves the product of the two, is unchanged. Reprinted with permission from Squires and Bazant, 2004. (C2004, Cambridge University Press.

ing the resulting flow (which depends on the product of the two) unchanged. ac electrokinetic effects circumvent many of the problems that plague dc electrokinetics, arising from the electrochemical reactions required to maintain a steady electric field. In a recent ICEK variant, Lastochkin et al. (2004) drive such reactions deliberately (which depend exponentially on voltage) and achieve 10 times stronger flows than with the capacitive, ideally polarized electrode techniques described above.

ac fields can be established without electrochemical reactions, so long as the frequency is low enough that induced charge clouds have time to form, but fast enough that electrodes and the fields they establish are not screened. The time scale required for an induced charge cloud to form around a conducting body is given by

$$
\tau_{c} \approx \frac{\lambda_{D} R}{D_{i}}
$$

where $D_{i}$ is the diffusivity of the ions. This represents the $R C$ time for an equivalent circuit consisting of a 

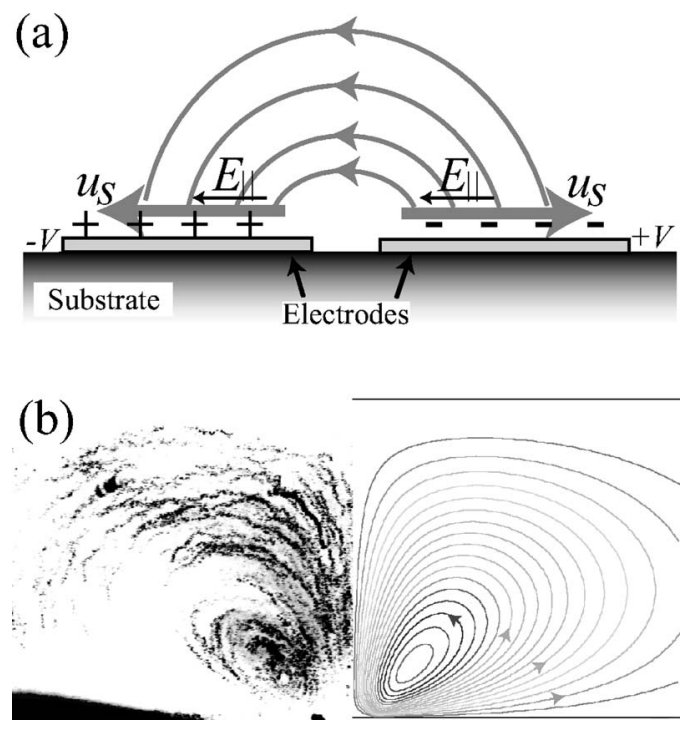

FIG. 40. ac electro-osmosis over coplanar electrodes. (a) When co-planar electrodes are driven near a particular frequency, an inhomogeneous charge cloud is induced over each electrode, which both modifies the external field and is driven by it. (b) The rectified, steady ac electro-osmotic flow, with measured streaklines on the left and calculated streamlines on the right. If the driving frequency is too small, charge clouds fully screen each electrode, and no external field exists to drive the flow. On the other hand, if the frequency is too fast, induced charge clouds do not have time to form. Therefore, ac electro-osmosis is strongest at a given (charging) frequency. Adapted with permission from from Green et al., 2002.

charge cloud capacitor and a bulk resistor (MacDonald, 1970; Simonov and Shilov, 1977; Bazant and Squires, 2004; Squires and Bazant, 2004). Similarly, the time scale over which the electrodes themselves are screened is given by $\tau_{e} \approx \lambda_{D} L / D_{i}$, where $L$ is the separation between electrodes (Ajdari, 2000; Gonzalez et al., 2000; Hollingsworth and Saville, 2003; Bazant et al., 2004). Thus, ICEK applications allow closely spaced electrodes to be used, so that higher fields can be established using low applied voltages.

This picture allows an easier understanding of the first work on microfluidic induced-charge electrokinetic phenomena (Ramos et al., 1999; Ajdari, 2000). In these systems, induced charge clouds form inhomogeneously around the electrodes themselves, so that the electrode screening time $\tau_{\mathrm{el}}$ and the charge cloud formation time $\tau_{c}$ coincide (Ramos et al., 1999; Ajdari, 2000; Gonzalez et al., 2000). Transient fields drive transient charge clouds to establish these flows, and thus are limited to frequencies around $\omega \sim \tau_{\mathrm{el}}^{-1}$. Three such systems have been investigated: (i) a pair of coplanar electrodes deposited on a single channel wall as in Fig. 40 (Ramos et al., 1999; Gonzalez et al., 2000; Green, Ramos, Gonzalez, et al., 2000; Green et al., 2002); (ii) an asymmetric array of coplanar electrodes embedded in a channel wall (Ajdari, 2000; Brown et al., 2001; Studer et al., 2002; Mpholo et al., 2003; Ramos et al., 2003); and (iii) a dielectric "stripe" deposited on an electrode (Nadal, Argoul, Kes- tener, et al., 2002). The symmetric systems produce steady, counter-rotating fluid rolls (Ramos et al., 1999; Gonzalez et al., 2000; Green, Ramos, Gonzalez, et al., 2000; Green et al., 2002; Nadal, Argoul, Kestener, et al., 2002). Asymmetric electrodes pump fluid in the direction of broken symmetry (Ajdari, 2000; Ramos et al., 2003), as was verified experimentally (Brown et al., 2001; Studer et al., 2002; Mpholo et al., 2003). A pump exploiting this effect establishes flow velocities of $450 \mu \mathrm{m} / \mathrm{s}$ using 2-V, 10-kHz rms applied potentials (Mpholo et al., 2003).

ICEK phenomena are not limited to conducting surfaces, as induced charge clouds form around any polarizable (dielectric) surface, although they are strongest around clean conducting surfaces (Squires and Bazant, 2004). Thamida and Chang (2002) observed a nonlinear electrokinetic jet directed away from a corner that can be understood with the above picture. A variety of physical processes enrich such systems: Takhistov et al. (2003) observe vortices at sharp channel corners, and argue that they occur due to electrolyte depletion under the strong field near the corner. Ben and Chang (2002) and Lastochkin et al. (2004) have investigated related systems under higher applied potentials, in which electrochemical Faradaic reactions occur at electrodes, yielding stronger microfluidic flows.

\section{B. Acoustic streaming}

Little explored thus far in microfluidics, acoustic streaming represents one of very few inertial phenomena that may actually play a significant role in microfluidic devices. As discussed in Sec. II.A, small feature sizes typically prevent flow velocities from being high enough to yield high numbers. High-frequency acoustic waves, however, can circumvent such difficulties. At first, periodic wave motion might seem to be of little use for fluid manipulation. However, the inertial nonlinearity can rectify oscillatory fluid motion to give a time-averaged flow called steady or acoustic streaming, as shown by Rayleigh (1884). A basic introduction is given in Chap. 5.13 of Batchelor (2001), more comprehensive treatments can be found in Chap. 8.4 of Lighthill (2001) and Lighthill (1978), and detailed reviews are given by Stuart (1963), Nyborg (1998), and Riley (2001). Here, we discuss three broad classes of steady streaming: quartz wind, boundary-induced streaming, and cavitation microstreaming.

\section{Quartz wind}

The simplest manifestion of acoustic streaming is the quartz wind, named for the wind observed to blow away from oscillating quartz crystals. Dissipation causes acoustic waves to decay over an attenuation length $\alpha^{-1}(\omega)$, resulting in an oscillatory velocity field $u_{\omega}$ $\sim U_{0} e^{-\alpha z} e^{i \omega t} \hat{z}$ with zero time average. However, the oscillation amplitude of a fluid particle decreases in one half cycle as it moves away from the source; conversely, its amplitude increases as it moves toward the source. 
Oscillating fluid particles thus constantly impart momentum to the fluid, driving a secondary streaming flow away from the acoustic source (Lighthill, 1978). This rectified forcing comes from the nonlinear inertial term that we have so far neglected. Time averaging Eq. (1) yields a steady body force

$$
\mathbf{f}_{Q W}=-\rho\left\langle\mathbf{u}_{\omega} \cdot \nabla \mathbf{u}_{\omega}\right\rangle \sim \rho \alpha U_{0}^{2} e^{-2 \alpha z} \hat{z},
$$

which drives the wind felt in front of stereo loudspeakers.

The quartz wind can be used to drive microfluidic flows by sending an acoustic plane wave along a microchannel of length $L \gtrsim \alpha^{-1}$. The body force gives a pressure drop $\Delta P_{Q W}=\int f_{Q W} d z \sim \rho U_{0}^{2}$ that drives a Poiseuille flow with flow rate

$$
Q_{Q W} \sim \frac{\rho U_{0}^{2} w^{4}}{\eta L}
$$

which suffers from the $w^{4}$ scaling typical of pressuredriven flows. For appreciable forcing, the channel length should have dimensions comparable to or larger than the attenuation length $\alpha^{-1}$. Since $\alpha^{-1} \approx 8.3 \mathrm{~mm}$ in water at $50 \mathrm{MHz}$ and decreases like $\omega^{-2}$ (Rife et al., 2000), a natural size limit is imposed on quartz-wind-driven microfluidic devices, which are typically centimeter sized (Zhu and Kim, 1998; Rife et al., 2000; Yang et al., 2000, 2001).

\section{Boundary-induced streaming}

While the quartz wind occurs in the bulk of the fluid, steady streaming flows occur around solid boundaries as well, with an amplitude that is (remarkably) independent of viscosity.

An oscillatory flow (with amplitude $U_{0}$ ) over a solid surface must vanish on the surface due to the no-slip condition. In unsteady viscous flows, vorticity generated at the wall spreads diffusively (with diffusivity $\nu$ ), so that wall effects are confined to the distance that vorticity can diffuse in one cycle before it is annihilated. An oscillatory boundary layer (called a Stokes layer) of size

$$
\delta \sim\left(\frac{\nu}{\omega}\right)^{1 / 2}
$$

contains all of the vorticity in the flow, with irrotational flow outside of the Stokes layer.

If the amplitude of oscillatory flow $U_{0}$ above the Stokes layer varies with length scale $R$ along the boundary, the nonlinear inertial term $\rho \mathbf{u} \cdot \nabla \mathbf{u}$ rectifies the oscillatory flow to give a steady inertial forcing, as with the quartz wind. The steady inertial force $\left(f_{i} \sim \rho U_{0}^{2} / R\right)$ is balanced by steady viscous forces $f_{v} \sim \eta u_{s} / \delta^{2} \sim \rho \omega u_{s}$ that are, ironically, independent of viscosity. This results in a velocity scale for steady boundary-driven streaming,

$$
u_{s} \sim \frac{U_{0}^{2}}{\omega R} .
$$

The acoustic boundary-driven slip velocity outside the Stokes layer is given by

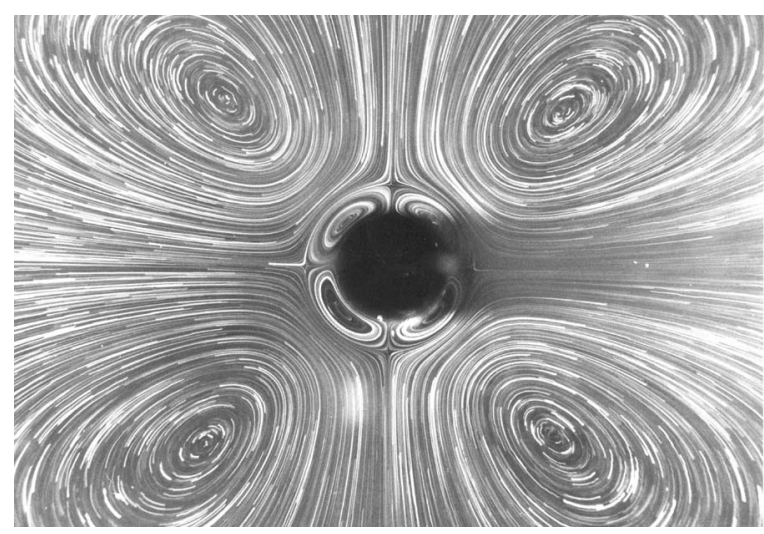

FIG. 41. Acoustic streaming around a cylinder. The inertial nonlinearity rectifies the oscillatory flow, leading to the steady streaming flow shown. Outside of the oscillatory boundary layer, the steady streaming flow is directed towards the stagnation points at the top and bottom. The streaming flow has small streaming Reynolds number $\mathrm{Re}_{s}$ and decays with distance as $r^{-1}$. Figure courtesy of Tatsuno; experiments described in Tatsuno, 1980.

$$
u_{s} \sim-\frac{3}{4 \omega} U_{0} \frac{d}{d x_{\|}} U_{0},
$$

and flows in the direction of decreasing free-stream velocity (e.g., toward stagnation points).

Boundary-induced acoustic streaming is reminiscent of electro-osmotic flow (Sec. III.A), in that body forces localized near a surface give rise to an effective slip velocity. However, EOF increases monotonically to Eq. (46), whereas streaming flows reverse direction within the Stokes layer, causing recirculating rolls (Fig. 41).

The flow outside of the Stokes layer obeys the NavierStokes equations [Eq. (1)] with a slip velocity given by Eq. (54). The external flow due to this slip velocity has an associated streaming Reynolds number,

$$
\operatorname{Re}_{s}=\frac{u_{s} R}{\nu}=\frac{U_{0}^{2}}{\omega \nu}
$$

giving two regimes of acoustic streaming, for large or small $\operatorname{Re}_{s}$ (Stuart, 1963, 1966). Early work on steady streaming flows almost universally assumed $\operatorname{Re}_{s} \ll 1$, a regime called RNW streaming (Rayleigh, 1884; Nyborg, 1953; Westervelt, 1953). In the macroscopic world, large$\mathrm{Re}_{s}$ Stuart streaming flows are more common, although more challenging theoretically. Speaking broadly, RNW streaming involves long-ranged Stokes flows, whereas Stuart streaming flows are confined to an outer boundary layer of size $\mathrm{Re}_{s}^{-1 / 2} R$, along with isolated jets and potential flows.

The free-streaming velocity can change for several reasons:

Standing acoustic waves. Historically the first theoretical treatment, Rayleigh (1884) calculated the secondary streaming due to a standing acoustic wave in a tube. As expected, flow is towards the nodes of the standing wave pattern.

Acoustic attenuation. Acoustic attenuation gives a 


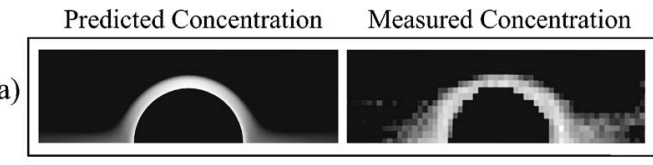

(b)

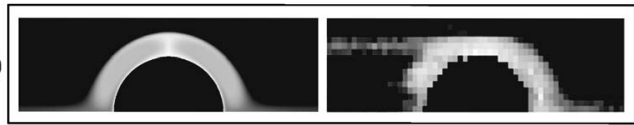

(c)

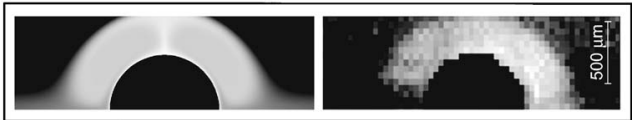

FIG. 42. Acoustic streaming and chemical capture around a microelectrode post. An electrochemical reaction releases a marker into the fluid that is captured within the Stokes layer. The left series represents theory and the right represents experiments. (a), (b), and (c) Reynolds number $\operatorname{Re}=a^{2} \omega / \eta=500$, 200 , and 100, respectively. Adapted and reprinted with permission from Lutz et al., 2003. (C2003, National Academy of Sciences.

length scale $R \sim \alpha^{-1}$ over which the free streaming velocity changes. Thus two types of streaming occur when attenuating acoustic waves travel down a channel: (i) the quartz wind drives a parabolic flow, as discussed above, and (ii) boundary-driven streaming establishes a plug flow. As channels get smaller, boundary-driven streaming dominates (Frampton et al., 2003): a local slip velocity is established with magnitude $u_{s} \sim \alpha U_{0}^{2} /$ $\omega \exp (-2 \alpha z)$, that gives an average flow rate $Q$ $\sim \alpha U_{0}^{2} w^{2} / \omega$ down a long channel, whose $w^{2}$ scaling can exceed the $w^{4}$ quartz-wind scaling [Eq. (51)] for small enough $w$.

Curved boundaries. Steady streaming occurs around vibrating spheres and cylinders or (equivalently) due to oscillatory flow past cylinders and spheres [Riley (2001), and references therein]. From Eq. (54), the streaming slip velocity (just outside the Stokes layer) is directed towards the decreasing free-stream velocity, i.e., towards the stagnation points at the two poles. The resulting flow is thus quadrupolar, and decays with distance as $r^{-1}$ for cylinders ( $r^{-2}$ for spheres). Flow reversal in the Stokes layer gives rise to a set of recirculating eddies (Fig. 41) whose size varies as $\nu \omega^{-1 / 2}$ [Eq. (52)]. Recently, such eddies have been exploited to make a microchemical trap, shown in Fig. 42, that stores chemicals released at the cylinder surface (Lutz et al., 2003).

Examples that may find use for mixing and pumping in microfluidics include streaming due to oscillatory flows in curved pipes [Fig. 43(c)] (Lyne, 1971), tapering channels (Grotberg, 1984; Gaver and Grotberg, 1986), and channels of variable cross section [Figs. 43(a) and 43(b)] (Hall, 1974). The streaming flow in a curved pipe [Fig. 43(c)] resembles Dean flow (Fig. 4), suggesting a zero-Re analog of the serpentine mixer (Liu et al., 2000) that uses acoustic waves, rather than the primary pressure-driven flow, to introduce the stirring flow. Note that the flow in Fig. 43(c) is directed opposite to Dean flow (except within the Stokes layer, where the flow is

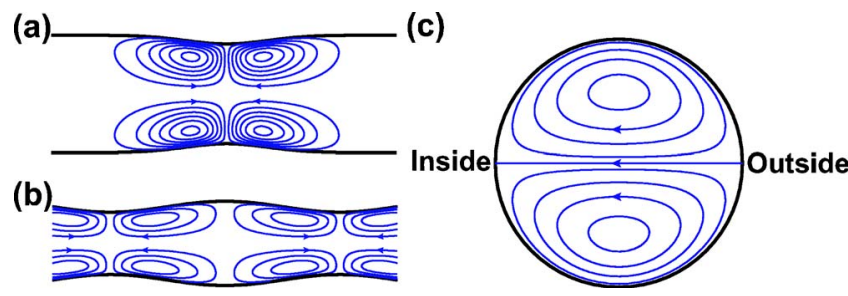

FIG. 43. (Color in online edition) (a,b) Secondary streaming eddies in an oscillatory flow that arise due to (a) a localized bump in the channel profile (Hall, 1974), and (b) a sinusoidally varying channel profile (Hall, 1974). (c) Streaming flow arising from oscillatory flow in a slightly curved channel (Lyne, 1971). Flow in the central region of the pipe resembles Dean flow (Fig. 4), but flows in the opposite direction.

centrifugally forced). In the low-frequency limit, the Stokes layer becomes larger than the pipe itself, and Dean flow is recovered.

\section{Cavitation microstreaming}

We discuss one last streaming mechanism that has been exploited for microfluidic use. Bubbles subjected to acoustic waves expand and contract as the local pressure varies. Bubble shape and fluid oscillations are rectified to give so-called cavitation microstreaming (Elder, 1959), and bubbles that both translate and pulsate give rise to a dipolar streaming flow that is longer ranged for spherical bubbles $\left(u_{s} \sim r^{-1}\right)$ than for solid spheres $\left(u_{s}\right.$ $\sim r^{-2}$ ) (Longuet-Higgins, 1998). Bubble translations and oscillations occur simultaneously when a bubble is attached to a solid wall, since volume conservation requires the bubble center to move away from the wall as it expands. The streaming flow around an attached bubble is shown in Fig. 44, and has been used to lyse vesicles (Marmottant and Hilgenfeldt, 2003) and to enhance mixing in cell capture (Liu, Yang, et al., 2002) and DNA hybridization applications (Liu, Lenigk, et al.,

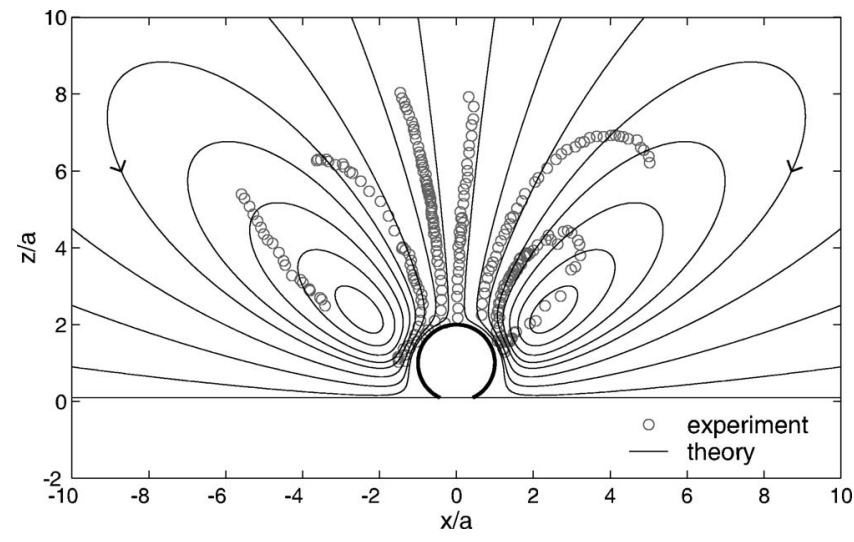

FIG. 44. Cavitation microstreaming. The streaming flow around a pulsating and translationally oscillating bubble gives shear stresses that can be used to lyse vesicles. Measured particle trajectories are shown with calculated streamlines. Reprinted with permission from Marmottant and Hilgenfeldt, 2003. (C2003, NPG. 
2003). A further rectification occurs when the bubble is held adjacent to a solid protrusion, in which case a net pumping occurs in the direction of broken symmetry (Marmottant and Hilgenfeldt, 2004).

\section{Fluid-structure interactions}

Our final example of boundary-driven flows is perhaps the simplest: moving the walls themselves in order to physically force the fluid. This strategy is practical when the walls are sufficiently compliant to allow significant deformation. Soft polymeric materials have tunable elastic moduli that are typically many orders of magnitude lower than crystalline solids like silicon, and thus have proven popular and successful for such applications (Quake and Scherer, 2000; Whitesides et al., 2001).

We discuss here two broad methods of controlling the fluid by deforming the structure that encloses it. First, externally applied pressure can deform membranes and displace fluid, creating valves and pumps. Second, active and self-regulating fluid control elements can be built with intelligent hydrogels that swell or collapse as environmental conditions change.

\section{Driving flows with soft walls}

Mechanically deforming channel walls to physically force fluid motion is by nature a robust technique, since it is insensitive to almost all physicochemical properties of the solution-in marked contrast with most other strategies. Furthermore, local fluid control is attained in straightforward fashion using defined membrane deformations.

These techniques require a material that is soft enough to be deformed using reasonable applied pressures, and the material of choice is typically cross-linked polymeric PDMS. Early fabrication techniques using PDMS (Wilbur et al., 1994; Kim et al., 1995) and PDMSbased microfluidic applications (Delamarche et al., 1997; Effenhauser et al., 1997) have led to an explosion of interest in soft lithography (Xia and Whitesides, 1998; McDonald et al., 2000; Quake and Scherer, 2000; Unger et al., 2000; Whitesides and Stroock, 2001; Whitesides et al., 2001; Ng et al., 2002; Sia and Whitesides, 2003). Advantages include a tunable elastic modulus, a gas permeability that allows dead-end channels to be easily filled and allows an oxygen supply enabling cell cultures (Walker $e t$ al., 2002; Leclerc et al., 2003), and a remarkable ease, speed, and low fabrication cost compared to siliconbased devices (Quake and Scherer, 2000; Whitesides and Stroock, 2001).

Figure 45(a) shows a simple mechanical valve fabricated using multilayer soft lithography, in which a fluid channel is separated from a control channel by a thin membrane. When the control line is pressurized [Fig. 45(b)], the membrane is pushed up to close the fluid channel (Unger et al., 2000; Ismagilov et al., 2001; Studer et al., 2004). Soft passive valves are shown in Figs. 45(c) and $45(\mathrm{~d})$, which open when the fluid behind the valve is
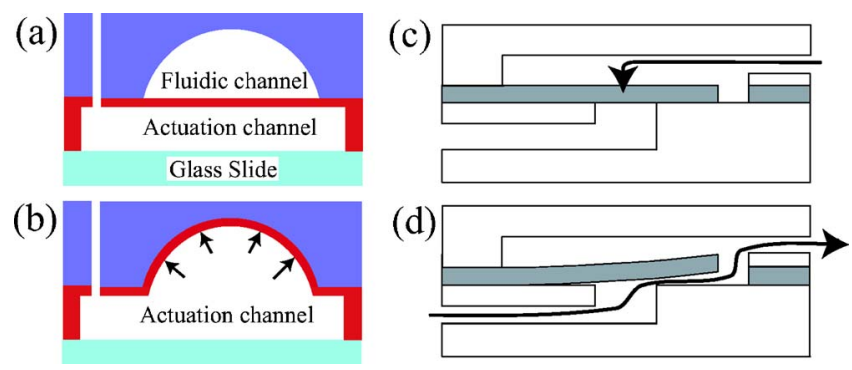

FIG. 45. (Color in online edition) Flexible fluidic valves. (a) An actively controlled valve results when a soft (PDMS) membrane separates a fluidic channel from a gas-filled actuation channel. (b) Pressurizing the gas in the actuation channel pushes the membrane upwards to seal off the fluid channel. A peristaltic pump can be made when three such valves are placed in a row and driven cyclically (Fig. 46). Adapted with permission from Studer et al., 2004. (C2004, AIP. (c,d) A flexible flap that is more easily bent in the forward direction than in reverse forms a passive valve that allows fluid flow in one direction only. Reprinted from Jeon et al., 2002, with kind permission of Springer Science and Business Media.

pressurized, but close when the fluid in front is pressurized (Beebe et al., 2000; Jeon et al., 2002).

Three or more active valves arranged along a channel (Fig. 46) pump fluid peristaltically when the valves are opened and closed cyclically (Unger et al., 2000; Chou et al. 2001). A variety of devices have been designed using such valves and pumps (Hong and Quake, 2003), such as the rotary mixer of Fig. 12 (Chou et al., 2001), the protein crystallization chips of Fig. 32 (Hansen et al., 2002), and protein solubility chip (Hansen et al., 2004), flow injection devices (Leach et al., 2003), and cell analyzers and sorters (Fu et al., 2002; Wheeler et al., 2003). Borrowing from microelectronic large-scale integration strategies, $N_{c}$ fluid chambers can be addressed with a number $N_{l}$ of control lines that increases only logarithmically $\left(N_{l} \sim \ln N_{c}\right)$ (Thorsen et al., 2002). Using this and other ideas for integration, a device has been fabricated that performs 400 PCR reactions using only 41 pipetting steps (as compared to 1200 steps for conventional processes) (Liu, Hansen, and Quake, 2003). This represents significant progress towards achieving the promise of microfluidic "labs on a chip," as the device performs many automated experiments simultaneously while consuming little reagent.

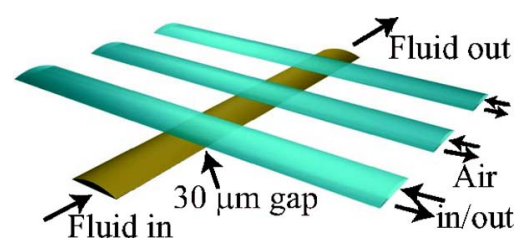

FIG. 46. (Color in online edition) Three active valves (Fig. 45) placed in a row and driven cyclically form a versatile peristaltic pump whose operation is insensitive to almost all physicochemical properties of the solution. Reprinted with permission from Unger et al., 2000. (C2000, AAAS. 
The above examples highlight the deliberate, driven deformation of soft channel elements to manipulate fluids. Because it is soft, PDMS deforms under applied pressures, whether or not such deformations are intended. This may be particularly relevant in light of the high pressures required to drive fluid flows through small channels. The influence of channel deformations on diffusion and dispersion has been examined (Holden et al., 2003), and soft diffraction gratings on channel walls have been used as local pressure sensors (Hosokawa et al., 2002).

More generally, the coupling between local fluid stress and elastic deformation of channel walls has been little studied in the microfluidic context. Compliance causes walls to expand and store extra fluid under applied pressure. Thus compliant walls act like fluidic capacitors, by analogy with electronic circuits. Compliant channel systems thus intrinsically exhibit distributed capacitance and resistance, leading to $R C$ times and low-pass filtering. A low-frequency oscillatory applied pressure deforms the walls to a steady state (fully charging the capacitor) and drives a fluid current through the channel. High-frequency pressures, on the other hand, drive fluid into and out of the space created by deforming channel walls, and drive little appreciable flow.

Fluid-structure interactions have long been studied in the context of biofluid dynamics, as many organisms transport fluid through compliant or collapsible tubes (Grotberg and Jensen, 2004). Although much of this work concerns higher-Re flows, the coupling between the fluid flow and the shape of its surrounding vessel reveals a host of instabilities and nonlinearities that might be exploited for microfluidic flows. For example, a nonlinear fluidic limiter has been designed with compliant elastic walls (King and Toner, 2003).

\section{Intelligent walls made of responsive hydrogel}

The soft microfluidic elements discussed above deform in response to applied pressures. Interesting and useful phenomena arise when channel walls and solid structures contain materials that are themselves active. Intelligent hydrogels have been developed that swell or collapse in response to a wide range of stimuli, e.g., temperature, solvent composition, $p \mathrm{H}$, salinity, electric fields, specific antigens, and light, to list a few (Tanaka, 1978; Tanaka et al., 1980, 1982; Suzuki and Tanaka, 1990; Miyata et al., 1999). An overview of intelligent gels is given by Osada and Rossmurphy (1993), and discussions of their application in the microfluidic context are given by Jager et al. (2000), Moorthy and Beebe (2003), and Roy and Gupta (2003).

Hydrogels can be understood in terms of a polymer gel whose solvent quality changes in response to an environmental change, causing dramatic swelling or deswelling. A more rigorous understanding follows from Flory-Huggins theory, which accounts for entropy and enthalpy of mixing, rubber elasticity, chain-chain interactions, and hydrogen ion dissociation (Flory, 1953; Tanaka, 1978; Tanaka et al., 1982). The dynamics of the
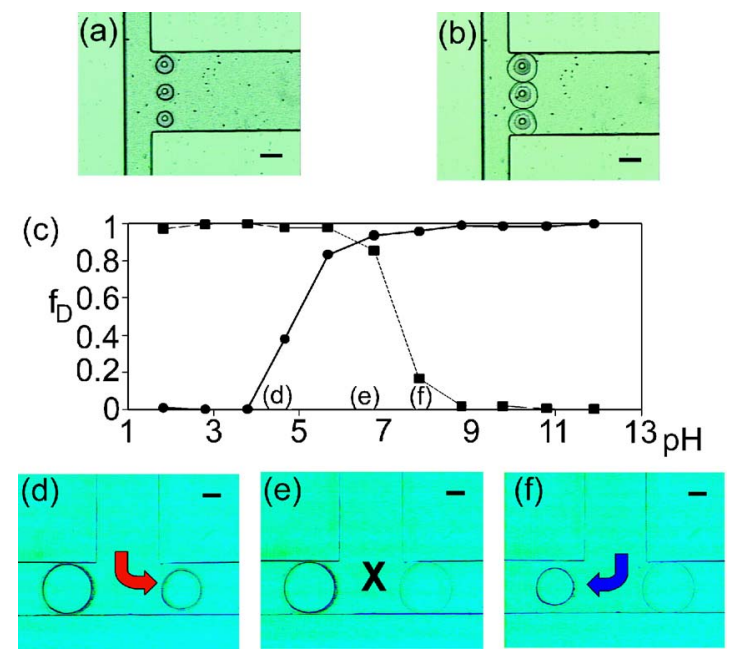

FIG. 47. (Color in online edition) Smart hydrogel-based flow control structures. (a),(b) A $p \mathrm{H}$-responsive valve is formed when $p \mathrm{H}$-sensitive hydrogel posts span a microchannel. (a) The valve open at one $p \mathrm{H}$, and (b) the valve closed at another $p \mathrm{H}$. (c)-(f) A fluid $p \mathrm{H}$ sorter. (c) Two hydrogels are used, one that expands at low $p \mathrm{H}$ and contracts at high $p \mathrm{H}$, and one that does the opposite. (d) At $p \mathrm{H} \sim 4-5$, the left post swells to block the channel, whereas the right shrinks to allow fluid to pass. (e) At intermediate $(\sim 7) \mathrm{pH}$, both posts block the channels, and (f) at high $p \mathrm{H}(\sim 8)$, fluid flows is blocked to the right but flows to the left. Adapted and reprinted with permission from Beebe et al., 2000. (C2000, NPG.

swelling process depend on the collective diffusion of solution into the gel, and thus vary with the square of gel dimension (Matsuo and Tanaka, 1988; De et al., 2002). While slow for macroscopic gels, the smaller dimensions inherent in microfluidic applications yield faster responses, that can be even further diminished without loss of functionality by coating microfabricated solid structures with hydrogel (Beebe et al., 2000).

An intriguing and powerful aspect of such smart valves is that they allow self-regulating fluidic systems. Figure 47 shows two flow regulation devices: the first contains hydrogel-coated posts that swell to block a channel when $p \mathrm{H}$ values are too extreme, and the second sorts fluid by $p \mathrm{H}$ into two channels. One channel contains a hydrogel post that swells at high $p \mathrm{H}$ to allow only low- $p \mathrm{H}$ fluid, and vice versa (Beebe et al., 2000). Furthermore, a self-regulating system has been designed that automatically maintains a constant $p \mathrm{H}$ (Beebe et al., 2000; Eddington et al., 2001; Lee et al., 2003). While these examples all involve $p \mathrm{H}$-sensitive hydrogels, similar devices could be designed that are responsive to other stimuli. Biology uses similar flow control techniques; for example, plant xylem (hollow tubes used to transport water from the roots to the leaves) employ hydrogels to alter hydrodynamic resistance based on the water's ionic strength (Zwieniecki et al., 2001).

Microfluidic valves have been fabricated from thermoresponsive hydrogels (Peters et al., 1997; Harmon et al., 2003; Luo et al., 2003; Richter et al., 2003; Selvaganapathy et al., 2003; Yu et al., 2003), and are typically 
driven using embedded resistive microheaters. Valves have also been made from $p \mathrm{H}$-sensitive hydrogels. (Beebe et al., 2000; Eddington et al., 2001; Yu et al., 2001; Liu, Yu, and Beebe, 2002), and one-shot pumps and valves have been demonstrated using expandable microspheres (Griss et al., 2002). Furthermore, hydrogels have been used as reactors and detectors (Moorthy and Beebe, 2002; Zhan et al., 2002).

We close this section with an interesting phenomenon that has yet to be utilized microfluidically, but which opens intriguing possibilities. Chemical networks whose reactions oscillate spontaneously, known as chemical oscillators, can be systematically designed (Rabai et al., 1990), and key elements of these networks can be incorporated into the hydrogel polymer itself. The result is a structure whose volume oscillates periodically (Yoshida et al., 1996, 2003; Crook et al., 2002; Sakai and Yoshida, 2004). Such self-oscillating structures might find interesting and useful applications in microfluidic devices.

\section{ACKNOWLEDGMENTS}

We are grateful to Armand Ajdari, W. French Anderson, Martin Bazant, John Brady, Gary Leal, Gareth McKinley, and Howard Stone for critical readings of the manuscript, and their many detailed comments and suggestions. Helpful conversations with Jian Gong, Carl Hansen, Eric Lauga, and Aaron Wheeler are gratefully acknowledged. T.M.S. gratefully acknowledges support from a Lee A. Dubridge Prize Postdoctoral Fellowship at Caltech and an NSF Mathematical Sciences Postdoctoral Fellowship.

\section{LIST OF SYMBOLS}

$D \quad$ diffusivity

$D_{z} \quad$ Taylor dispersivity

E electric-field strength

$L_{0} \quad$ generic length scale

$R \quad$ curvature scale

$R_{p}, R_{0} \quad$ polymer size

$T \quad$ temperature

$U_{0} \quad$ velocity scale

$\Delta U \quad$ interspecies velocity difference

$V \quad$ potential difference

$\Delta W \quad$ bandwidth

$Z \quad$ downstream distance scale

$\Delta Z \quad$ band separation

a molecular/particulate radius

$b \quad$ freely jointed chain step length

$c \quad$ capacitance per unit area

$\Delta c \quad$ (dense) solute concentration field

$\dot{e} \quad$ extension rate

$e \quad$ electron charge

f, $f \quad$ body force density on fluid

$f_{v} \quad$ viscous force density

$f_{i} \quad$ inertial force density

$g \quad$ gravitational acceleration

$h \quad$ channel height

$\begin{array}{ll}k_{H} & \text { polymer spring constant } \\ k_{B} & \text { Boltzmann constant } \\ l & \text { channel length } \\ n & \text { number density } \\ p & \text { pressure } \\ r & \text { radial coordinate } \\ \mathbf{u} & \text { fluid velocity field } \\ u_{s} & \text { slip velocity } \\ w & \text { channel width } \\ x & \text { coordinate across channel width } \\ y & \text { coordinate spanning channel height } \\ z & \text { downstream coordinate } \\ \alpha & \text { attenuation length } \\ \alpha_{\rho} & \text { compressibility } \\ \alpha_{T} & \text { coefficient of thermal expansion } \\ \alpha_{c} & \text { density coefficient for species } c \\ \beta & \text { slip length } \\ \delta & \text { oscillatory boundary layer thickness } \\ \epsilon_{w} & \text { dielectric constant of water } \\ \zeta & \text { zeta potential } \\ \eta & \text { shear viscosity } \\ \dot{\gamma} & \text { fluid shear rate } \\ \gamma & \text { liquid-liquid surface tension } \\ \gamma_{s l} & \text { solid-liquid surface tension } \\ \gamma_{s g} & \text { solid-gas surface tension } \\ \kappa_{H} & \text { thermal diffusivity } \\ \lambda_{D} & \text { Debye screening length } \\ \lambda_{B} & \text { Bjerrum length } \\ \mu_{E} & \text { electrophoretic mobility } \\ \nu=\eta / \rho & \text { kinematic viscosity } \\ \rho & \text { fluid density } \\ \rho_{e} & \text { charge density } \\ \rho_{c} & \text { surfactant concentration } \\ \overrightarrow{\boldsymbol{\sigma}}, \sigma & \text { stress tensor } \\ \tau_{D} & \text { diffusion time } \\ \tau_{\text {flow }} & \text { flow time scale } \\ \tau_{i} & \text { inertial time scale } \\ \tau_{p} & \text { polymer relaxation time } \\ \xi & \text { hydrodynamic resistance of bead } \\ \omega & \text { oscillation frequency } \\ & \end{array}$

\section{REFERENCES}

Abbott, S., J. Ralston, G. Reynolds, and R. Hayes, 1999, Langmuir 15, 8923.

Absolom, D. R., W. Zingg, and A. W. Neumann, 1987, J. Biomed. Mater. Res. 21, 161.

Acharya, B. R., T. Krupenkin, S. Ramachandran, Z. Wang, C. C. Huang, and J. A. Rogers, 2003, Appl. Phys. Lett. 83, 4912. Ajdari, A., 1995, Phys. Rev. Lett. 75, 755.

Ajdari, A., 1996, Phys. Rev. E 53, 4996.

Ajdari, A., 2000, Phys. Rev. E 61, R45.

Ajdari, A., 2002, Phys. Rev. E 65, 016301.

Ajdari, A., and J. Prost, 1992, C. R. Acad. Sci., Ser. IIb: Mec., Phys., Chim., Astron. 315, 1635.

Al-Mubaiyedh, U. A., R. Sureshkumar, and B. Khomami, 1999, Phys. Fluids 11, 3217.

Anderson, J. L., 1985, J. Colloid Interface Sci. 105, 45.

Anderson, J. L., 1989, Annu. Rev. Fluid Mech. 21, 61. 
Anderson, J. L., and W. K. Idol, 1985, Chem. Eng. Commun. 38, 93.

Andrienko, D., B. Dunweg, and O. I. Vinogradova, 2003, J. Chem. Phys. 119, 13106.

Angilella, J. R., and J. P. Brancher, 2003, Phys. Fluids 15, 261. Anna, S. L., N. Bontoux, and H. A. Stone, 2003, Appl. Phys. Lett. 82, 364.

Aref, H., 1984, J. Fluid Mech. 143, 1.

Aref, H., 2002, Phys. Fluids 14, 1315.

Aref, H., and S. Balachandar, 1986, Phys. Fluids 29, 3515.

Aris, R., 1956, Proc. R. Soc. London, Ser. A 235, 67.

Aris, R., 1959, Proc. R. Soc. London, Ser. A 252, 538.

Arkilic, E. B., K. S. Breuer, and M. A. Schmidt, 2001, J. Fluid Mech. 437, 29.

Ashton, R., C. Padala, and R. S. Kane, 2003, Curr. Opin. Biotechnol. 14, 497.

Astumian, R. D., and M. Bier, 1994, Phys. Rev. Lett. 72, 1766. Attard, P., 2003, Adv. Colloid Interface Sci. 104, 75.

Auroux, P. A., D. Iossifidis, D. R. Reyes, and A. Manz, 2002, Anal. Chem. 74, 2637.

Auroux, P.-A., Y. Koc, A. deMello, A. Manz, and P. J. R. Day, 2004, Lab Chip 4, 534.

Austin, R. H., N. Darnton, R. Huang, J. Sturm, O. Bakajin, and T. Duke, 2002, Appl. Phys. A: Mater. Sci. Process. 75, 279.

Austin, R. H., and W. D. Volkmuth, 1993, Analusis 21, 235.

Babcock, H. P., D. E. Smith, J. S. Hur, E. S. G. Shaqfeh, and S. Chu, 2000, Phys. Rev. Lett. 85, 2018.

Bain, C. D., 2001, ChemPhysChem 2, 580.

Bain, C. D., G. D. Burnetthall, and R. R. Montgomerie, 1994, Nature (London) 372, 414.

Bajer, K., and H. K. Moffatt, 1990, J. Fluid Mech. 212, 337.

Bakajin, O., T. A. J. Duke, J. Tegenfeldt, C. F. Chou, S. S. Chan, R. H. Austin, and E. C. Cox, 2001, Anal. Chem. 73, 6053.

Bakajin, O. B., T. A. J. Duke, C. F. Chou, S. S. Chan, R. H. Austin, and E. C. Cox, 1998, Phys. Rev. Lett. 80, 2737.

Barbier, V., and J. L. Viovy, 2003, Curr. Opin. Biotechnol. 14, 51.

Baroud, C. N., F. Okkels, L. Menetrier, and P. Tabeling, 2003, Phys. Rev. E 67, 060104.

Barrat, J. L., and L. Bocquet, 1999a, Faraday Discuss. 112, 119. Barrat, J. L., and L. Bocquet, 1999b, Phys. Rev. Lett. 82, 4671. Bassous, E., H. H. Taub, and L. Kuhn, 1977, Appl. Phys. Lett. 31, 135.

Batchelor, G. K., 1977, "Developments in microhydrodynamics," Theoretical and Applied Mechanics, edited by W. T. Koiter (North-Holland, Amsterdam).

Batchelor, G. K., 2001, An Introduction to Fluid Mechanics (Cambridge University Press, Cambridge, England).

Baudry, J., E. Charlaix, A. Tonck, and D. Mazuyer, 2001, Langmuir 17, 5232.

Baumert, B. M., and S. J. Muller, 1995, Rheol. Acta 34, 147.

Baygents, J. C., and F. Baldessari, 1998, Phys. Fluids 10, 301.

Bazant, M. Z., and T. M. Squires, 2004, Phys. Rev. Lett. 92, 066101.

Bazant, M. Z., K. Thornton, and A. Ajdari, 2004, Phys. Rev. E 70, 021506.

Beebe, D. J., G. A. Mensing, and G. M. Walker, 2002, Annu. Rev. Biomed. Eng. 4, 261.

Beebe, D. J., J. S. Moore, J. M. Bauer, Q. Yu, R. H. Liu, C. Devadoss, and B. H. Jo, 2000, Nature (London) 404, 588.

Belaubre, P., M. Guirardel, G. Garcia, J. B. Pourciel, V. Leb- erre, A. Dagkessamanskaia, E. Trevisiol, J. M. Francois, and C. Bergaud, 2003, Appl. Phys. Lett. 82, 3122.

Belgrader, P., W. Benett, D. Hadley, J. Richards, P. Stratton, R. Mariella, and F. Milanovich, 1999, Science 284, 449.

Ben, Y., and H. C. Chang, 2002, J. Fluid Mech. 461, 229.

Beni, G., and S. Hackwood, 1981, Appl. Phys. Lett. 38, 207.

Beni, G., S. Hackwood, and J. L. Jackel, 1982, Appl. Phys. Lett. 40, 912.

Beni, G., and M. A. Tenan, 1981, J. Appl. Phys. 52, 6011.

Bennett, D. E., B. S. Gallardo, and N. L. Abbott, 1996, J. Am. Chem. Soc. 118, 6499.

Berge, B., and J. Peseux, 2000, Eur. Phys. J. E 3, 159.

Bico, J., and D. Quere, 2000, Europhys. Lett. 51, 546.

Bico, J., and D. Quere, 2002, J. Fluid Mech. 467, 101.

Bienia, M., C. Quilliet, and M. Vallade, 2003, Langmuir 19, 9328.

Bier, M., and R. D. Astumian, 1996, Phys. Rev. Lett. 76, 4277.

Bird, R. B., R. C. Armstrong, and O. Hassager, 1987, Dynamics of Polymeric Liquids, Fluid Mechanics No. 1 (Wiley, New York).

Bird, R. B., O. Hassager, R. C. Armstrong, and C. F. Curtiss, 1987, Dynamics of Polymeric Liquids, Kinetic Theory No. 2 (Wiley, New York).

Blake, T. D., A. Clarke, and E. H. Stattersfield, 2000, Langmuir 16, 2928.

Boehnke, U. C., T. Remmler, H. Motschmann, S. Wurlitzer, J. Hauwede, and T. M. Fischer, 1999, J. Colloid Interface Sci. 211, 243.

Boger, D. V., 1977, J. Non-Newtonian Fluid Mech. 3, 87.

Boger, D. V., 1987, Annu. Rev. Fluid Mech. 19, 157.

Boger, D. V., and Y. L. Yeow, 1992, Exp. Therm. Fluid Sci. 5, 633.

Bonaccurso, E., H. J. Butt, and V. S. J. Craig, 2003, Phys. Rev. Lett. 90, 144501.

Bonaccurso, E., M. Kappl, and H. J. Butt, 2002, Phys. Rev. Lett. 88, 076103.

Bottausci, F., I. Mezic, C. D. Meinhart, and C. Cardonne, 2004, Philos. Trans. R. Soc. London, Ser. A 362, 1001.

Bousse, L., C. Cohen, T. Nikiforov, A. Chow, A. R. Kopf-Sill, R. Dubrow, and J. W. Parce, 2000, Annu. Rev. Biophys. Biomol. Struct. 29, 155.

Braun, D., N. L. Goddard, and A. Libchaber, 2003, Phys. Rev. Lett. 91, 158103.

Braun, D., and A. Libchaber, 2002, Phys. Rev. Lett. 89, 188103. Brenner, H., and D. A. Edwards, 1993, Macrotransport Processes (Butterworth-Heinemann, Boston).

Bretherton, F. P., 1961, J. Fluid Mech. 10, 166.

Bringer, M. R., C. J. Gerdts, H. Song, J. D. Tice, and R. F. Ismagilov, 2004, Philos. Trans. R. Soc. London, Ser. A 362, 1087.

Brochard, F., 1989, Langmuir 5, 432.

Brochard, F., and P. G. de Gennes, 1977, J. Chem. Phys. 67, 52. Brochard-Wyart, F., 1995, Europhys. Lett. 30, 387.

Brody, J. P., and P. Yager, 1997, Sens. Actuators, A 58, 13.

Brody, J. P., P. Yager, R. E. Goldstein, and R. H. Austin, 1996, Biophys. J. 71, 3430.

Brown, A. B. D., C. G. Smith, and A. R. Rennie, 2001, Phys. Rev. E 63, 016305.

Bruin, G. J. M., 2000, Electrophoresis 21, 3931.

Bryden, M. D., and H. Brenner, 1999, J. Fluid Mech. 379, 319.

Brzoska, J. B., F. Brochard-Wyart, and F. Rondelez, 1993, Langmuir 9, 2220.

Buch, J. S., P. C. Wang, D. L. DeVoe, and C. S. Lee, 2001, 
Electrophoresis 22, 3902.

Burns, M. A., B. N. Johnson, S. N. Brahmasandra, K. Handique, J. R. Webster, M. Krishnan, T. S. Sammarco, P. M. Man, D. Jones, D. Heldsinger, C. H. Mastrangelo, and D. T. Burke, 1998, Science 282, 484.

Burns, M. A., C. H. Mastrangelo, T. S. Sammarco, F. P. Man, J. R. Webster, B. N. Johnson, B. Foerster, D. Jones, Y. Fields, A. R. Kaiser, and D. T. Burke, 1996, Proc. Natl. Acad. Sci. U.S.A. 93, 5556.

Bustamante, C., J. F. Marko, E. D. Siggia, and S. Smith, 1994, Science 265, 1599.

Byars, J. A., A. Oztekin, R. A. Brown, and G. H. McKinley, 1994, J. Fluid Mech. 271, 173.

Cabodi, M., Y. F. Chen, S. W. P. Turner, H. G. Craighead, and R. H. Austin, 2002, Electrophoresis 23, 3496.

Campbell, C. J., and B. A. Grzybowski, 2004, Philos. Trans. R. Soc. London, Ser. A 362, 1069.

Castelain, C., D. Berger, P. Legentilhomme, A. Mokrani, and H. Peerhossaini, 2000, Int. J. Heat Mass Transfer 43, 3687.

Chabbra, R. P., and D. De Kee, 1992, Transport Processes in Bubbles, Drops and Particles (Hemisphere, New York).

Chandrasekhar, S., 1981, Hydrodynamic and Hydromagnetic Stability (Dover, New York).

Chaudhury, M. K., and G. M. Whitesides, 1992, Science 256, 1539.

Cheikh, C., and G. Koper, 2003, Phys. Rev. Lett. 91, 156102.

Chen, C.-H., H. Lin, S. K. Lele, and J. G. Santiago, 2005, J. Fluid Mech. 524, 263.

Chen, H., and J. C. Meiners, 2004, Appl. Phys. Lett. 84, 2193.

Cheng, J., M. A. Shoffner, G. E. Hvichia, L. J. Kricka, and P. Wilding, 1996, Nucleic Acids Res. 24, 380.

Cheng, J. T., and N. Giordano, 2002, Phys. Rev. E 65, 031206. Chiou, J., P. Matsudaira, A. Sonin, and D. Ehrlich, 2001, Anal. Chem. 73, 2018.

Chiou, P. Y., H. Moon, H. Toshiyoshi, C. J. Kim, and M. C. Wu, 2003, Sens. Actuators, A 104, 222.

Cho, B. S., T. G. Schuster, X. Zhu, D. Chang, G. D. Smith, and S. Takayama, 2003, Anal. Chem. 75, 1671.

Cho, S. K., H. J. Moon, and C. J. Kim, 2003, J. Microelectromech. Syst. 12, 70.

Choban, E. R., L. J. Markoski, A. Wieckowski, and P. J. A. Kenis, 2004, J. Power Sources 128, 54.

Choi, C. H., K. J. A. Westin, and K. S. Breuer, 2003, Phys. Fluids 15, 2897.

Chou, C. F., R. H. Austin, O. Bakajin, J. O. Tegenfeldt, J. A. Castelino, S. S. Chan, E. C. Cox, H. Craighead, N. Darnton, T. Duke, J. Y. Han, and S. Turner, 2000, Electrophoresis 21, 81.

Chou, C. F., O. Bakajin, S. W. P. Turner, T. A. J. Duke, S. S. Chan, E. C. Cox, H. G. Craighead, and R. H. Austin, 1999, Proc. Natl. Acad. Sci. U.S.A. 96, 13762.

Chou, H. P., M. A. Unger, and S. Quake, 2001, Biomed. Microdevices 3, 323.

Churaev, N. V., V. D. Sobolev, and A. N. Somov, 1984, J. Colloid Interface Sci. 97, 574.

Cieplak, M., J. Koplik, and J. R. Banavar, 2001, Phys. Rev. Lett. 86, 803 .

Cochrane, T., K. Walters, and M. F. Webster, 1981, Philos. Trans. R. Soc. London, Ser. A 301, 163.

Colgate, E., and H. Matsumoto, 1990, J. Vac. Sci. Technol. A 8, 3625 .

Cottin-Bizonne, C., J. L. Barrat, L. Bocquet, and E. Charlaix, 2003, Nat. Mater. 2, 237.
Cottin-Bizonne, C., S. Jurine, J. Baudry, J. Crassous, F. Restagno, and E. Charlaix, 2002, Eur. Phys. J. E 9, 47.

Craig, V. S. J., C. Neto, and D. R. M. Williams, 2001, Phys. Rev. Lett. 87, 054504.

Crook, C. J., A. Smith, R. A. L. Jones, and A. J. Ryan, 2002, Phys. Chem. Chem. Phys. 4, 1367.

Crut, A., D. Lasne, J. F. Allemand, M. Dahan, and P. Desbiolles, 2003, Phys. Rev. E 67, 051910.

Culbertson, C. T., S. C. Jacobson, and J. M. Ramsey, 2000, Anal. Chem. 72, 5814.

Daniel, S., M. K. Chaudhury, and J. C. Chen, 2001, Science 291, 633.

Darhuber, A. A., J. Z. Chen, J. M. Davis, and S. M. Troian, 2004, Philos. Trans. R. Soc. London, Ser. A 362, 1037.

Darhuber, A. A., J. M. Davis, S. M. Troian, and W. W. Reisner, 2003, Phys. Fluids 15, 1295.

Darhuber, A. A., S. M. Troian, S. M. Miller, and S. Wagner, 2000, J. Appl. Phys. 87, 7768.

Darhuber, A. A., S. M. Troian, and W. W. Reisner, 2001, Phys. Rev. E 64, 031603.

Darhuber, A. A., S. M. Troian, and S. Wagner, 2002, J. Appl. Phys. 91, 5686.

Darhuber, A. A., J. P. Valentino, J. M. Davis, S. M. Troian, and S. Wagner, 2003, Appl. Phys. Lett. 82, 657.

Davis, S. H., 1987, Annu. Rev. Fluid Mech. 19, 403.

De, S. K., N. R. Aluru, B. Johnson, W. C. Crone, D. J. Beebe, and J. Moore, 2002, J. Microelectromech. Syst. 11, 544.

Dean, W. R., 1927, Philos. Mag. 4, 208.

Dean, W. R., 1928, Philos. Mag. 5, 673.

Deen, W. M., 1998, Analysis of Transport Phenomena (Oxford University Press, New York).

de Gennes, P. G., 1974, J. Chem. Phys. 60, 5030.

de Gennes, P. G., 1985, Rev. Mod. Phys. 57, 827.

de Gennes, P. G., 1997, Science 276, 1999.

de Gennes, P. G., 1998, Physica A 249, 196.

de Gennes, P. G., 2002, Langmuir 18, 3413.

de Gennes, P. G., F. Brochard-Wyart, and D. Quere, 2004, Capillary and Wetting Phenomena: Drops, Bubbles, Pearls, Waves (Springer-Verlag, New York).

Delamarche, E., A. Bernard, H. Schmid, B. Michel, and H. Biebuyck, 1997, Science 276, 779.

DeLucas, L. J., K. M. Moore, M. M. Long, R. Rouleau, T. Bray, W. Crysel, and L. Weise, 2002, J. Cryst. Growth 237, 1646.

DeLucas, L. J., et al., 1989, Science 246, 651.

Dolnik, V., 1997, Electrophoresis 18, 2353.

Dolnik, V., 1999, Electrophoresis 20, 3106.

Dolnik, V., and K. M. Hutterer, 2001, Electrophoresis 22, 4163.

Dolnik, V., S. R. Liu, and S. Jovanovich, 2000, Electrophoresis 21, 41.

Dorfman, K. D., and J. L. Viovy, 2004, Phys. Rev. E 69, 011901.

Dos Santos, D. F., and T. Ondarçuhu, 1995, Phys. Rev. Lett. 75, 2972.

Doyle, P. S., J. Bibette, A. Bancaud, and J. L. Viovy, 2002, Science 295, 2237.

Doyle, P. S., B. Ladoux, and J. L. Viovy, 2000, Phys. Rev. Lett. 84, 4769.

Dreyfus, R., P. Tabeling, and H. Willaime, 2003, Phys. Rev. Lett. 90, 144505.

Duke, T., G. Monnelly, R. H. Austin, and E. C. Cox, 1997, Electrophoresis 18, 17.

Duke, T. A. J., and R. H. Austin, 1998, Phys. Rev. Lett. 80, 
1552.

Duke, T. A. J., R. H. Austin, E. C. Cox, and S. S. Chan, 1996, Electrophoresis 17, 1075.

Dukhin, A. S., 1986, Colloid J. USSR 48, 376.

Dukhin, A. S., and V. A. Murtsovkin, 1986, Colloid J. USSR 48, 203.

Dussan, E. B., 1979, Annu. Rev. Fluid Mech. 11, 371.

Dutta, D., and D. T. Leighton, 2001, Anal. Chem. 73, 504.

Dutta, D., and D. T. Leighton, 2002, Anal. Chem. 74, 1007.

Eaton, W. A., V. Munoz, S. J. Hagen, G. S. Jas, L. J. Lapidus, E. R. Henry, and J. Hofrichter, 2000, Annu. Rev. Biophys. Biomol. Struct. 29, 327.

Eckart, C., 1948, J. Mar. Res. 7, 265.

Eddington, D. T., R. H. Liu, J. S. Moore, and D. J. Beebe, 2001, Lab Chip 1, 96.

Effenhauser, C. S., G. J. M. Bruin, A. Paulus, and M. Ehrat, 1997, Anal. Chem. 69, 3451.

Eggers, J., and H. A. Stone, 2004, J. Fluid Mech. 505, 309.

El Moctar, A. O., N. Aubry, and J. Batton, 2003, Lab Chip 3, 273.

Elder, S. A., 1959, J. Acoust. Soc. Am. 31, 54.

Erickson, D., and D. Q. Li, 2002a, Langmuir 18, 1883.

Erickson, D., and D. Q. Li, 2002b, Langmuir 18, 8949.

Ertas, D., 1998, Phys. Rev. Lett. 80, 1548.

Fair, M. C., 1990, Int. J. Multiphase Flow 16, 1131.

Fair, M. C., and J. L. Anderson, 1989, J. Colloid Interface Sci. 127, 388

Fair, M. C., and J. L. Anderson, 1990, Int. J. Multiphase Flow 16, 663.

Fair, M. C., and J. L. Anderson, 1992, Langmuir 8, 2850.

Ferrigno, R., A. D. Stroock, T. D. Clark, M. Mayer, and G. M. Whitesides, 2002, J. Am. Chem. Soc. 124, 12930.

Feynman, R. P., R. B. Leighton, and M. L. Sands, 1989, The Feynman Lectures on Physics, Vol. 2 (Addison-Wesley, Reading, MA).

Fiechtner, G. J., and E. B. Cummings, 2003, Anal. Chem. 75, 4747.

Fiechtner, G. J., and E. B. Cummings, 2004, J. Chromatogr., A 1027, 245.

Figeys, D., and D. Pinto, 2000, Anal. Chem. 72, 330A.

Figeys, D., and D. Pinto, 2001, Electrophoresis 22, 208.

Flory, P. J., 1953, Principles of Polymer Chemistry (Cornell, Ithaca).

Foster, K., and G. A. Parker, 1970, Fluidics: Components and Circuits (Wiley Interscience, London).

Frampton, K. D., S. E. Martin, and K. Minor, 2003, Appl. Acoust. 64, 681.

Frumkin, A., and V. Levich, 1945, Acta Physicochim. URSS 20, 769.

Fu, A. Y., H. P. Chou, C. Spence, F. H. Arnold, and S. R. Quake, 2002, Anal. Chem. 74, 2451.

Gad-el Hak, M., 1999, J. Fluids Eng. 121, 5.

Gajar, S. A., and M. W. Geis, 1992, J. Electrochem. Soc. 139, 2833.

Gallardo, B. S., and N. L. Abbott, 1997, Langmuir 13, 203.

Gallardo, B. S., V. K. Gupta, F. D. Eagerton, L. I. Jong, V. S. Craig, R. R. Shah, and N. L. Abbott, 1999, Science 283, 57.

Gallardo, B. S., M. J. Hwa, and N. L. Abbott, 1995, Langmuir 11, 4209.

Gallardo, B. S., K. L. Metcalfe, and N. L. Abbott, 1996, Langmuir 12, 4116.

Gamayunov, N. I., G. I. Mantrov, and V. A. Murtsovkin, 1992, Colloid J. 54, 20.
Gamayunov, N. I., V. A. Murtsovkin, and A. S. Dukhin, 1986, Colloid J. USSR 48, 197.

Ganan-Calvo, A. M., 1998, Phys. Rev. Lett. 80, 285.

Ganan-Calvo, A. M., and J. M. Gordillo, 2001, Phys. Rev. Lett. 87, 274501.

Garcia-Ruiz, J. M., M. L. Novella, R. Moreno, and J. A. Gavira, 2001, J. Cryst. Growth 232, 165.

Garmer, N., R. O. Grigoriev, and M. F. Schatz, 2003, Phys. Rev. Lett. 91, 054501.

Garstecki, P., I. Gitlin, W. DiLuzio, G. M. Whitesides, E. Kumacheva, and H. A. Stone, 2004, Appl. Phys. Lett. 85, 2649.

Gascoyne, P., C. Mahidol, M. Ruchirawat, J. Satayavivad, P. Watcharasit, and F. F. Becker, 2002, Lab Chip 2, 70.

Gascoyne, P. R. C., and J. V. Vykoukal, 2004, Proc. IEEE 92, 22.

Gau, H., S. Herminghaus, P. Lenz, and R. Lipowsky, 1999, Science 283, 46.

Gaver, D. P., and J. B. Grotberg, 1986, J. Fluid Mech. 172, 47. Gee, M. L., P. M. McGuiggan, J. N. Israelachvili, and A. M. Homola, 1990, J. Chem. Phys. 93, 1895.

Ghosal, S., 2002a, Anal. Chem. 74, 4198.

Ghosal, S., 2002b, J. Fluid Mech. 459, 103.

Ghosal, S., 2003, J. Fluid Mech. 491, 285.

Ghosal, S., 2004, Electrophoresis 25, 214.

Ghowsi, K., and R. J. Gale, 1991, J. Chromatogr. 559, 95.

Giddings, J. C., 1991, Unified Separation Science (Wiley, New York).

Gitlin, I., A. D. Stroock, G. M. Whitesides, and A. Ajdari, 2003, Appl. Phys. Lett. 83, 1486.

Glasgow, I., and N. Aubry, 2003, Lab Chip 3, 114.

Gleeson, J. P., O. M. Roche, J. West, and A. Gelb, 2004, SIAM J. Appl. Math. 64, 1294.

Gluckstad, J., 2004, Nat. Mater. 3, 9.

Goldstein, S., 1938, Modern Developments in Fluid Dynamics (Clarendon Press, Oxford).

Gong, T. Y., D. T. Wu, and D. W. M. Marr, 2002, Langmuir 18, 10064.

Gong, T. Y., D. T. Wu, and D. W. M. Marr, 2003, Langmuir 19, 5967.

Gonzalez, A., A. Ramos, N. G. Green, A. Castellanos, and H. Morgan, 2000, Phys. Rev. E 61, 4019.

Gordillo, J. M., A. M. Ganan-Calvo, and M. Perez-Saborid, 2001, Phys. Fluids 13, 3839.

Graham, M. D., 1998, J. Fluid Mech. 360, 341.

Graham, M. D., 2003, Phys. Fluids 15, 1702.

Granick, S., Y. X. Zhu, and H. Lee, 2003, Nat. Mater. 2, 221. Grant, M. L., and D. A. Saville, 1991, J. Cryst. Growth 108, 8. Green, N. G., A. Ramos, A. Gonzalez, H. Morgan, and A. Castellanos, 2000, Phys. Rev. E 61, 4011.

Green, N. G., A. Ramos, A. Gonzalez, H. Morgan, and A. Castellanos, 2002, Phys. Rev. E 66, 026305.

Green, N. G., A. Ramos, and H. Morgan, 2000, J. Phys. D 33, 632.

Greenspan, H. P., 1978, J. Fluid Mech. 84, 125.

Griffiths, S. K., and R. H. Nilson, 2000, Anal. Chem. 72, 5473. Griffiths, S. K., and R. H. Nilson, 2002, Anal. Chem. 74, 2960. Griss, P., H. Andersson, and G. Stemme, 2002, Lab Chip 2, 117.

Groisman, A., M. Enzelberger, and S. R. Quake, 2003, Science 300, 955.

Groisman, A., and S. Quake, 2004, Phys. Rev. Lett. 92, 094501. Groisman, A., and V. Steinberg, 2000, Nature (London) 405, 53. 
Groisman, A., and V. Steinberg, 2001, Nature (London) 410 , 905.

Groisman, A., and V. Steinberg, 2004, New J. Phys. 6, 29.

Grotberg, J. B., 1984, J. Fluid Mech. 141, 249.

Grotberg, J. B., and O. E. Jensen, 2004, Annu. Rev. Fluid Mech. 36, 121.

Gueroui, Z., E. Freyssingeas, C. Place, and B. Berge, 2003, Eur. Phys. J. E 11, 105.

Hall, P., 1974, J. Fluid Mech. 64, 209.

Hancock, W. S., S.-L. Wu, and P. Shieh, 2002, Proteomics 2, 352.

Handique, K., and M. A. Burns, 2001, J. Micromech. Microeng. 11, 548.

Handique, K., B. P. Gogoi, D. T. Burke, C. H. Mastrangelo, and M. A. Burns, 1997, Proc. SPIE 3224, 185.

Hansen, C., and S. R. Quake, 2003, Curr. Opin. Struct. Biol. 13, 538 .

Hansen, C., M. O. A. Sommer, and S. R. Quake, 2004, Proc. Natl. Acad. Sci. U.S.A. 101, 14431.

Hansen, C. L., E. Skordalakes, J. M. Berger, and S. R. Quake, 2002, Proc. Natl. Acad. Sci. U.S.A. 99, 16531.

Happel, J., and H. Brenner, 1983, Low Reynolds Number Hydrodynamics (Martinus Nijhoff, The Hague).

Harmon, M. E., M. Tang, and C. W. Frank, 2003, Polymer 44, 4547.

Harrison, D. J., K. Fluri, K. Seiler, Z. H. Fan, C. S. Effenhauser, and A. Manz, 1993, Science 261, 895.

Harrison, D. J., A. Manz, Z. H. Fan, H. Ludi, and H. M. Widmer, 1992, Anal. Chem. 64, 1926.

Hatch, A., A. E. Kamholz, K. R. Hawkins, M. S. Munson, E. A. Schilling, B. H. Weigl, and P. Yager, 2001, Nat. Biotechnol. 19, 461.

Hayes, R. A., and B. J. Feenstra, 2003, Nature (London) 425, 383.

Henry, A. C., E. A. Waddell, R. Shreiner, and L. E. Locascio, 2002, Electrophoresis 23, 791.

Herr, A. E., J. I. Molho, J. G. Santiago, M. G. Mungal, T. W. Kenny, and M. G. Garguilo, 2000, Anal. Chem. 72, 1053.

Hinch, E. J., 1977, Phys. Fluids 20, s22.

Hinch, E. J., 1988, in Disorder and Mixing, edited by E. Guyon, J.-P. Nadal, and Y. Pomeau (Kluwer Academic, Dordrecht), pp. 43-55.

Ho, C.-M., and Y.-C. Tai, 1998, Annu. Rev. Fluid Mech. 30, 579.

Hoburg, J. F., and J. R. Melcher, 1976, J. Fluid Mech. 73, 333.

Hoburg, J. F., and J. R. Melcher, 1977, Phys. Fluids 20, 903.

Holden, M. A., S. Kumar, A. Beskok, and P. S. Cremer, 2003, J. Micromech. Microeng. 13, 412.

Hollingsworth, A. D., and D. A. Saville, 2003, J. Colloid Interface Sci. 257, 65.

Hong, J. W., T. Fujii, M. Seki, T. Yamamoto, and I. Endo, 2001, Electrophoresis 22, 328.

Hong, J. W., and S. R. Quake, 2003, Nat. Biotechnol. 21, 1179.

Hong, J. W., V. Studer, G. Hang, W. F. Anderson, and S. R. Quake, 2004, Nat. Biotechnol. 22, 435.

Horn, R. G., O. I. Vinogradova, M. E. Mackay, and N. PhanThien, 2000, J. Chem. Phys. 112, 6424.

Hosokawa, K., K. Hanada, and R. Maeda, 2002, J. Micromech. Microeng. 12, 1.

Hsieh, J., P. Mach, F. Cattaneo, S. Yang, T. Krupenkine, K. Baldwin, and J. A. Rogers, 2003, IEEE Photonics Technol. Lett. 15, 81.

Huang, L., Y. Zhang, J. H. Liao, L. N. Xu, N. Gu, and H. J.
Shen, 2003, Surf. Rev. Lett. 10, 569.

Huang, L. R., E. C. Cox, R. H. Austin, and J. C. Sturm, 2004, Science 304, 987.

Huang, L. R., P. Silberzan, J. O. Tegenfeldt, E. C. Cox, J. C. Sturm, R. H. Austin, and H. Craighead, 2002, Phys. Rev. Lett. 89, 178301.

Huang, L. R., J. O. Tegenfeldt, J. J. Kraeft, J. C. Sturm, R. H. Austin, and E. C. Cox, 2002, Nat. Biotechnol. 20, 1048.

Humphrey, E. F., and D. H. Tarumoto, 1965, Eds., Fluidics (Fluid Amplifier Associates, Boston).

Hunter, R. J., 2000, Foundations of Colloid Science, 2nd ed. (Oxford University Press, Oxford).

Hur, J. S., E. S. G. Shaqfeh, H. P. Babcock, and S. Chu, 2002, Phys. Rev. E 66, 011915.

Hur, J. S., E. S. G. Shaqfeh, and R. G. Larson, 2000, J. Rheol. 44, 713.

Huth, J. M., H. L. Swinney, W. D. McCormick, A. Kuhn, and F. Argoul, 1995, Phys. Rev. E 51, 3444.

Ichimura, K., S. K. Oh, and M. Nakagawa, 2000, Science 288, 1624.

Im, D. J., and I. S. Kang, 2003, J. Colloid Interface Sci. 266, 127.

Ishida, N., T. Inoue, M. Miyahara, and K. Higashitani, 2000, Langmuir 16, 6377.

Ismagilov, R. F., D. Rosmarin, P. J. A. Kenis, D. T. Chiu, W. Zhang, H. A. Stone, and G. M. Whitesides, 2001, Anal. Chem. 73, 4682.

Ismagilov, R. F., A. D. Stroock, P. J. A. Kenis, G. Whitesides, and H. A. Stone, 2000, Appl. Phys. Lett. 76, 2376.

Israelachvili, J. N., 1986, J. Colloid Interface Sci. 110, 263.

Jackel, J. L., S. Hackwood, and G. Beni, 1982, Appl. Phys. Lett. 40, 4.

Jackel, J. L., S. Hackwood, J. J. Veselka, and G. Beni, 1983, Appl. Opt. 22, 1765.

Jacobson, S. C., and J. M. Ramsey, 1995, Electrophoresis 16, 481.

Jager, E. W. H., E. Smela, and O. Inganas, 2000, Science 290, 1540.

Janocha, B., H. Bauser, C. Oehr, H. Brunner, and W. Gopel, 2000, Langmuir 16, 3349.

Jansons, K. M., 1988, Phys. Fluids 31, 15.

Jendrejack, R. M., E. T. Dimalanta, D. C. Schwartz, M. D. Graham, and J. J. de Pablo, 2003, Phys. Rev. Lett. 91, 038102.

Jendrejack, R. M., D. C. Schwartz, J. J. de Pablo, and M. D. Graham, 2004, J. Chem. Phys. 120, 2513.

Jendrejack, R. M., D. C. Schwartz, M. D. Graham, and J. J. de Pablo, 2003, J. Chem. Phys. 119, 1165.

Jeon, N. L., D. T. Chiu, C. J. Wargo, H. K. Wu, I. S. Choi, J. R. Anderson, and G. M. Whitesides, 2002, Biomed. Microdevices 4, 117.

Johnson, T. J., and L. E. Locascio, 2002, Lab Chip 2, 135.

Johnson, T. J., D. Ross, M. Gaitan, and L. E. Locascio, 2001, Anal. Chem. 73, 3656.

Johnson, T. J., D. Ross, and L. E. Locascio, 2002, Anal. Chem. 74, 45.

Jones, S. W., O. M. Thomas, and H. Aref, 1989, J. Fluid Mech. 209, 335.

Jones, S. W., and W. R. Young, 1994, J. Fluid Mech. 280, 149. Jones, T. B., 2002, Langmuir 18, 4437.

Jones, T. B., J. D. Fowler, Y. S. Chang, and C. J. Kim, 2003, Langmuir 19, 7646.

Jones, T. B., M. Gunji, M. Washizu, and M. J. Feldman, 2001, J. Appl. Phys. 89, 1441. 
Jong, L. I., and N. L. Abbott, 1998, Langmuir 14, 2235.

Joo, Y. L., and E. S. G. Shaqfeh, 1991, Phys. Fluids A 3, 1691. Joo, Y. L., and E. S. G. Shaqfeh, 1992, Phys. Fluids A 4, 524. Joyce, J. W., 1983, Fluidics-Basic Components and Applications, Special Report HDL-SR-83-9, Harry Diamond Laboratories.

Jun, T. K., and C. J. Kim, 1998, J. Appl. Phys. 83, 5658.

Juncker, D., H. Schmid, U. Drechsler, H. Wolf, M. Wolf, B. Michel, N. de Rooij, and E. Delamarche, 2002, Anal. Chem. 74, 6139.

Jung, B., R. Bharadwaj, and J. G. Santiago, 2003, Electrophoresis 24, 3476.

Kamholz, A. E., E. A. Schilling, and P. Yager, 2001, Biophys. J. 80, 1967.

Kamholz, A. E., B. H. Weigl, B. A. Finlayson, and P. Yager, 1999, Anal. Chem. 71, 5340.

Kamholz, A. E., and P. Yager, 2001, Biophys. J. 80, 155.

Kamholz, A. E., and P. Yager, 2002, Sens. Actuators B 82, 117.

Kang, K. H., 2002, Langmuir 18, 10318.

Kataoka, D. E., and S. M. Troian, 1997, J. Colloid Interface Sci. 192, 350.

Kataoka, D. E., and S. M. Troian, 1998, J. Colloid Interface Sci. 203, 335.

Kataoka, D. E., and S. M. Troian, 1999, Nature (London) 402, 794.

Kauffmann, E., N. C. Darnton, R. H. Austin, C. Batt, and K. Gerwert, 2001, Proc. Natl. Acad. Sci. U.S.A. 98, 6646.

Kenis, P. J. A., R. F. Ismagilov, S. Takayama, G. M. Whitesides, S. L. Li, and H. S. White, 2000, Acc. Chem. Res. 33, 841.

Kenis, P. J. A., R. F. Ismagilov, and G. M. Whitesides, 1999, Science 285, 83.

Khandurina, J., T. E. McKnight, S. C. Jacobson, L. C. Waters, R. S. Foote, and J. M. Ramsey, 2000, Anal. Chem. 72, 2995.

Kim, E., Y. N. Xia, and G. M. Whitesides, 1995, Nature (London) 376, 581.

Kim, S., and S. J. Karilla, 1991, Microhydrodynamics: Principles and Selected Applications (Butterworth-Heinemann, Boston).

King, M. R., and M. Toner, 2003, in Proceedings of MuTAS 2003, edited by M. A. Northrup, K. F. Jensen, and D. J. Harrison (Transducer Research Foundation, Squaw Valley), Vol. 1, pp. 49-52.

Knight, J. B., A. Vishwanath, J. P. Brody, and R. H. Austin, 1998, Phys. Rev. Lett. 80, 3863.

Kopp, M. U., A. J. de Mello, and A. Manz, 1998, Science 280, 1046.

Krishnan, M., V. M. Ugaz, and M. A. Burns, 2002, Science 298, 793.

Kroujiline, D., and H. A. Stone, 1999, Physica D 130, 105.

Krupenkin, T., S. Yang, and P. Mach, 2003, Appl. Phys. Lett. 82, 316.

Krupenkin, T. N., J. A. Taylor, T. M. Schneider, and S. Yang, 2004, Langmuir 20, 3824.

Kuhn, W., 1934, Kolloid-Z. 68, 2.

Kumar, K. A., and M. D. Graham, 2000, J. Non-Newtonian Fluid Mech. 89, 337.

Kuo, J. S., P. Spicar-Mihalic, I. Rodriguez, and D. T. Chiu, 2003, Langmuir 19, 250.

Ladavac, K., K. Kasza, and D. G. Grier, 2004, Phys. Rev. E 70, 010901.

Ladoux, B., and P. S. Doyle, 2000, Europhys. Lett. 52, 511.

Lagally, E. T., C. A. Emrich, and R. A. Mathies, 2001, Lab Chip 1, 102.
Lagally, E. T., I. Medintz, and R. A. Mathies, 2001, Anal. Chem. 73, 565.

Lahann, J., S. Mitragotri, T. N. Tran, H. Kaido, J. Sundaram, I. S. Choi, S. Hoffer, G. A. Somorjai, and R. Langer, 2003, Science 299, 371.

Lajeunesse, E., and G. M. Homsy, 2003, Phys. Fluids 15, 308.

Lambelet, P., A. Sayah, M. Pfeffer, C. Philipona, and F. Marquis-Weible, 1998, Appl. Opt. 37, 7289.

Landau, L., and V. Levich, 1942, Acta Physicochim. URSS 17, 42.

Landers, J. P., 2003, Anal. Chem. 75, 2919.

Larson, R. G., 1988, Constitutive Equations for Polymer Melts and Solutions (Butterworths, Boston).

Larson, R. G., 1992, Rheol. Acta 31, 213.

Larson, R. G., 1999, The Structure and Rheology of Complex

Fluids (Oxford University Press, New York).

Larson, R. G., 2000, Nature (London) 405, 27.

Larson, R. G., H. Hu, D. E. Smith, and S. Chu, 1999, J. Rheol. 43, 267.

Larson, R. G., and J. J. Magda, 1989, Macromolecules 22, 3004.

Larson, R. G., S. J. Muller, and E. S. G. Shaqfeh, 1994, J.

Non-Newtonian Fluid Mech. 51, 195.

Larson, R. G., T. T. Perkins, D. E. Smith, and S. Chu, 1997, Phys. Rev. E 55, 1794.

Larson, R. G., E. S. G. Shaqfeh, and S. J. Muller, 1990, J. Fluid Mech. 218, 573.

Laser, D. J., and J. G. Santiago, 2004, J. Micromech. Microeng. 14, R35.

Lastochkin, D., R. H. Zhou, P. Wang, Y. X. Ben, and H. C. Chang, 2004, J. Appl. Phys. 96, 1730.

Lauga, E., and M. P. Brenner, 2004, Phys. Rev. E 70, 026311.

Lauga, E., and H. A. Stone, 2003, J. Fluid Mech. 489, 55.

Leach, A. M., A. R. Wheeler, and R. N. Zare, 2003, Anal. Chem. 75, 967.

Leal, L. G., 1992, Laminar Flow and Convective Transport Processes: Scaling Principles and Asymptotic Analysis (Butterworth-Heinemann, Boston).

Leclerc, E., Y. Sakai, and T. Fujii, 2003, Biomed. Microdevices 5, 109.

Lee, C. P., and A. A. Chernov, 2002, J. Cryst. Growth 240, 531.

Lee, H. J., D. J. Fermin, R. M. Corn, and H. H. Girault, 1999, Electrochem. Commun. 1, 190.

Lee, J., and C. J. Kim, 2000, J. Microelectromech. Syst. 9, 171.

Lee, J., H. Moon, J. Fowler, T. Schoellhammer, and C. J. Kim, 2002, Sens. Actuators, A 95, 259.

Lee, S. H., D. T. Eddington, Y. M. Kim, W. S. Kim, and D. J. Beebe, 2003, J. Microelectromech. Syst. 12, 848.

Lee, S. M., D. J. Im, and I. S. Kang, 2000, Phys. Fluids 12, 1899.

Lee, S. W., D. Y. Kwok, and P. E. Laibinis, 2002, Phys. Rev. E 65, 051602.

Lee, S. W., and P. E. Laibinis, 2000, J. Am. Chem. Soc. 122, 5395.

Lévêque, M. A., 1928, Ann. Mines 13, 201.

Levich, V. G., 1962, Physicochemical Hydrodynamics (Prentice-Hall, Englewood Cliffs, NJ).

Levich, V. G., and V. S. Krylov, 1969, Annu. Rev. Fluid Mech. 1, 293.

Levich, V. G., and A. M. Kuznetsov, 1962, Dokl. Akad. Nauk SSSR 146, 145.

Levitan, J. A., S. Devasenathipathy, V. Studer, T. Thorsen, T. M. Squires, and M. Z. Bazant, 2005, Colloids Surf., A 267, 122.

Lichtenberg, J., N. F. de Rooij, and E. Verpoorte, 2002, Talanta 
56, 233.

Lichtenberg, J., E. Verpoorte, and N. F. de Rooij, 2001, Electrophoresis 22, 258.

Lide, D. R., 1999, Ed., CRC Handbook of Chemistry and Physics, 79th ed. (CRC Press, Boca Raton).

Lighthill, J., 1978, J. Sound Vib. 61, 391.

Lighthill, J., 2001, Waves in Fluids (Cambridge University Press, Cambridge, England).

Lin, H., B. D. Storey, M. H. Oddy, C.-H. Chen, and J. G. Santiago, 2004, Phys. Fluids 16, 1922.

Link, D. R., S. L. Anna, D. A. Weitz, and H. A. Stone, 2004, Phys. Rev. Lett. 92, 054503.

Lion, N., et al., 2003, Electrophoresis 24, 3533.

Lipman, E. A., B. Schuler, O. Bakajin, and W. A. Eaton, 2003, Science 301, 1233.

Lippmann, G., 1875, Ann. Chim. Phys. 5, 494.

Liu, J., M. Enzelberger, and S. Quake, 2002, Electrophoresis 23, 1531.

Liu, J., C. Hansen, and S. R. Quake, 2003, Anal. Chem. 75, 4718.

Liu, R. H., R. Lenigk, R. L. Druyor-Sanchez, J. Yang, and P. Grodzinski, 2003, Anal. Chem. 75, 1911.

Liu, R. H., M. A. Stremler, K. V. Sharp, M. G. Olsen, J. G. Santiago, R. J. Adrian, H. Aref, and D. J. Beebe, 2000, J. Microelectromech. Syst. 9, 190.

Liu, R. H., J. Yang, M. Z. Pindera, M. Mathavale, and P. Grodzinski, 2002, Lab Chip 2, 151.

Liu, R. H., Q. Yu, and D. J. Beebe, 2002, J. Microelectromech. Syst. 11, 45.

Liu, T. W., 1989, J. Chem. Phys. 90, 5826.

Long, D., and A. Ajdari, 1996, Electrophoresis 17, 1161.

Long, D., and A. Ajdari, 1998, Phys. Rev. Lett. 81, 1529.

Long, D., H. A. Stone, and A. Ajdari, 1999, J. Colloid Interface Sci. 212, 338.

Long, D., J. L. Viovy, and A. Ajdari, 1996, Phys. Rev. Lett. 76, 3858.

Longuet-Higgins, M. S., 1998, Proc. R. Soc. London, Ser. A 454, 725.

Ludviksson, V., and E. N. Lightfoot, 1971, AIChE J. 17, 1166.

Lum, K., D. Chandler, and J. D. Weeks, 1999, J. Phys. Chem. B 103, 4570 .

Lumma, D., A. Best, A. Gansen, F. Feuillebois, J. O. Radler, and O. I. Vinogradova, 2003, Phys. Rev. E 67, 056313.

Luo, Q. Z., S. Mutlu, Y. B. Gianchandani, F. Svec, and J. M. J. Frechet, 2003, Electrophoresis 24, 3694.

Lutz, B. R., J. Chen, and D. T. Schwartz, 2003, Proc. Natl. Acad. Sci. U.S.A. 100, 4395.

Lyklema, J., 1991, Fundamentals of Interface and Colloid Science, Vol. 2 (Academic, London).

Lyne, W. H., 1971, J. Fluid Mech. 45, 13.

MacDonald, J. R., 1970, Trans. Faraday Soc. 66, 943.

MacDonald, M. P., G. C. Spalding, and K. Dholakia, 2003, Nature (London) 426, 421.

Mach, P., T. Krupenkin, S. Yang, and J. A. Rogers, 2002, Appl. Phys. Lett. 81, 202.

Magnasco, M. O., 1993, Phys. Rev. Lett. 71, 1477.

Makohliso, S. A., L. Giovangrandi, D. Leonard, H. J. Mathieu, M. Ilegems, and P. Aebischer, 1998, Biosens. Bioelectron. 13, 1227.

Manneville, S., P. Cluzel, J. L. Viovy, D. Chatenay, and F. Caron, 1996, Europhys. Lett. 36, 413.

Manz, A., D. J. Harrison, E. Verpoorte, and H. M. Widmer, 1993, Adv. Chromatogr. 33, 1.
Marko, J. F., and E. D. Siggia, 1995, Macromolecules 28, 8759. Marmottant, P., and S. Hilgenfeldt, 2003, Nature (London) 423, 153.

Marmottant, P., and S. Hilgenfeldt, 2004, Proc. Natl. Acad. Sci. U.S.A. 101, 9523.

Maroja, A. M., F. A. Oliveira, M. Ciesla, and L. Longa, 2001, Phys. Rev. E 63, 061801.

Matsuo, E. S., and T. Tanaka, 1988, J. Chem. Phys. 89, 1695.

Maurer, J., P. Tabeling, P. Joseph, and H. Willaime, 2003, Phys. Fluids 15, 2613.

Maxwell, J., 1879, Philos. Trans. R. Soc. London 170, 231.

Mazouchi, A., and G. M. Homsy, 2000, Phys. Fluids 12, 542.

Mazouchi, A., and G. M. Homsy, 2001, Phys. Fluids 13, 1594.

McConalogue, D. J., and R. S. Srivastava, 1968, Proc. R. Soc. London, Ser. A 307, 37.

McDonald, J. C., D. C. Duffy, J. R. Anderson, D. T. Chiu, H. K. Wu, O. J. A. Schueller, and G. M. Whitesides, 2000, Electrophoresis 21, 27.

McKinley, G. H., R. C. Armstrong, and R. A. Brown, 1993, Philos. Trans. R. Soc. London, Ser. A 344, 265.

McKinley, G. H., J. A. Byars, R. A. Brown, and R. C. Armstrong, 1991, J. Non-Newtonian Fluid Mech. 40, 201.

McKinley, G. H., A. Oztekin, J. A. Byars, and R. A. Brown, 1995, J. Fluid Mech. 285, 123.

McKinley, G. H., P. Pakdel, and A. Oztekin, 1996, J. NonNewtonian Fluid Mech. 67, 19.

McPherson, A., 1999, Crystallization of Biological Macromolecules (Cold Spring Harbor Laboratory Press, Plainview, NY).

McPherson, A., A. J. Malkin, Y. G. Kuznetsov, S. Koszelak, M. Wells, G. Jenkins, J. Howard, and G. Lawson, 1999, J. Cryst. Growth 196, 572.

Meiners, J. C., and S. R. Quake, 2000, Phys. Rev. Lett. 84, 5014.

Melcher, J. R., and W. J. Schwarz, 1968, Phys. Fluids 11, 2604. Melcher, J. R., and G. I. Taylor, 1969, Annu. Rev. Fluid Mech. 1, 111.

Meldrum, D. R., and M. R. Holl, 2002, Science 297, 1197.

Mensing, G. A., T. M. Pearce, M. D. Graham, and D. J. Beebe, 2004, Philos. Trans. R. Soc. London, Ser. A 362, 1059.

Miyata, T., N. Asami, and T. Uragami, 1999, Nature (London) 399, 766.

Miyauchi, M., N. Kieda, S. Hishita, T. Mitsuhashi, A. Nakajima, T. Watanabe, and K. Hashimoto, 2002, Surf. Sci. 511, 401.

Molho, J. I., A. E. Herr, B. P. Mosier, J. G. Santiago, T. W. Kenny, R. A. Brennen, G. B. Gordon, and B. Mohammadi, 2001, Anal. Chem. 73, 1350.

Moon, H., S. K. Cho, R. L. Garrell, and C. J. Kim, 2002, J. Appl. Phys. 92, 4080.

Moorthy, J., and D. J. Beebe, 2002, Lab Chip 2, 76.

Moorthy, J., and D. J. Beebe, 2003, Anal. Chem. 75, 292 A.

Moorthy, J., C. Khoury, J. S. Moore, and D. J. Beebe, 2001, Sens. Actuators B 75, 223.

Morrison, F. A., 1970, J. Colloid Interface Sci. 34, 210.

Morrison, I. D., 1993, Colloids Surf., A 71, 1.

Mpholo, M., C. G. Smith, and A. B. D. Brown, 2003, Sens. Actuators B 92, 262.

Mugele, F., and S. Herminghaus, 2002, Appl. Phys. Lett. 81, 2303.

Muller, S. J., R. G. Larson, and E. S. G. Shaqfeh, 1989, Rheol. Acta 28, 499.

Murtsovkin, V. A., 1996, Colloid J. 58, 341. 
Murtsovkin, V. A., and G. I. Mantrov, 1991, Colloid J. USSR 53, 240.

Nadal, F., F. Argoul, P. Hanusse, B. Pouligny, and A. Ajdari, 2002, Phys. Rev. E 65, 061409.

Nadal, F., F. Argoul, P. Kestener, B. Pouligny, C. Ybert, and A. Ajdari, 2002, Eur. Phys. J. E 9, 387.

Neto, C., V. S. J. Craig, and D. R. M. Williams, 2003, Eur. Phys. J. E 12, S71.

Newman, J. S., 1991, Electrochemical Systems (Prentice-Hall, Englewood Cliffs, NJ).

Ng, J. D., J. A. Gavira, and J. M. Garcia-Ruiz, 2003, J. Struct. Biol. 142, 218.

Ng, J. M. K., I. Gitlin, A. D. Stroock, and G. M. Whitesides, 2002, Electrophoresis 23, 3461.

Nguyen, N., X. Huang, and T. K. Chuan, 2002, J. Fluids Eng. 124, 384.

Nyborg, W. L., 1953, J. Acoust. Soc. Am. 25, 68.

Nyborg, W. L., 1998, in Nonlinear Acoustics, edited by M. F. Hamilton and D. T. Blackstock (Academic, San Diego), pp. 207-231.

O'Brien, R. W., and L. R. White, 1978, J. Chem. Soc., Faraday Trans. 2 74, 16071626.

Oddy, M. H., J. G. Santiago, and J. C. Mikkelsen, 2001, Anal. Chem. 73, 5822.

Oh, S. K., M. Nakagawa, and K. Ichimura, 2002, J. Mater. Chem. 12, 2262.

Okkels, F., and P. Tabeling, 2004, Phys. Rev. Lett. 92, 038301. Olagunju, D. O., 1995, ZAMP 46, 946.

Oldroyd, J. G., 1950, Proc. R. Soc. London, Ser. A $200,523$.

Olsson, A., G. Stemme, and E. Stemme, 1999, J. Micromech. Microeng. 9, 34.

Ondarçuhu, T., and M. Veyssie, 1991, J. Phys. II 1, 75.

Osada, Y., and S. B. Rossmurphy, 1993, Sci. Am. 268 (5), 82.

Ottino, J. M., 1989, The Kinematics of Mixing: Stretching, Chaos, and Transport (Cambridge University Press, Cambridge, England).

Ottino, J. M., and S. Wiggins, 2004, Philos. Trans. R. Soc. London, Ser. A 362, 923.

Pabit, S. A., and S. J. Hagen, 2002, Biophys. J. 83, 2872.

Paegel, B. M., L. D. Hutt, P. C. Simpson, and R. A. Mathies, 2000, Anal. Chem. 72, 3030.

Paik, P., V. K. Pamula, M. G. Pollack, and R. B. Fair, 2003, Lab Chip 3, 28.

Pakdel, P., and G. H. McKinley, 1996, Phys. Rev. Lett. 77, 2459.

Patel, P. D., and E. S. G. Shaqfeh, 2003, J. Chem. Phys. 118, 2941.

Pearson, J. R. A., 1976, Annu. Rev. Fluid Mech. 8, 163.

Perkins, T. T., S. R. Quake, D. E. Smith, and S. Chu, 1994, Science 264, 822.

Perkins, T. T., D. E. Smith, and S. Chu, 1997, Science 276, 2016.

Perkins, T. T., D. E. Smith, R. G. Larson, and S. Chu, 1995, Science 268, 83.

Peters, E. C., F. Svec, and J. M. J. Frechet, 1997, Adv. Mater. (Weinheim, Ger.) 9, 630.

Petrie, C. J. S., and M. M. Denn, 1976, AIChE J. 22, 209.

Peykov, V., A. Quinn, and J. Ralston, 2000, Colloid Polym. Sci. 278, 789.

Phan-Thien, N., 1983, J. Non-Newtonian Fluid Mech. 13, 325. Phan-Thien, N., 1985, J. Non-Newtonian Fluid Mech. 17, 37. Phillips, R. J., 2003, J. Fluid Mech. 481, 77.

Pit, R., H. Hervet, and L. Leger, 2000, Phys. Rev. Lett. 85, 980. Pohl, H. A., 1978, Dielectrophoresis: The Behavior of Neutral
Matter in Nonuniform Electric Fields (Cambridge University Press, Cambridge, England).

Pollack, L., M. W. Tate, N. C. Darnton, J. B. Knight, S. M. Gruner, W. A. Eaton, and R. H. Austin, 1999, Proc. Natl. Acad. Sci. U.S.A. 96, 10115.

Pollack, L., M. W. Tate, A. C. Finnefrock, C. Kalidas, S. Trotter, N. C. Darnton, L. Lurio, R. H. Austin, C. A. Batt, S. M. Gruner, and S. G. J. Mochrie, 2001, Phys. Rev. Lett. 86, 4962.

Pollack, M. G., R. B. Fair, and A. D. Shenderov, 2000, Appl. Phys. Lett. 77, 1725.

Pollack, M. G., A. D. Shenderov, and R. B. Fair, 2002, Lab Chip 2, 96.

Pozrikidis, C., 1992, Boundary Integral and Singularity Methods for Linearized Viscous Flow (Cambridge University Press, Cambridge, England).

Prins, M. W. J., W. J. J. Welters, and J. W. Weekamp, 2001, Science 291, 277.

Probstein, R. F., 1994, Physicochemical Hydrodynamics (Wiley, New York).

Prost, J., J. F. Chauwin, L. Peliti, and A. Ajdari, 1994, Phys. Rev. Lett. 72, 2652.

Purcell, E. M., 1977, Am. J. Phys. 45, 3.

Qian, S. Z., and H. H. Bau, 2002, Anal. Chem. 74, 3616.

Qiao, R., and N. R. Aluru, 2003, Sens. Actuators, A 104, 268.

Quake, S. R., 1994, J. Chem. Phys. 101, 4307.

Quake, S. R., and A. Scherer, 2000, Science 290, 1536.

Quilliet, C., and B. Berge, 2001, Curr. Opin. Colloid Interface Sci. 6, 34.

Rabai, G., M. Orban, and I. R. Epstein, 1990, Acc. Chem. Res. 23, 258.

Rallison, J. M., 1984, Annu. Rev. Fluid Mech. 16, 45.

Rallison, J. M., and E. J. Hinch, 1988, J. Non-Newtonian Fluid Mech. 29, 37.

Ramos, A., A. Gonzalez, A. Castellanos, N. G. Green, and H. Morgan, 2003, Phys. Rev. E 67, 056302.

Ramos, A., H. Morgan, N. G. Green, and A. Castellanos, 1999, J. Colloid Interface Sci. 217, 420.

Ramsey, J. M., 1999, Nat. Biotechnol. 17, 1061.

Ramsey, J. M., S. C. Jacobson, and M. R. Knapp, 1995, Nat. Med. (N.Y.) 1, 1093.

Raphael, E., 1988, C. R. Acad. Sci., Ser. IIb: Mec., Phys., Chim., Astron. 306, 751.

Raviv, U., P. Laurat, and J. Klein, 2001, Nature (London) 413, 51.

Rayleigh, L., 1879, Proc. London Math. Soc. 10, 4.

Rayleigh, L., 1884, Philos. Trans. R. Soc. London 175, 1.

Reiner, M., 1964, Phys. Today 17 (1), 62.

Ren, H., R. B. Fair, and M. G. Pollack, 2004, Sens. Actuators B 98, 319.

Ren, L. Q., C. Escobedo, and D. Li, 2001, J. Colloid Interface Sci. 242, 264.

Ren, L. Q., and D. Q. Li, 2001, J. Colloid Interface Sci. 243, 255.

Reyes, D. R., D. Iossifidis, P. A. Auroux, and A. Manz, 2002, Anal. Chem. 74, 2623.

Richardson, S., 1973, J. Fluid Mech. 59, 707.

Richter, A., D. Kuckling, S. Howitz, T. Gehring, and K. F. Arndt, 2003, J. Microelectromech. Syst. 12, 748.

Rife, J. C., M. I. Bell, J. S. Horwitz, M. N. Kabler, R. C. Y. Aeuyeung, and W. J. Kim, 2000, Sens. Actuators, A 86, 135. Riley, N., 2001, Annu. Rev. Fluid Mech. 33, 43.

Ristenpart, W. D., I. A. Aksay, and D. A. Saville, 2003, Phys. Rev. Lett. 90, 128303. 
Rodd, L. E., T. P. Scott, D. V. Boger, J. J. Cooper-White, and G. H. McKinley, 2004, XIVth International Congress on Rheology (The Korean Society of Rheology, Seoul, South Korea). Roques-Carmes, T., R. A. Hayes, B. J. Feenstra, and L. J. M. Schlangen, 2004, J. Appl. Phys. 95, 4389.

Rosslee, C. A., and N. L. Abbott, 2001, Anal. Chem. 73, 4808.

Rothstein, J. P., and G. H. McKinley, 2001, Phys. Fluids 13, 382.

Roy, I., and M. N. Gupta, 2003, Chem. Biol. 10, 1161.

Ruckenstein, E., and N. Churaev, 1991, J. Colloid Interface Sci. 147, 535.

Ruckenstein, E., and P. Rajora, 1983, J. Colloid Interface Sci. 96, 488.

Rush, B. M., K. D. Dorfman, H. Brenner, and S. Kim, 2002, Ind. Eng. Chem. Res. 41, 4652.

Russel, W. B., D. A. Saville, and W. R. Schowalter, 1989, Colloidal Dispersions (Cambridge University Press, Cambridge, England).

Saeki, F., J. Baum, H. Moon, J. Y. Yoon, C. J. Kim, and R. L. Garrell, 2001, Polym. Mater. Sci. Eng. 85, 12.

Sakai, T., and R. Yoshida, 2004, Langmuir 20, 1036.

Salemme, F. R., 1972, Arch. Biochem. Biophys. 151, 533.

Sammarco, T. S., and M. A. Burns, 1999, AIChE J. 45, 350.

Sammarco, T. S., and M. A. Burns, 2000, J. Micromech. Microeng. 10, 42.

Sartori, A., V. Barbier, and J. L. Viovy, 2003, Electrophoresis 24, 421.

Saville, D. A., 1977, Annu. Rev. Fluid Mech. 9, 321.

Saville, D. A., 1997, Annu. Rev. Fluid Mech. 29, 27.

Sawano, A., S. Takayama, M. Matsuda, and A. Miyawaki, 2002, Dev. Cell 3, 245.

Schasfoort, R. B. M., S. Schlautmann, L. Hendrikse, and A. van den Berg, 1999, Science 286, 942.

Schilling, E. A., A. E. Kamholz, and P. Yager, 2002, Anal. Chem. 74, 1798.

Schmalzing, D., L. Koutny, A. Adourian, P. Belgrader, P. Matsudaira, and D. Ehrlich, 1997, Proc. Natl. Acad. Sci. U.S.A 94, 10273.

Schneemilch, M., W. J. J. Welters, R. A. Hayes, and J. Ralston, 2000, Langmuir 16, 2924.

Schnell, E., 1956, J. Appl. Phys. 27, 1149.

Schroeder, C. M., H. P. Babcock, E. S. G. Shaqfeh, and S. Chu, 2003, Science 301, 1515.

Schwartz, J. A., J. V. Vykoukal, and P. R. C. Gascoyne, 2004, Lab Chip 4, 11.

Selvaganapathy, P., E. T. Carlen, and C. H. Mastrangelo, 2003, Sens. Actuators, A 104, 275.

Seong, G. H., and R. M. Crooks, 2002, J. Am. Chem. Soc. 124, 13360.

Seyrat, E., and R. A. Hayes, 2001, J. Appl. Phys. 90, 1383.

Shanahan, M. E. R., and P. G. de Gennes, 1997, C. R. Acad. Sci., Ser. IIb: Mec., Phys., Chim., Astron. 324, 261.

Shaqfeh, E. S. G., 1996, Annu. Rev. Fluid Mech. 28, 129.

Shaqfeh, E. S. G., S. J. Muller, and R. G. Larson, 1992, J. Fluid Mech. 235, 285.

Sharp, M. K., R. D. Kamm, A. H. Shapiro, E. Kimmel, and G. E. Karniadakis, 1991, J. Fluid Mech. 223, 537.

Shin, J. Y., and N. L. Abbott, 1999, Langmuir 15, 4404.

Sia, S. K., and G. M. Whitesides, 2003, Electrophoresis 24, 3563.

Simonov, I. N., and V. N. Shilov, 1977, Colloid J. USSR 39, 775.

Slater, G. W., S. Guillouzic, M. G. Gauthier, J. F. Mercier, M.
Kenward, L. C. McCormick, and F. Tessier, 2002, Electrophoresis 23, 3791.

Slater, G. W., M. Kenward, L. C. McCormick, and M. G. Gauthier, 2003, Curr. Opin. Biotechnol. 14, 58.

Smith, D. E., H. P. Babcock, and S. Chu, 1999, Science 283, 1724.

Smith, D. E., and S. Chu, 1998, Science 281, 1335.

Smith, S. B., L. Finzi, and C. Bustamante, 1992, Science 258, 1122.

Solomentsev, Y., and J. L. Anderson, 1995, Ind. Eng. Chem. Res. 34, 3231.

Solomon, T. H., and I. Mezic, 2003, Nature (London) 425, 376.

Sondag-Huethorst, J. A. M., and L. G. J. Fokkink, 1994, Langmuir 10, 4380.

Song, H., M. R. Bringer, J. D. Tice, C. J. Gerdts, and R. F. Ismagilov, 2003, Appl. Phys. Lett. 83, 4664.

Song, H., and R. F. Ismagilov, 2003, J. Am. Chem. Soc. 125, 14613.

Song, H., J. D. Tice, and R. F. Ismagilov, 2003, Angew. Chem., Int. Ed. 42, 768.

Spikes, H., and S. Granick, 2003, Langmuir 19, 5065.

Squires, T. M., and M. Z. Bazant, 2004, J. Fluid Mech. 509, 217. Stemme, E., and G. Stemme, 1993, Sens. Actuators, A 39, 159. Stockle, R., C. Fokas, V. Deckert, R. Zenobi, B. Sick, B. Hecht, and U. Wild, 1999, Appl. Phys. Lett. 75, 160.

Stone, H. A., 1994, Annu. Rev. Fluid Mech. 26, 65.

Stone, H. A., and S. Kim, 2001, AIChE J. 47, 1250.

Stone, H. A., A. Nadim, and S. H. Strogatz, 1991, J. Fluid Mech. 232, 629.

Stone, H. A., A. D. Stroock, and A. Ajdari, 2004, Annu. Rev. Fluid Mech. 36, 381411.

Stremler, M. A., F. R. Haselton, and H. Aref, 2004, Philos. Trans. R. Soc. London, Ser. A 362, 1019.

Stroock, A. D., S. K. Dertinger, G. M. Whitesides, and A. Ajdari, 2002, Anal. Chem. 74, 5306.

Stroock, A. D., S. K. W. Dertinger, A. Ajdari, I. Mezic, H. A. Stone, and G. M. Whitesides, 2002, Science 295, 647.

Stroock, A. D., R. F. Ismagilov, H. A. Stone, and G. M. Whitesides, 2003, Langmuir 19, 4358.

Stroock, A. D., and G. J. McGraw, 2004, Philos. Trans. R. Soc. London, Ser. A 362, 971.

Stroock, A. D., M. Weck, D. T. Chiu, W. T. S. Huck, P. J. A. Kenis, R. F. Ismagilov, and G. M. Whitesides, 2000, Phys. Rev. Lett. 84, 3314.

Stuart, J. T., 1963, in Laminar Boundary Layers, edited by L. Rosenhead (Clarendon, Oxford), pp. 349-408.

Stuart, J. T., 1966, J. Fluid Mech. 24, 673.

Studer, V., G. Hang, A. Pandolfi, M. Ortiz, W. F. Anderson, and S. R. Quake, 2004, J. Appl. Phys. 95, 393.

Studer, V., A. Pepin, Y. Chen, and A. Ajdari, 2002, Microelectron. Eng. 61-2, 915.

Sugiura, S., M. Nakajima, S. Iwamoto, and M. Seki, 2001, Langmuir 17, 5562.

Sugiura, S., M. Nakajima, and M. Seki, 2002a, Langmuir 18, 5708.

Sugiura, S., M. Nakajima, and M. Seki, 2002b, Langmuir 18, 3854.

Sugiura, S., M. Nakajima, J. H. Tong, H. Nabetani, and M. Seki, 2000, J. Colloid Interface Sci. 227, 95.

Sun, G. X., E. Bonaccurso, V. Franz, and H. J. Butt, 2002, J. Chem. Phys. 117, 10311.

Suzuki, A., and T. Tanaka, 1990, Nature (London) 346, 345.

Tabeling, P., M. Chabert, A. Dodge, C. Jullien, and F. Okkels, 
2004, Philos. Trans. R. Soc. London, Ser. A 362, 987.

Takayama, S., J. C. McDonald, E. Ostuni, M. N. Liang, P. J. A. Kenis, R. F. Ismagilov, and G. M. Whitesides, 1999, Proc. Natl. Acad. Sci. U.S.A. 96, 5545.

Takayama, S., E. Ostuni, P. LeDuc, K. Naruse, D. E. Ingber, and G. M. Whitesides, 2001, Nature (London) 411, 1016.

Takayama, S., E. Ostuni, P. LeDuc, K. Naruse, D. E. Ingber, and G. M. Whitesides, 2003, Chem. Biol. 10, 123.

Takhistov, P., K. Duginova, and H. C. Chang, 2003, J. Colloid Interface Sci. 263, 133.

Tanaka, T., 1978, Phys. Rev. Lett. 40, 820.

Tanaka, T., D. Fillmore, S. T. Sun, I. Nishio, G. Swislow, and A. Shah, 1980, Phys. Rev. Lett. 45, 1636.

Tanaka, T., I. Nishio, S. T. Sun, and S. Uenonishio, 1982, Science 218, 467.

Taniguchi, T., T. Torii, and T. Higuchi, 2002, Lab Chip 2, 19.

Tatsuno, M., 1980, Rep. Res. Inst. Appl. Mech. (Kyushu Univ.) 28, 35 .

Taylor, G., 1953, Proc. R. Soc. London, Ser. A 219, 186.

Taylor, G., 1954, Proc. R. Soc. London, Ser. A 225, 473.

Taylor, G. I., 1934, Proc. R. Soc. London, Ser. A 146, 501.

Taylor, G. I., 1966, Proc. R. Soc. London, Ser. A 291, 159.

Tegenfeldt, J. O., C. Prinz, H. Cao, R. L. Huang, R. H. Austin, S. Y. Chou, E. C. Cox, and J. C. Sturm, 2004, Anal. Bioanal. Chem. 378, 1678.

Teubner, M., 1982, J. Chem. Phys. 76, 5564.

Thamida, S. K., and H. C. Chang, 2002, Phys. Fluids 14, 4315. Thompson, P. A., and M. O. Robbins, 1990, Phys. Rev. A 41, 6830 .

Thompson, P. A., and S. M. Troian, 1997, Nature (London) 389, 360.

Thormann, W., I. S. Lurie, B. McCord, U. Marti, B. Cenni, and N. Malik, 2001, Electrophoresis 22, 4216.

Thorsen, T., S. J. Maerkl, and S. R. Quake, 2002, Science 298, 580.

Thorsen, T., R. W. Roberts, F. H. Arnold, and S. R. Quake, 2001, Phys. Rev. Lett. 86, 4163.

Tice, J. D., H. Song, A. D. Lyon, and R. F. Ismagilov, 2003, Langmuir 19, 9127.

Torza, S., R. G. Cox, and S. G. Mason, 1971, Philos. Trans. R. Soc. London, Ser. A 269, 295.

Trau, M., D. A. Saville, and I. A. Aksay, 1997, Langmuir 13, 6375 .

Tretheway, D. C., and C. D. Meinhart, 2002, Phys. Fluids 14, L9.

Tretheway, D. C., and C. D. Meinhart, 2004, Phys. Fluids 16, 1509.

Turner, S. W. P., M. Cabodi, and H. G. Craighead, 2002, Phys. Rev. Lett. 88, 128103.

Tyrrell, J. W. G., and P. Attard, 2001, Phys. Rev. Lett. 87, 176104.

Tyrrell, J. W. G., and P. Attard, 2002, Langmuir 18, 160.

Ugaz, V. M., R. D. Elms, R. C. Lo, F. A. Shaikh, and M. A. Burns, 2004, Philos. Trans. R. Soc. London, Ser. A 362, 1105. Unger, M. A., H. P. Chou, T. Thorsen, A. Scherer, and S. R. Quake, 2000, Science 288, 113.

Unger, M. A., D. A. Kossakovski, R. Kongovi, J. L. Beauchamp, J. D. Baldeschwieler, and D. V. Palanker, 1998, Rev. Sci. Instrum. 69, 2988.

Vallet, M., M. Vallade, and B. Berge, 1999, Eur. Phys. J. B 11, 583.

van der Vegt, W., W. Norde, H. C. van der Mei, and H. J. Busscher, 1996, J. Colloid Interface Sci. 179, 57.
Vekilov, P. G., B. R. Thomas, and F. Rosenberger, 1998, J. Phys. Chem. B 102, 5208.

Verheijen, H. J. J., and M. W. J. Prins, 1999, Langmuir 15, 6616.

Verpoorte, E., 2002, Electrophoresis 23, 677.

Verpoorte, E., and N. F. De Rooij, 2003, Proc. IEEE 91, 930.

Vinogradova, O. I., 1995, Langmuir 11, 2213.

Vinogradova, O. I., 1996, Langmuir 12, 5963.

Vinogradova, O. I., 1999, Int. J. Min. Process. 56, 31.

Vinogradova, O. I., and G. E. Yakubov, 2003, Langmuir 19, 1227.

Viovy, J. L., 2000, Rev. Mod. Phys. 72, 813.

Volkmuth, W. D., and R. H. Austin, 1992, Nature (London) 358, 600 .

Volkmuth, W. D., T. Duke, M. C. Wu, R. H. Austin, and A. Szabo, 1994, Phys. Rev. Lett. 72, 2117.

Vologodskii, A., 1994, Macromolecules 27, 5623.

Walker, G. M., M. S. Ozers, and D. J. Beebe, 2002, Biomed. Microdevices 4, 161.

Wang, R., K. Hashimoto, A. Fujishima, M. Chikuni, E. Kojima, A. Kitamura, M. Shimohigoshi, and T. Watanabe, 1997, Nature (London) 388, 431.

Ward, T., and G. M. Homsy, 2001, Phys. Fluids 13, 3521.

Ward, T., and G. M. Homsy, 2003, Phys. Fluids 15, 2987.

Wasan, D. T., A. D. Nikolov, and H. Brenner, 2001, Science 291, 605.

Washburn, E. W., 1921, Phys. Rev. 17, 273.

Washizu, M., 1998, IEEE Trans. Ind. Appl. 34, 732.

Watanabe, K., Yanuar, and H. Udagawa, 1999, J. Fluid Mech. 381, 225.

Weigl, B. H., and P. Yager, 1999, Science 283, 346.

Weislogel, M. M., 1997, AIChE J. 43, 645.

Welters, W. J. J., and L. G. J. Fokkink, 1998, Langmuir 14, 1535 .

Westervelt, P. J., 1953, J. Acoust. Soc. Am. 25, 60.

Wheeler, A. R., W. R. Throndset, R. J. Whelan, A. M. Leach, R. N. Zare, Y. H. Liao, K. Farrell, I. D. Manger, and A. Daridon, 2003, Anal. Chem. 75, 3581.

White, J. M., and S. J. Muller, 2000, Phys. Rev. Lett. 84, 5130.

White, J. M., and S. J. Muller, 2003, J. Rheol. 47, 1467.

Whitesides, G. M., E. Ostuni, S. Takayama, X. Jiang, and D. E. Ingber, 2001, Annu. Rev. Biomed. Eng. 3, 335.

Whitesides, G. M., and A. D. Stroock, 2001, Phys. Today 54 (6), 42.

Wiggins, S., and J. M. Ottino, 2004, Philos. Trans. R. Soc. London, Ser. A 362, 937.

Wilbur, J. L., A. Kumar, E. Kim, and G. M. Whitesides, 1994, Adv. Mater. (Weinheim, Ger.) 6, 600.

Wilding, P., M. A. Shoffner, and L. J. Kricka, 1994, Clin. Chem. 40, 1815.

Wong, P. K., Y.-K. Lee, and C.-M. Ho, 2003, J. Fluid Mech. 495, 55.

Woo, N. J., E. S. G. Shaqfeh, and B. Khomami, 2004a, J. Rheol. 48, 299.

Woo, N. J., E. S. G. Shaqfeh, and B. Khomami, 2004b, J. Rheol. 48, 281.

Woolley, A. T., D. Hadley, P. Landre, A. J. deMello, R. A. Mathies, and M. A. Northrup, 1996, Anal. Chem. 68, 4081.

Xia, Y. N., and G. M. Whitesides, 1998, Annu. Rev. Mater. Sci. 28, 153.

Yang, H., and R. L. Chien, 2001, J. Chromatogr., A 924, 155.

Yang, S., T. N. Krupenkin, P. Mach, and E. A. Chandross, 2003, Adv. Mater. (Weinheim, Ger.) 15, 940. 
Yang, Z., H. Goto, M. Matsumoto, and R. Maeda, 2000, Electrophoresis 21, 116.

Yang, Z., S. Matsumoto, H. Goto, M. Matsumoto, and R. Maeda, 2001, Sens. Actuators, A 93, 266.

Yeh, S. R., M. Seul, and B. I. Shraiman, 1997, Nature (London) 386, 57.

Yi, G. R., S. J. Jeon, T. Thorsen, V. N. Manoharan, S. R. Quake, D. J. Pine, and S. M. Yang, 2003, Synth. Met. 139, 803.

Yi, G. R., T. Thorsen, V. N. Manoharan, M. J. Hwang, S. J. Jeon, D. J. Pine, S. R. Quake, and S. M. Yang, 2003, Adv. Mater. (Weinheim, Ger.) 15, 1300.

Yoon, J. Y., and R. L. Garrell, 2003, Anal. Chem. 75, 5097.

Yoshida, R., T. Takahashi, T. Yamaguchi, and H. Ichijo, 1996, J. Am. Chem. Soc. 118, 5134.

Yoshida, R., K. Takei, and T. Yamaguchi, 2003, Macromolecules 36, 1759.

Young, N. O., J. S. Goldstein, and M. J. Block, 1959, J. Fluid Mech. 6, 350.

Yu, C., S. Mutlu, P. Selvaganapathy, C. H. Mastrangelo, F. Svec, and J. M. J. Frechett, 2003, Anal. Chem. 75, 1958.

Yu, Q., J. M. Bauer, J. S. Moore, and D. J. Beebe, 2001, Appl. Phys. Lett. 78, 2589.

Yun, K. S., I. J. Cho, J. U. Bu, C. J. Kim, and E. Yoon, 2002, J.
Microelectromech. Syst. 11, 454.

Zhan, W., G. H. Seong, and R. M. Crooks, 2002, Anal. Chem. 74, 4647.

Zhang, L., J. M. Koo, L. Jiang, M. Asheghi, K. E. Goodson, J. G. Santiago, and T. W. Kenny, 2002, J. Microelectromech. Syst. 11, 12.

Zhao, B., J. S. Moore, and D. J. Beebe, 2001, Science 291, 1023.

Zhao, B., J. S. Moore, and D. J. Beebe, 2002, Anal. Chem. 74, 4259 .

Zhao, B., J. S. Moore, and D. J. Beebo, 2003, Langmuir 19, 1873.

Zhao, B., N. O. L. Viernes, J. S. Moore, and D. J. Beebe, 2002, J. Am. Chem. Soc. 124, 5284.

Zheng, B., L. S. Roach, and R. F. Ismagilov, 2003, J. Am. Chem. Soc. 125, 11170.

Zhu, X., and E. S. Kim, 1998, Sens. Actuators, A 66, 355.

Zhu, Y. X., and S. Granick, 2001, Phys. Rev. Lett. 87, 096105.

Zhu, Y. X., and S. Granick, 2002a, Phys. Rev. Lett. 88, 106102. Zhu, Y. X., and S. Granick, 2002b, Langmuir 18, 10058.

Zubritsky, E., 2000, Anal. Chem. 72, 687A.

Zwieniecki, M. A., P. J. Melcher, and N. M. Holbrook, 2001, Science 291, 1059. 